\title{
HILBERT'S 6TH PROBLEM: \\ EXACT AND APPROXIMATE HYDRODYNAMIC MANIFOLDS FOR KINETIC EQUATIONS
}

\author{
ALEXANDER N. GORBAN AND ILYA KARLIN
}

\begin{abstract}
The problem of the derivation of hydrodynamics from the Boltzmann equation and related dissipative systems is formulated as the problem of a slow invariant manifold in the space of distributions. We review a few instances where such hydrodynamic manifolds were found analytically both as the result of summation of the Chapman-Enskog asymptotic expansion and by the direct solution of the invariance equation. These model cases, comprising Grad's moment systems, both linear and nonlinear, are studied in depth in order to gain understanding of what can be expected for the Boltzmann equation. Particularly, the dispersive dominance and saturation of dissipation rate of the exact hydrodynamics in the short-wave limit and the viscosity modification at high divergence of the flow velocity are indicated as severe obstacles to the resolution of Hilbert's 6th Problem. Furthermore, we review the derivation of the approximate hydrodynamic manifold for the Boltzmann equation using Newton's iteration and avoiding smallness parameters, and compare this to the exact solutions. Additionally, we discuss the problem of projection of the Boltzmann equation onto the approximate hydrodynamic invariant manifold using entropy concepts. Finally, a set of hypotheses is put forward where we describe open questions and set a horizon for what can be derived exactly or proven about the hydrodynamic manifolds for the Boltzmann equation in the future.
\end{abstract}

\section{Contents}

1. Introduction

1.1. Hilbert's 6th Problem

1.2. The main equations

1.3. Singular perturbation and separation of times in kinetics 192

1.4. The structure of this paper

2. Invariance equation and the Chapman-Enskog expansion 196

2.1. The idea of an invariant manifold in kinetics 196

2.2. The Chapman-Enskog expansion 199

2.3. Euler, Navier-Stokes, Burnett, and super-Burnett terms for a simple kinetic equation

3. Algebraic hydrodynamic invariant manifolds and exact summation of the Chapman-Enskog series for the simplest kinetic model

3.1. Grin of the vanishing cat: $\epsilon=1$

3.2. The pseudodifferential form of the stress tensor

Received by the editors August 28, 2013, and, in revised form, September 25, 2013. 2010 Mathematics Subject Classification. Primary 76P05, 82B40, 35 Q35. 
3.3. The energy formula and "capillarity" of ideal gas

3.4. Algebraic invariant manifold in Fourier representation

3.5. Stability of the exact hydrodynamic system and saturation of dissipation for short waves

3.6. Expansion at $k^{2}=\infty$ and matched asymptotics

4. Algebraic invariant manifold for general linear kinetics in one dimension 211

4.1. General form of the invariance equation for one-dimensional linear kinetics

4.2. Hyperbolicity of exact hydrodynamics

4.3. Destruction of hydrodynamic invariant manifold for short waves in moment equations

4.4. Invariant manifolds, entanglement of hydrodynamic and nonhydrodynamic modes, and saturation of dissipation for the three-dimensional 13-moment Grad system

4.5. Algebraic hydrodynamic invariant manifold for the linearized Boltzmann and BGK equations: separation of hydrodynamic and nonhydrodynamic modes

5. Hydrodynamic invariant manifolds for nonlinear kinetics

5.1. One-dimensional nonlinear Grad equation and nonlinear viscosity

5.2. Approximate invariant manifold for the Boltzmann equation

6. The projection problem and the entropy equation

7. Conclusion

Acknowledgments

References

\section{INTRODUCTION}

1.1. Hilbert's 6th Problem. The 6th Problem differs significantly from the other 22 Hilbert problems [76]. The title of the problem itself is mysterious: "Mathematical treatment of the axioms of physics". Physics, in its essence, is a special activity for the creation, validation, and destruction of theories for real-world phenomena, where "We are trying to prove ourselves wrong as quickly as possible, because only in that way can we find progress" [38. There exist no mathematical tools to formalize relations between theory and reality in live physics. Therefore, the 6th Problem may be viewed as a tremendous challenge in the deep study of ideas of physical reality in order to replace vague philosophy by a new logical and mathematical discipline. Some research in quantum observation theory and related topics can be viewed as steps in that direction, but it seems that, at present, we are far from an understanding of the most logical and mathematical problems here.

The first explanation of the 6th Problem given by Hilbert reduced the level of challenge and made the problem more tractable: "The investigations on the foundations of geometry suggest the problem: To treat in the same manner, by means of axioms, those physical sciences in which mathematics plays an important part; in the first rank are the theory of probabilities and mechanics." This is definitely "a programmatic call" 23] for the axiomatization of the formal parts of existent physical theories and no new universal logical framework for the representation of 
reality is necessary. In this context, the axiomatic approach is a tool for the retrospective analysis of well-established and elaborated physical theories $[23$ and not for live physics.

For the general statements of the 6th Problem it seems unclear now how to formulate criteria of solutions. In a further explanation Hilbert proposed two specific problems: (i) axiomatic treatment of probability with limit theorems for the foundation of statistical physics, and (ii) the rigorous theory of limiting processes "which lead from the atomistic view to the laws of motion of continua". For complete resolution of these problems, Hilbert has set no criteria either, but some important parts of them have already been claimed as solved. Several axiomatic approaches to probability have been developed, and the equivalence of some of them has been proven [45. Kolmogorov's axiomatics (1933) 96] is now accepted as standard, and thirty years later, the complexity approach to randomness was invented by Solomonoff and Kolmogorov (see the review [148] and the textbook [107]). The rigorous foundation of equilibrium statistical physics of many particles based on the central limit theorems was proposed [30,95]. The modern development of limit theorems in high dimensions is based on the geometrical ideas of measure concentration effects [72,137], and this gives new insight into the foundation of statistical physics (see, for example, [47,138]). Despite many open questions, this part of the Hilbert program is essentially fulfilled - probability theory and the foundations of equilibrium statistical physics are now well-established chapters of mathematics.

The way from the "atomistic view to the laws of motion of continua" is not so well formalized. It includes at least two steps: (i) from mechanics to kinetics (from Newton to Boltzmann), and (ii) from kinetics to mechanics and nonequilibrium thermodynamics of continua (from Boltzmann to Euler and Navier, Stokes, and Fourier).

The first part of the problem, the transition from the reversible-in-time equations of mechanics to irreversible kinetic equations, is still far from being a complete rigorous theory. The highest achievement here is the proof that rarefied gas of hard spheres will follow the Boltzmann equation during a fraction of the collision time, starting from a noncorrelated initial state [43, 104. The Bogoliubov-BornGreen-Kirkwood-Yvon (BBGKY) hierarchy [13] provides the general framework for this problem. For the systems close to global thermodynamic equilibrium, the global-in-time estimates are available, and the validity of the linearized Boltzmann equation is proven recently in this limit for rarefied gas of hard spheres [12.

The second part, model reduction in dissipative systems from kinetics to macroscopic dynamics, is ready for a mathematical treatment. Some limit theorems about this model reduction are already proven (see the review book [126] and the companion paper by L. Saint-Raymond [127] in this volume), and open questions can be presented in a rigorous mathematical form. Our review is focused on this model reduction problem, which is important in many areas of kinetics from the Boltzmann equation to chemical kinetics. There exist many similar heuristic approaches for different applications [60, 112, 124, 129].

It seems that Hilbert presumed the kinetic level of description (the "Boltzmann level") as an intermediate step between the microscopic mechanical description and the continuum mechanics. Nevertheless, this intermediate description may be omitted. The transition from the microscopic to the macroscopic description without an intermediate kinetic equation is used in many physical theories such as 
Green-Kubo formalism [100, the Zubarev method of a nonequilibrium statistical operator [147], and the projection operator techniques [67. This possibility is demonstrated rigorously for a rarefied gas near global equilibrium [12].

The reduction from Boltzmann kinetics to hydrodynamics may be split into three problems: existence of hydrodynamics, the form of the hydrodynamic equations, and the relaxation of the Boltzmann kinetics to hydrodynamics. Formalization of these problems is a crucial step in the analysis.

Three questions arise:

(1) Is there hydrodynamics in the kinetic equation, i.e., is it possible to lift the hydrodynamic fields to the relevant one-particle distribution functions in such a way that the projection of the kinetics of the relevant distributions satisfies some hydrodynamic equations?

(2) Do these hydrodynamics have the conventional Euler and Navier-Stokes Fourier form?

(3) Do the solutions of the kinetic equation degenerate to the hydrodynamic regime (after some transient period)?

The first question is the problem of existence of a hydrodynamic invariant manifold for kinetics (this manifold should be parametrized by hydrodynamic fields). The second question is about the form of hydrodynamic equations obtained by the natural projection of kinetic equations from the invariant manifold. The third question is about the intermediate asymptotics of the relaxation of kinetics to equilibrium: Do the solutions go fast to the hydrodynamic invariant manifold and then follow this manifold on the path to equilibrium?

The answer to all three questions is essentially positive in the asymptotic regime when the Mach number $M a$ and the Knudsen number $K n$ tend to zero [6, 46] (see [126, 127]). This is a limit of very slow flows with very small gradients of all fields, i.e., almost no flow at all. Such a flow changes in time very slowly and a rescaling of time $t_{\text {old }}=t_{\text {new }} / \varepsilon$ is needed to return it to nontrivial dynamics (the so-called diffusive rescaling). After the rescaling, we approach in this limit the Euler and Navier-Stokes Fourier hydrodynamics of incompressible liquids.

Thus in the limit $M a, K n \rightarrow 0$ and after rescaling, Hilbert's 6th Problem is essentially resolved and the result meets Hilbert's expectations: the continuum equations are rigorously derived from the Boltzmann equation. Besides the limit the answers are known partially. To the best of our knowledge, now the answers to these three questions are: (1) sometimes; (2) not always; (3) possibly.

Some hints about the problems with hydrodynamic asymptotics can be found in the series of works about the small dispersion limit of the Korteweg-de Vries equation [105]. Recently, analysis of the exact solution of the model reduction problem for a simple kinetic model [57, 135] has demonstrated that a hydrodynamic invariant manifold may exist and produce nonlocal hydrodynamics. Analysis of more complicated kinetics [19, 20, 86, 87, 90, supports and extends these observations: the hydrodynamic invariant manifold may exist, but sometimes does not exist; and the hydrodynamic equations when $M a \nrightarrow 0$ may differ essentially from the Euler and Navier-Stokes Fourier equations.

At least two effects prevent us from giving positive answers to the first two questions outside of the limit $M a, K n \rightarrow 0$ :

- Entanglement between the hydrodynamic and nonhydrodynamic modes may destroy the hydrodynamic invariant manifold. 
- Saturation of dissipation at high frequencies is a universal effect that does not appear in the classical hydrodynamic equations.

These effects appear already in simple linear kinetic models and are studied in detail for exactly solvable reduction problems. The entanglement between the hydrodynamic and nonhydrodynamic modes manifests itself in many popular moment approximations for the Boltzmann equation. In particular, it exists for the threedimensional 10-moment and 13-moment Grad systems $19,20,60,86,90$, but the numerical study of hydrodynamic invariant manifolds for the Bhatnagar-Gross-Krook (BGK) model equation [87. demonstrates the absence of such an entanglement. Therefore, our conjecture is that for the Boltzmann equation, exact hydrodynamic modes are separated from the nonhydrodynamic ones if the linearized collision operator has a spectral gap between the five-times degenerated zero and the rest of the spectrum.

The saturation of dissipation seems to be a universal phenomenon [52, 53, 60, 90 , 101, 123, 132. It appears in all exactly solved reduction problems for kinetic equations [90] and in BGK kinetics [7,87] and is also proven for various regularizations of the Chapman-Enskog expansion [52,60, 123, 132.

The answer to Hilbert's 6th Problem concerning transition from the Boltzmann equation to the classical equations of motion of compressible continua $(M a \nrightarrow 0)$ may turn out to be negative. Even if we can overcome the first difficulty, separating the hydrodynamic modes from the nonhydrodynamic ones (as in the exact solution [57] or for the BGK equation [87]) and producing the hydrodynamic equations from the Boltzmann equation, the result will be manifestly different from the conventional equations of hydrodynamics.

1.2. The main equations. We discuss here two groups of examples. The first of them consists of kinetic equations which describe the evolution of a one-particle gas distribution function $f(t, \boldsymbol{x} ; \boldsymbol{v})$

$$
\partial_{t} f+\boldsymbol{v} \cdot \nabla_{x} f=\frac{1}{\epsilon} Q(f),
$$

where $Q(f)$ is the collision operator. For the Boltzmann equation, $Q$ is a quadratic operator and, therefore, the notation $Q(f, f)$ is often used.

The second group of examples are the systems of Grad moment equations 9,60 , 68, 84. The system of 13-moment Grad equations linearized near equilibrium is

$$
\begin{aligned}
\partial_{t} \rho & =-\nabla \cdot \boldsymbol{u}, \\
\partial_{t} \boldsymbol{u} & =-\nabla \rho-\nabla T-\nabla \cdot \boldsymbol{\sigma}, \\
\partial_{t} T & =-\frac{2}{3}(\nabla \cdot \boldsymbol{u}+\nabla \cdot \boldsymbol{q}), \\
\partial_{t} \boldsymbol{\sigma} & =-2 \overline{\nabla \boldsymbol{u}}-\frac{4}{5} \overline{\nabla \boldsymbol{q}}-\frac{1}{\epsilon} \boldsymbol{\sigma}, \\
\partial_{t} \boldsymbol{q} & =-\frac{5}{2} \nabla T-\nabla \cdot \boldsymbol{\sigma}-\frac{2}{3 \epsilon} \boldsymbol{q} .
\end{aligned}
$$

In these equations, $\boldsymbol{\sigma}(\boldsymbol{x}, t)$ is the dimensionless stress tensor, $\boldsymbol{\sigma}=\left(\sigma_{i j}\right)$, and $\boldsymbol{q}(\boldsymbol{x}, t)$ is the dimensionless vector of heat flux, $\boldsymbol{q}=\left(q_{i}\right)$. We use the system of units in which Boltzmann's constant $k_{\mathrm{B}}$ and the particle mass $m$ are equal to 1 , and we use 
the system of dimensionless variables,

$$
\boldsymbol{u}=\frac{\delta \boldsymbol{u}}{\sqrt{T_{0}}}, \quad \rho=\frac{\delta \rho}{\rho_{0}}, \quad T=\frac{\delta T}{T_{0}}, \quad \boldsymbol{x}=\frac{\rho_{0}}{\eta\left(T_{0}\right) \sqrt{T_{0}}} \boldsymbol{x}^{\prime}, \quad t=\frac{\rho_{0}}{\eta\left(T_{0}\right)} t^{\prime},
$$

where $\boldsymbol{x}^{\prime}$ are spatial coordinates and $t^{\prime}$ is time.

The dot denotes the standard scalar product, while the overline indicates the symmetric traceless part of a tensor. For a tensor $\boldsymbol{a}=\left(a_{i j}\right)$ this part is

$$
\overline{\boldsymbol{a}}=\frac{1}{2}\left(\boldsymbol{a}+\boldsymbol{a}^{T}\right)-\frac{1}{3} I \operatorname{tr}(\boldsymbol{a}),
$$

where $I$ is unit matrix. In particular,

$$
\overline{\nabla \boldsymbol{u}}=\frac{1}{2}\left(\nabla \boldsymbol{u}+(\nabla \boldsymbol{u})^{T}-\frac{2}{3} I \nabla \cdot \boldsymbol{u}\right),
$$

where $I=\left(\delta_{i j}\right)$ is the identity matrix.

We also study a simple model of a coupling of the hydrodynamic variables, $\boldsymbol{u}$ and $p(p(\boldsymbol{x}, t)=\rho(\boldsymbol{x}, t)+T(\boldsymbol{x}, t))$, to the nonhydrodynamic variable $\boldsymbol{\sigma}$, the 3D linearized Grad equations for 10 moments $p, \boldsymbol{u}$, and $\boldsymbol{\sigma}$ :

$$
\begin{aligned}
\partial_{t} p & =-\frac{5}{3} \nabla \cdot \boldsymbol{u}, \\
\partial_{t} \boldsymbol{u} & =-\nabla p-\nabla \cdot \boldsymbol{\sigma}, \\
\partial_{t} \boldsymbol{\sigma} & =-2 \overline{\nabla \boldsymbol{u}}-\frac{1}{\epsilon} \boldsymbol{\sigma} .
\end{aligned}
$$

Here, the coefficient $\frac{5}{3}$ is the adiabatic exponent of the 3D ideal gas.

The simplest model and the starting point in our analysis is the reduction of system (1.5) to the functions that depend on one space coordinate $x$ with the velocity $\boldsymbol{u}$ oriented along the $x$ axis:

$$
\begin{aligned}
& \partial_{t} p=-\frac{5}{3} \partial_{x} u \\
& \partial_{t} u=-\partial_{x} p-\partial_{x} \sigma, \\
& \partial_{t} \sigma=-\frac{4}{3} \partial_{x} u-\frac{1}{\epsilon} \sigma,
\end{aligned}
$$

where $\sigma$ is the dimensionless $x x$-component of the stress tensor and the equation describes the unidirectional solutions of the previous system (1.5).

These equations are elements of the staircase of simplifications, from the Boltzmann equation to moment equations of various complexity, which was introduced by Grad 68] and elaborated further by many authors. In particular, Levermore proved hyperbolicity of the properly constructed moment equations [106]. This staircase forms the basis of Extended Irreversible Thermodynamics (EIT) [84.

1.3. Singular perturbation and separation of times in kinetics. The kinetic equations are singularly perturbed with a small parameter $\epsilon$ (the "Knudsen number"), and we are interested in the asymptotic properties of solutions when $\epsilon$ is small. The physical interpretation of the Knudsen number is the ratio of the "microscopic lengths" (for example, the mean free path) to the "macroscopic scale", where the solution changes significantly. Therefore, its definition depends on the properties of solutions. If the space derivatives are uniformly bounded, then we can study the asymptotic behavior $\epsilon \rightarrow 0$. But for some singular solutions this problem statement may be senseless. The simple illustration of rescaling with the erasing 
of $\epsilon$ gives the set of travelling automodel solutions for (1.1). If we look for them in a form $f=\varphi(\boldsymbol{\xi}, \boldsymbol{v})$ where $\boldsymbol{\xi}=(\boldsymbol{x}-\boldsymbol{c t}) / \epsilon$, then the equation for $\varphi(\boldsymbol{\xi}, \boldsymbol{v})$ does not depend on $\epsilon$ :

$$
(\boldsymbol{v}-\boldsymbol{c}) \cdot \nabla_{\xi} \phi=Q(\phi) .
$$

In general, $\epsilon$ may be considered as a variable that is neither small nor large, and the problem is to analyze the dependence of solutions on $\epsilon$.

For the Boltzmann equation (1.1) the collision term $Q(f)$ does not enter directly into the time derivatives of the hydrodynamic variables, $\rho=\int f \mathrm{~d} \boldsymbol{v}, \boldsymbol{u}=\int \boldsymbol{v} f \mathrm{~d} \boldsymbol{v}$ and $T=\int(\boldsymbol{v}-\boldsymbol{u})^{2} f \mathrm{~d} \boldsymbol{v}$ because, due to mass, momentum, and energy conservation laws,

$$
\int\left\{1 ; \boldsymbol{v} ;(\boldsymbol{v}-\boldsymbol{u})^{2}\right\} Q(f) \mathrm{d} \boldsymbol{v}=0 .
$$

The following dynamical system point of view is valid for smooth solutions in a bounded region with no-flux and equilibrium boundary conditions, but it is used with some success much more widely. The collision term is "fast" (includes the large parameter $1 / \varepsilon$ ) and does not affect the macroscopic hydrodynamic variables directly. Therefore, the following qualitative picture is expected for the solutions:

(i) The collision term goes quickly almost to its equilibrium (the system almost approaches a local equilibrium), and during this fast initial motion the changes of hydrodynamic variables are small.

(ii) After that the distribution function is defined with high accuracy by the hydrodynamic variables (if they have bounded space derivatives).

The relaxation of the collision term almost to its equilibrium is supported by monotonic entropy growth (Boltzmann's $H$-theorem). This qualitative picture is illustrated in Figure 1.

Such a "nonrigorous picture of the Boltzmann dynamics" 29 which operates by the manifolds in the space of probability distributions is a seminal tool for production of qualitative hypotheses. The points ("states") in Figure 1 correspond to the distributions $f(\boldsymbol{x}, \boldsymbol{v})$, and the points in the projection correspond to the hydrodynamic fields in space.

For Grad equations (1.2)-(1.3), (1.5), and (1.6), the hydrodynamic variables $\rho, \boldsymbol{u}, T$ are explicitly separated from the fluxes, and the projection onto the hydrodynamic fields is just the selection of the hydrodynamic part of the set of all fields. For example, for (1.6) this is just the selection of $p(\boldsymbol{x}), \boldsymbol{u}(\boldsymbol{x})$ from the whole set of fields $p(\boldsymbol{x}), \boldsymbol{u}(\boldsymbol{x}), \boldsymbol{\sigma}(\boldsymbol{x})$. The expected qualitative picture for smooth solutions is the same as in Figure 1 .

For finite-dimensional ODEs, Figure 1 represents the systems which satisfy the Tikhonov singular perturbation theorem [140]. In some formal sense, this picture for the Boltzmann equation is also rigorous when $\epsilon \rightarrow 0$, and it is proven in [6]. Assume that $f^{\epsilon}(t, \boldsymbol{x}, \boldsymbol{v})$ is a sequence of nonnegative solutions of the Boltzmann equation (1.1) when $\epsilon \rightarrow 0$ and there exists a limit $f^{\epsilon}(t, \boldsymbol{x}, \boldsymbol{v}) \rightarrow f^{0}(t, \boldsymbol{x}, \boldsymbol{v})$. Then (under some additional regularity conditions) this limit $f^{0}(t, \boldsymbol{x}, \boldsymbol{v})$ is local Maxwellian and the corresponding moments satisfy the compressible Euler equation. According to [126], this is "the easiest of all hydrodynamic limits of the Boltzmann equation at the formal level".

The theory of singular perturbations was developed starting from complex systems, from the Boltzmann equation (Hilbert [77, Enskog [35], Chapman 24], Grad [68, 69] ) to ODEs. The recently developed geometric theory of singular perturbation 


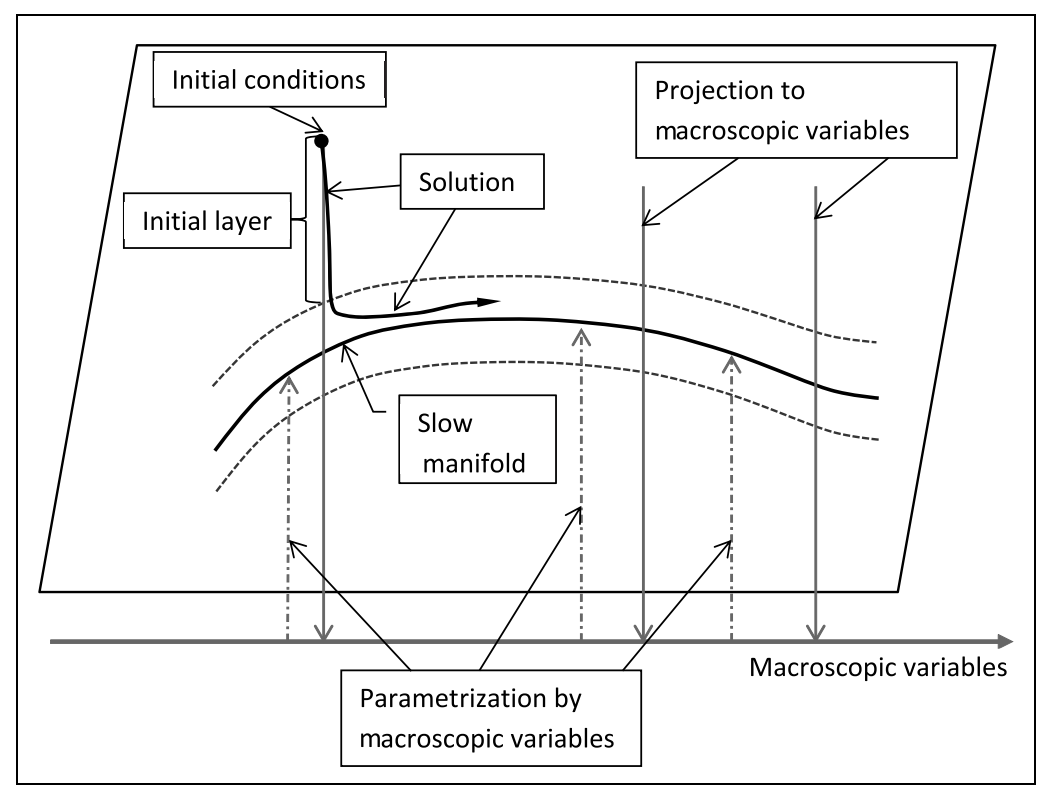

Figure 1. Fast-slow decomposition. Bold dashed lines outline the vicinity of the slow manifold where the solutions stay after the initial layer. The projection of the distributions onto the hydrodynamic fields and the parametrization of this manifold by the hydrodynamic fields are represented.

[36, 37, 83, can be considered as a formalization of the Chapman-Enskog approach for the area where complete rigorous theory is achievable.

A program of the derivation of (weak) solutions of the Navier-Stokes equations from the (weak) solutions of the Boltzmann equation was formulated in 1991 6] and finalized in 2004 [46] with the following answer: the incompressible NavierStokes (Navier-Stokes Fourier) equations appear in a limit of appropriately scaled solutions of the Boltzmann equation.

We use the geometry of time-separation (Figure 1) as a guide for formal constructions, and we present further development of this scheme using some ideas from thermodynamics and dynamics.

1.4. The structure of this paper. In Section 2 we introduce the invariance equation for invariant manifolds. It has been studied by Lyapunov (Lyapunov's auxiliary theorem [111, which we reproduce as Theorem 2.1 below). We describe the structure of the invariance equations for the Boltzmann and Grad equations, and in Section 2.2 we construct the Chapman-Enskog expansion for the solution of the invariance equation.

It may be worth stressing that the invariance equation is a nonlinear equation and there is no known general method to solve it even for linear differential equations. The main construction is illustrated on the simplest kinetic equation (1.6): in Section 2.3 the Euler, Navier-Stokes, Burnett, and super-Burnett terms are calculated for this equation, and the "ultraviolet catastrophe" of the Chapman-Enskog series is demonstrated (Figure 3). 
The first example of the exact summation of the Chapman-Enskog series is presented in detail for the simplest system (1.6) in Section 3. We analyze the structure of the Chapman-Enskog series and find the pseudodifferential representation of the stress tensor on the hydrodynamic invariant manifold. Using this representation, in Section 3.3 we represent the energy balance equation in the "capillarity-viscosity" form proposed by Slemrod [135. This form explains the macroscopic sense of the dissipation saturation effect: the attenuation rate does not depend on the wave vector $k$ for short waves (it tends to a constant value when $k^{2} \rightarrow \infty$ ). In the highly nonequilibrium gas, the capillarity energy becomes significant, and it tends to infinity for high-velocity gradients.

In the Fourier representation, the invariance equation for (1.6) is a system of two coupled quadratic equations with linear in $k^{2}$ coefficients (Section 3.4). It can be solved in radicals and the corresponding hydrodynamics has the acoustic waves decay with saturation (Section 3.5) . The hydrodynamic invariant manifold for (1.6) is analytic at the infinitely distant point $k^{2}=\infty$. Matching of the first terms of the Taylor series in powers of $1 / k^{2}$ with the first terms of the Chapman-Enskog series gives simpler hydrodynamic equations with qualitatively the same effects and even quantitatively the same saturation level of attenuation of acoustic waves (Section 3.6). We may guess that the matched asymptotics of this type include all the essential information about hydrodynamics, both at low and high frequencies.

The construction of the invariance equations in the Fourier representation remains the same for a general linear kinetic equation (Section 4.1). The exact hydrodynamics on invariant manifolds always inherits many important properties of the original kinetics, such as dissipation and conservation laws. In particular, if the original kinetic system is hyperbolic, then for bounded hydrodynamic invariant manifolds the hydrodynamic equations are also hyperbolic (Section 4.2).

In Section 4, we study invariance equations for three systems: one-dimensional solutions of the 13-moment Grad system (Section 4.3), the full three-dimensional 13-moment Grad system (Section 4.4), and the linearized BGK kinetic equation (Section 4.5). The 13-moment Grad system demonstrates an important effect: the invariance equation may lose the physically meaningful solution for short waves. Therefore, existence of the exact hydrodynamic manifold is not compulsory for all the usual kinetic equations. Nevertheless, for the BGK equation with the complete advection operator $\boldsymbol{v} \cdot \nabla$, the invariance equation exists for short waves also (as is demonstrated numerically in [87]).

For nonlinear kinetics, the exact solutions to invariance equations are not known. In Section 5 we demonstrate two approaches to approximate invariant manifolds. First, for the nonlinear Grad equation, we find the leading terms of the ChapmanEnskog series in the order of the Mach number and exactly sum them. For this purpose, we construct the approximate invariant manifold and find the solution for the nonlinear viscosity in the form of an ODE (Section 5.1). For one-dimensional solutions of the Boltzmann equation, we construct the invariance equation and demonstrate the result of the first Newton-Kantorovich iteration for the solution of this equation (Section 5.2 and [53,60]). Use of the approximate invariant manifolds causes a problem of dissipativity preservation in the hydrodynamics on these manifolds. There exists a unique modification of the projection operator that guarantees the preservation of entropy production for hydrodynamics produced by projection of kinetics onto an approximate invariant manifold even for rough approximations 
[59]. This construction is presented in Section [6. In the Conclusion, we discuss solved and unsolved problems and formulate several hypotheses.

\section{INVARIANCE EQUATION AND THE CHAPMAN-ENSKog EXPANSION}

2.1. The idea of an invariant manifold in kinetics. Very often, the ChapmanEnskog expansion for the Boltzmann equation is introduced as an asymptotic expansion in powers of $\epsilon$ of the solutions of equation (1.1), which should depend on time only through time dependence of the macroscopic hydrodynamic fields. Historically, the definition of the method is "procedure oriented": an expansion is created step by step with the leading idea that solutions should depend on time only through the macroscopic variables and their derivatives. In this approach what we are looking for often remains hidden.

The result of the Chapman-Enskog method is not a solution of the kinetic equation but rather the proper parametrization of microscopic variables (distribution functions) by the macroscopic (hydrodynamic) fields. It is a lifting procedure: we take the hydrodynamic fields and find for them the corresponding distribution function. This lifting should be consistent with the kinetics, i.e., the set of the corresponding distributions (collected for all possible hydrodynamic fields) should be invariant with respect to a shift in time. Therefore, the Chapman-Enskog procedure looks for an invariant manifold for the kinetic equation which is close to the local equilibrium for a small Knudsen number and smooth hydrodynamic fields with bounded derivatives. This is the "object oriented" description of the ChapmanEnskog procedure.

The puzzle in the statement of the problem of transition from kinetics to hydrodynamics has been so deep that Uhlenbeck called it the "Hilbert paradox" [142. In the reduced hydrodynamic description, the state of a gas is completely determined if one knows initially the space dependence of the five macroscopic variables $p, \boldsymbol{u}$, and $T$. Uhlenbeck has found this impossible:

"On the one hand, it couldn't be true because the initial-value problem for the Boltzmann equation (which supposedly gives a better description of the state of the gas) requires the knowledge of the initial value of the distribution function $f(\boldsymbol{r}, \boldsymbol{v}, t)$ of which $p, \boldsymbol{u}$, and $T$ are only the first five moments in $\boldsymbol{v}$. But on the other hand the hydrodynamical equations surely give a causal description of the motion of a fluid. Otherwise, how could fluid mechanics be used?"

Perhaps McKean gave the first clear explanation of the problem as a construction of a "nice submanifold" where "the hydrodynamical equations define the same flow as the (more complicated) Boltzmann equation does" [114. He presented the problem using a partially commutative diagram; we use this idea in slightly revised form in Figure 2,

The invariance equation just expresses the fact that the vector field is tangent to the manifold. The invariance equation has the simplest form for manifolds parametrized by moments, i.e., by the values of the given linear functionals. Let us consider an equation in a domain $U$ of a normed space $E$ with analytical (at least, Gateaux-analytical) right-hand sides

$$
\partial_{t} f=J(f) .
$$




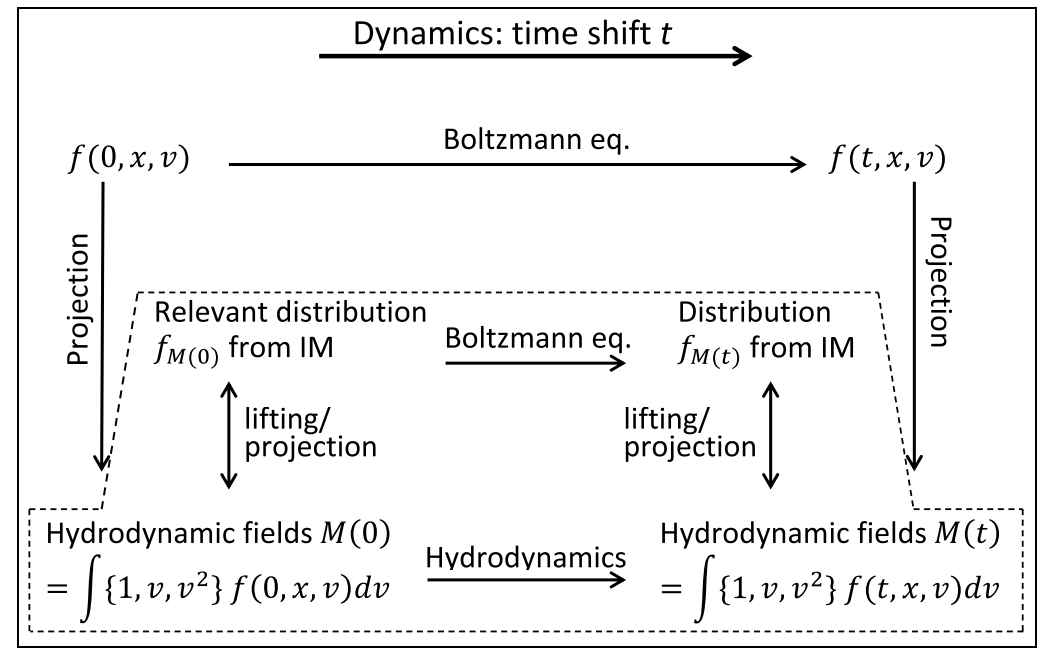

FiguRE 2. McKean diagram. The Chapman-Enskog procedure aims to create a lifting operation, from the hydrodynamic variables to the corresponding distributions on the invariant manifold. IM stands for invariant manifold. The part of the diagram in the dashed polygon is commutative.

A space of macroscopic variables (moment fields) is defined with a surjective linear map to them, $m: f \mapsto M$ ( $M$ are macroscopic variables). Below when referring to a manifold parametrized with the macroscopic fields $M$, we use the notation $\boldsymbol{f}_{M}$. We are looking for an invariant manifold $\boldsymbol{f}_{M}$ parametrized by the value of $M$, with the self-consistency condition $m\left(\boldsymbol{f}_{M}\right)=M$.

The invariance equation is

$$
J\left(\boldsymbol{f}_{M}\right)=\left(D_{M} \boldsymbol{f}_{M}\right) m\left(J\left(\boldsymbol{f}_{M}\right)\right) .
$$

Here, the differential $D_{M}$ of $\boldsymbol{f}_{M}$ is calculated at the point $M=m\left(\boldsymbol{f}_{M}\right)$.

Equation (2.2) means that the time derivative of $\boldsymbol{f}$ on the manifold $\boldsymbol{f}_{M}$ can be calculated by a simple chain rule: calculate the derivative of $M$ using the map $m$, $\dot{M}=m\left(J\left(\boldsymbol{f}_{M}\right)\right)$, and then write that the time dependence of $\boldsymbol{f}$ can be expressed through the time dependence of $M$. If we find the approximate solution to equation (2.2), then the approximate reduced model (hydrodynamics) is

$$
\partial_{t} M=m\left(J\left(\boldsymbol{f}_{M}\right)\right) .
$$

The invariance equation can be represented in the form

$$
\partial_{t}^{\text {micro }} \boldsymbol{f}_{M}=\partial_{t}^{\text {macro }} \boldsymbol{f}_{M},
$$

where the microscopic time derivative, $\partial_{t}^{\text {micro }} \boldsymbol{f}_{M}$, is just a value of the vector field $J\left(\boldsymbol{f}_{M}\right)$ and the macroscopic time derivative is calculated by the chain rule

$$
\partial_{t}^{\text {macro }} \boldsymbol{f}_{M}=\left(D_{M} \boldsymbol{f}_{M}\right) \partial_{t} M
$$

under the assumption that dynamics of $M$ follows the projected equation (2.3).

We use the natural (and naive) moment-based projection (2.3) until Section 6 where we demonstrate that in many situations the modified projectors are more suitable from the thermodynamic point of view. In addition, the flexible choice of 
projectors allows us to treat various nonlinear functionals (like scattering rates) as macroscopic variables [56, 61.

If $\boldsymbol{f}_{M}$ is a solution to the invariance equation (2.2), then the reduced model (2.3) has two important properties:

- Preservation of conservation laws. If a differentiable functional $U(f)$ is conserved due to the initial kinetic equation (2.1), then the functional $U_{M}=U\left(\boldsymbol{f}_{M}\right)$ conserves due to reduced system (2.3), i.e., it has zero time derivative due to this system.

- Preservation of dissipation. If a time derivative of a differentiable functional $H(f)$ is nonpositive due to the initial kinetic equation, then the time derivative of the functional $H_{M}=H\left(\boldsymbol{f}_{M}\right)$ is also nonpositive due to reduced system.

These elementary properties are the obvious consequences of the invariance equation (2.2) and the chain rule for differentiation. Indeed, for every differentiable functional $S(f)$ we introduce a functional $S_{M}=S\left(f_{M}\right)$. Then the time derivative of $S_{M}$ due to projected equation (2.3) coincides with the time derivative of $S(f)$ at point $f=\boldsymbol{f}_{M}$ due to (2.1). (Preservation of time derivatives.) Despite the very elementary character of these properties, they may be extremely important in construction of the energy and entropy formulas for the projected equations (2.3) and in the proof of the $H$-theorem and hyperbolicity.

The difficulties with preservation of conservation laws and dissipation inequalities may occur when one uses the approximate solutions of the invariance equation. For these situations, two techniques are invented: modification of the projection operation (see [51,59] and Section [6 below) and modification of the entropy functional [70,71]. They allow to retain the dissipation inequality for the approximate equations.

It is obvious that the invariance equation (2.2) for dynamical systems usually has too many solutions, at least locally, in a vicinity of any nonsingular point. For example, every trajectory of (2.1) is a one-dimensional invariant manifold, and if a manifold $\mathcal{L}$ is transversal to a vector field $J$, then the trajectory of $\mathcal{L}$ is invariant.

Lyapunov used the analyticity of the invariant manifold for the finite-dimensional analytic vector fields $J$ to prove its existence and uniqueness near a fixed point $\boldsymbol{f}_{0}$ if ker $m$ is a invariant subspace of the Jacobian $(D J)_{0}$ of $J$ at this point and under some "no resonance" conditions (the Lyapunov auxiliary theorem [111). Under these conditions, there exist many smooth nonanalytical manifolds, but the analytical one is unique.

Theorem 2.1 (Lyapunov auxiliary theorem). Let ker $m$ have a $(D J)_{0}$-invariant supplement $(\operatorname{ker} m)^{\prime}, E=\operatorname{ker} m \oplus(\operatorname{ker} m)^{\prime}$. Assume that the restriction $(D J)_{0}$ onto ker $m$ has the spectrum $\kappa_{1}, \ldots, \kappa_{j}$ and the restriction of this operator on the supplement $(\operatorname{ker} m)^{\prime}$ has the spectrum $\lambda_{1}, \ldots, \lambda_{l}$. Let the following two conditions hold:

(1) $0 \notin \operatorname{conv}\left\{\kappa_{1}, \ldots, \kappa_{j}\right\}$;

(2) the spectra $\left\{\kappa_{1}, \ldots, \kappa_{j}\right\}$ and $\left\{\lambda_{1}, \ldots, \lambda_{l}\right\}$ are not related by any equation of the form

$$
\sum_{i} n_{i} \kappa_{i}=\lambda_{k}
$$

with integer $n_{i}$. 
Then there exists a unique analytic solution $\boldsymbol{f}_{M}$ of the invariance equation (2.2) with condition $\boldsymbol{f}_{M}=\boldsymbol{f}_{0}$ for $M=m\left(\boldsymbol{f}_{0}\right)$, and in a sufficiently small vicinity of $m\left(\boldsymbol{f}_{0}\right)$.

This solution is tangent to $(\operatorname{ker} m)^{\prime}$ at point $\boldsymbol{f}_{0}$.

Recently, the approach to invariant manifolds based on the invariance equation in combination with the Lyapunov auxiliary theorem was used for the reduction of kinetic systems 9294 .

2.2. The Chapman-Enskog expansion. The Chapman-Enskog and geometric singular perturbation approach assume the special singularly perturbed structure of the equations and look for the invariant manifold in a form of the series in the powers of a small parameter $\epsilon$. A one-parametric system of equations is considered:

$$
\partial_{t} f+A(f)=\frac{1}{\epsilon} Q(f) .
$$

The following assumptions connect the macroscopic variables to the singular perturbation:

- $m(Q(f))=0$;

- for each $M \in m(U)$, the system of equations

$$
Q(f)=0, m(f)=M,
$$

has a unique solution $\boldsymbol{f}_{M}^{\mathrm{eq}}$ (in Boltzmann kinetics it is the local Maxwellian);

- $\boldsymbol{f}_{M}^{\mathrm{eq}}$ is asymptotically stable and globally attracting for the fast system

$$
\partial_{t} f=\frac{1}{\epsilon} Q(f)
$$

in $\left(\boldsymbol{f}_{M}^{\mathrm{eq}}+\operatorname{ker} m\right) \cap U$.

Let the differential of the fast vector field $Q(f)$ at equilibrium $\boldsymbol{f}_{M}^{\mathrm{eq}}$ be $\mathcal{Q}_{M}$. For the Chapman-Enskog method it is important that $\mathcal{Q}_{M}$ is invertible in ker $m$. For classical kinetic equations this assumption can be checked using the symmetry of $\mathcal{Q}_{M}$ with respect to the entropic inner product (Onsager's reciprocal relations).

The invariance equation for the singularly perturbed system (2.4) with the moment parametrization $m$ is:

$$
\frac{1}{\epsilon} Q\left(\boldsymbol{f}_{M}\right)=A\left(\boldsymbol{f}_{M}\right)-\left(D_{M} \boldsymbol{f}_{M}\right)\left(m\left(A\left(\boldsymbol{f}_{M}\right)\right)\right)
$$

The fast vector field vanishes on the right-hand side of this equation because $m(Q(\boldsymbol{f}))=0$. The self-consistency condition $m\left(\boldsymbol{f}_{M}\right)=M$ gives

$$
m\left(D_{M} \boldsymbol{f}_{M}\right) m(J)=m(J)
$$

for all $J$, hence,

$$
m\left[A\left(\boldsymbol{f}_{M}\right)-\left(D_{M} \boldsymbol{f}_{M}\right) m\left(A\left(\boldsymbol{f}_{M}\right)\right)\right]=0 .
$$

If we find an approximate solution of (2.5), then the corresponding macroscopic (hydrodynamic) equation (2.3) is

$$
\partial_{t} M+m\left(A\left(\boldsymbol{f}_{M}\right)\right)=0 .
$$

Let us represent all the operators in (2.5) by the Taylor series (recall that in the Boltzmann equation $A$ is the linear free-flight operator, $A=v \cdot \nabla$, and $Q$ is the 
quadratic collision operator). We look for the invariant manifold in the form of the power series

$$
\boldsymbol{f}_{M}=\boldsymbol{f}_{M}^{\mathrm{eq}}+\sum_{i=1}^{\infty} \epsilon^{i} \boldsymbol{f}_{M}^{(i)}
$$

with the self-consistency condition $m\left(\boldsymbol{f}_{M}\right)=M$, which implies $m\left(\boldsymbol{f}_{M}^{\mathrm{eq}}\right)=M$, $m\left(\boldsymbol{f}_{M}^{(i)}\right)=0$ for $i \geq 1$. After matching the coefficients of the series in (2.5), we obtain for every $\boldsymbol{f}_{M}^{(i)}$ a linear equation

$$
\mathcal{Q}_{M} \boldsymbol{f}_{M}^{(i)}=P^{(i)}\left(\boldsymbol{f}_{M}^{\mathrm{eq}}, \boldsymbol{f}_{M}^{(1)}, \ldots, \boldsymbol{f}_{M}^{(i-1)}\right),
$$

where the polynomial operator $P^{(i)}$ at each order $i$ can be obtained by straightforward calculations from (2.5). Due to self-consistency, $m\left(P^{(i)}\right)=0$ for all $i$, and the equation (2.9) is solvable. The first term of the Chapman-Enskog expansion has a simple form:

$$
\boldsymbol{f}_{M}^{(1)}=\mathcal{Q}_{M}^{-1}\left(1-\left(D_{M} \boldsymbol{f}_{M}^{\mathrm{eq}}\right) m\right)\left(A\left(\boldsymbol{f}_{M}^{\mathrm{eq}}\right)\right)
$$

A detailed analysis of explicit versions of this formula for the Boltzmann equation and other kinetic equations is presented in many books and papers [24,78. Most of the physical applications of kinetic theory, from the transport processes in gases to modern numerical methods (lattice Boltzmann models [136]) give examples of the practical applications and deciphering of this formula. For Boltzmann kinetics, the zero-order approximation, $\boldsymbol{f}_{M}^{(0)} \approx \boldsymbol{f}_{M}^{\mathrm{eq}}$, produces in projection on the hydrodynamic fields (2.7) the compressible Euler equation. The first-order approximate invariant manifold, $\boldsymbol{f}_{M}^{(1)} \approx \boldsymbol{f}_{M}^{\mathrm{eq}}+\epsilon \boldsymbol{f}_{M}^{(1)}$, gives the compressible Navier-Stokes equation and provides the explicit dependence of the transport coefficients from the collision model. This bridge from the "atomistic view to the laws of motion of continua" is, in some sense, the main result of the Boltzmann kinetics, and it follows precisely Hilbert's request, but not as rigorously as desired.

The calculation of higher-order terms needs nothing but differentiation and calculation of the inverse operator $\mathcal{Q}_{M}^{-1}$. (Nevertheless, these calculations may be very bulky, and one of the creators of the method, S. Chapman, compared reading his book 24] to "chewing glass", cited by [15.) Differentiability is needed also because the transport operator $A$ should be bounded to provide strong sense to the manipulations (see the discussion in [127]). The second order in $\epsilon$ hydrodynamic equations (2.3) are called Burnett equations (with $\epsilon^{2}$ terms) and super-Burnett equations for higher orders.

2.3. Euler, Navier-Stokes, Burnett, and super-Burnett terms for a simple kinetic equation. Let us illustrate the basic construction on the simplest example (1.6):

$$
\begin{gathered}
\boldsymbol{f}=\left(\begin{array}{l}
p(x) \\
u(x) \\
\sigma(x)
\end{array}\right), m=\left(\begin{array}{lll}
1 & 0 & 0 \\
0 & 1 & 0
\end{array}\right), M=\left(\begin{array}{l}
p(x) \\
u(x)
\end{array}\right), \operatorname{ker} m=\left\{\left(\begin{array}{l}
0 \\
0 \\
y
\end{array}\right)\right\}, \\
A(\boldsymbol{f})=\left(\begin{array}{c}
\frac{5}{3} \partial_{x} u \\
\partial_{x} p+\partial_{x} \sigma \\
\frac{4}{3} \partial_{x} u
\end{array}\right), Q(\boldsymbol{f})=\left(\begin{array}{c}
0 \\
0 \\
-\sigma
\end{array}\right), \mathcal{Q}_{M}^{-1}=\mathcal{Q}_{M}=-1 \text { on ker } m,
\end{gathered}
$$




$$
\boldsymbol{f}_{M}^{\mathrm{eq}}=\left(\begin{array}{c}
p(x) \\
u(x) \\
0
\end{array}\right), D_{M} \boldsymbol{f}_{M}^{\mathrm{eq}}=\left(\begin{array}{ll}
1 & 0 \\
0 & 1 \\
0 & 0
\end{array}\right), \boldsymbol{f}_{M}^{(1)}=\left(\begin{array}{c}
0 \\
0 \\
-\frac{4}{3} \partial_{x} u
\end{array}\right)
$$

We hasten to remark that (1.6) is a simple linear system and can be integrated immediately in explicit form. However, that solution contains both the fast and slow components, and it does not readily reveal the slow hydrodynamic manifold of the system. Instead, we are interested in extracting this slow manifold by a direct method. The Chapman-Enskog expansion is thus the tool for this extraction, which we shall address first.

The projected equations in the zeroth (Euler) and the first (Navier-Stokes) order of $\epsilon$ are

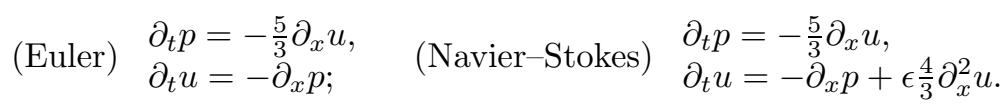

It is straightforward to calculate the two next terms (Burnett and super-Burnett ones), but let us introduce some convenient notation to represent the whole Chapman-Enskog series for (1.6). Only the third component of the invariance equation (2.5) for (1.6) is nontrivial because of the self-consistency condition (2.6), and we can write

$$
-\frac{1}{\epsilon} \sigma_{(p, u)}=\frac{4}{3} \partial_{x} u-\frac{5}{3}\left(D_{p} \sigma_{(p, u)}\right)\left(\partial_{x} u\right)-\left(D_{u} \sigma_{(p, u)}\right)\left(\partial_{x} p+\partial_{x} \sigma_{(p, u)}\right) .
$$

Here, $M=(p, u)$ and the differentials are calculated by the elementary rule: if a function $\Phi$ depends on values of $p(x)$ and its derivatives, $\Phi=\Phi\left(p, \partial_{x} p, \partial_{x}^{2} p, \ldots\right)$, then $D_{p} \Phi$ is a differential operator,

$$
D_{p} \Phi=\frac{\partial \Phi}{\partial p}+\frac{\partial \Phi}{\partial\left(\partial_{x} p\right)} \partial_{x}+\frac{\partial \Phi}{\partial\left(\partial_{x}^{2} p\right)} \partial_{x}^{2}+\cdots .
$$

The equilibrium of the fast system (the Euler approximation) is known, $\sigma_{(p, u)}^{(0)}=$ 0 . We have already found $\sigma_{(p, u)}^{(1)}=-\frac{4}{3} \partial_{x} u$ (the Navier-Stokes approximation). In each order of the Chapman-Enskog expansion $i \geq 1$, we get from (2.11)

$$
\sigma_{(p, u)}^{(i+1)}=\frac{5}{3}\left(D_{p} \sigma_{(p, u)}^{(i)}\right)\left(\partial_{x} u\right)+\left(D_{u} \sigma_{(p, u)}^{(i)}\right)\left(\partial_{x} p\right)+\sum_{j+l=i}\left(D_{u} \sigma_{(p, u)}^{(j)}\right)\left(\partial_{x} \sigma_{(p, u)}^{(l)}\right) .
$$

This chain of equations is nonlinear but every $\sigma_{(p, u)}^{(i+1)}$ is a linear function of derivatives of $u$ and $p$ with constant coefficients because this sequence starts from $-\frac{4}{3} \partial_{x} u$ and the induction step in $i$ is obvious. Let $\sigma_{(p, u)}^{(i)}$ be a linear function of derivatives of $u$ and $p$ with constant coefficients. Then its differentials $D_{p} \sigma_{(p, u)}^{(i)}$ and $D_{u} \sigma_{(p, u)}^{(i)}$ are linear differential operators with constant coefficients, and all terms in (2.12) are again linear functions of derivatives of $u$ and $p$ with constant coefficients.

For $\sigma_{(p, u)}^{(2)}(i+1=2)$ the operators in the right-hand part of (2.12) are $\left(D_{p} \sigma_{(p, u)}^{(1)}\right)=$ $0,\left(D_{u} \sigma_{(p, u)}^{(1)}\right)=-\frac{4}{3} \partial_{x}$, and in the third term in each summand either $l=0, j=1$ or $l=1, j=0$. Therefore, for the Burnett term,

$$
\sigma_{(p, u)}^{(2)}=-\frac{4}{3} \partial_{x}^{2} p .
$$


For the super-Burnett term in $\sigma_{(p, u)}^{(3)}(i+1=3)$, the operators in the right-hand part of (2.12) are $\left(D_{p} \sigma_{(p, u)}^{(2)}\right)=-\frac{4}{3} \partial_{x}^{2},\left(D_{u} \sigma_{(p, u)}^{(2)}\right)=0$, and in the third term the only summand with $l=j=1$ may take a nonzero value:

$$
\left(D_{u} \sigma_{(p, u)}^{(1)}\right)\left(\partial_{x} \sigma_{(p, u)}^{(1)}\right)=\left(-\frac{4}{3} \partial_{x}^{2}\right)\left(-\frac{4}{3} \partial_{x} u\right)=\frac{16}{9} \partial_{x}^{3} u .
$$

Finally, $\sigma_{(p, u)}^{(3)}=-\frac{4}{9} \partial_{x}^{3} u$ and the projected equations have the form

$$
\begin{aligned}
& \partial_{t} p=-\frac{5}{3} \partial_{x} u
\end{aligned}
$$

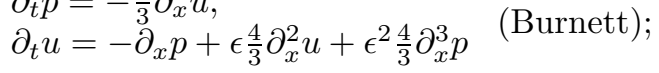

$$
\begin{aligned}
& \partial_{t} p=-\frac{5}{3} \partial_{x} u \\
& \partial_{t} u=-\partial_{x} p+\epsilon \frac{4}{3} \partial_{x}^{2} u+\epsilon^{2} \frac{4}{3} \partial_{x}^{3} p+\epsilon^{3} \frac{4}{9} \partial_{x}^{4} u \quad \text { (super Burnett). }
\end{aligned}
$$

To see the properties of the resulting equations, we compute the dispersion relation for the hydrodynamic modes. Using a new space-time scale, $x^{\prime}=\epsilon^{-1} x$, and $t^{\prime}=\epsilon^{-1} t$, and representing $u=u_{k} \varphi\left(x^{\prime}, t^{\prime}\right)$, and $p=p_{k} \varphi\left(x^{\prime}, t^{\prime}\right)$, where $\varphi\left(x^{\prime}, t^{\prime}\right)=$ $\exp \left(\omega t^{\prime}+i k x^{\prime}\right)$, and $k$ is a real-valued wave vector, we obtain the following dispersion relations $\omega(k)$ from the condition of a nontrivial solvability of the corresponding linear system with respect to $u_{k}$ and $p_{k}$ :

$$
\omega_{ \pm}=-\frac{2}{3} k^{2} \pm \frac{1}{3} i|k| \sqrt{15-4 k^{2}}
$$

for the Navier-Stokes approximation;

$$
\omega_{ \pm}=-\frac{2}{3} k^{2} \pm \frac{1}{3} i|k| \sqrt{15+16 k^{2}}
$$

for the Burnett approximation (2.13); and

$$
\omega_{ \pm}=\frac{2}{9} k^{2}\left(k^{2}-3\right) \pm \frac{1}{9} i|k| \sqrt{135+144 k^{2}+24 k^{4}-4 k^{6}}
$$

for the super-Burnett approximation (2.14).

These examples demonstrate that the real part is nonpositive, $\operatorname{Re}\left(\omega_{ \pm}(k)\right) \leq 0$ (Figure 3), for the Navier-Stokes (2.15) and for the Burnett (2.16) approximations, for all wave vectors. Thus, these approximations describe attenuating acoustic waves. However, for the super-Burnett approximation, the function $\operatorname{Re}\left(\omega_{ \pm}(k)\right)$ (2.17) becomes positive as soon as $|k|>\sqrt{3}$. The equilibrium is stable within the Navier-Stokes and the Burnett approximation, and it becomes unstable within the super-Burnett approximation for sufficiently short waves. Similar to the case of the Bobylëv instability of the Burnett hydrodynamics for the Boltzmann equation, the latter result contradicts the dissipative properties of the Grad system (1.6): the spectrum of the kinetic system (1.6) is stable for arbitrary $k$ (see Figure 3). For the 13-moment system (1.2)-(1.3) the instability of short waves appears already in the Burnett approximation [60, 90] (see Section 3 below). For the Boltzmann equation this effect was discovered by Bobylëv [9]. In Figure 3, we also represent the attenuation rates of the hydrodynamic and nonhydrodynamic mode of the kinetic equations (1.6). The characteristic equation of these kinetic equations reads

$$
3 \omega^{3}+3 \omega^{2}+9 k^{2} \omega+5 k^{2}=0 .
$$

The two complex-conjugate roots of this equation correspond to the hydrodynamic modes, while for the nonhydrodynamic real mode, $\omega_{n h}(k), \omega_{n h}(0)=-1$, and $\omega_{n h} \rightarrow-0.5$ as $|k| \rightarrow \infty$. The nonhydrodynamic modes of the Grad equations 


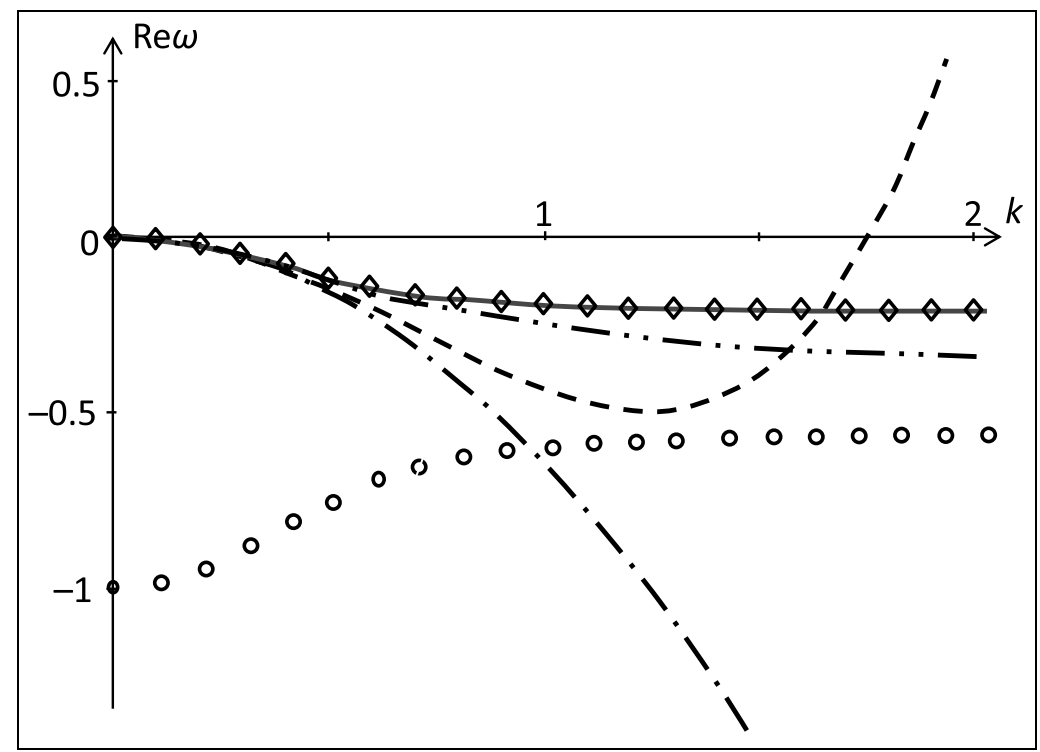

Figure 3. Attenuation rates [90]. Solid: exact summation; diamonds: hydrodynamic modes of the kinetic equations with $\epsilon=1$ (1.6) (they match the solid line per construction); circles: the nonhydrodynamic mode of (1.6), $\epsilon=1$; dash-dot line: the NavierStokes approximation; dash: the super-Burnett approximation; dash-double-dot line: the first Newton iteration (3.19). The result for the second iteration (3.20) is indistinguishable from the exact solution at this scale.

are characterized by the common property that for them $\omega(0) \neq 0$. These modes are irrelevant to the Chapman-Enskog branch of the invariant manifold.

Thus, the Chapman-Enskog expansion

- gives excellent, but already known on phenomenological grounds, zero-, and first-order approximations - the Euler and Navier-Stokes equations;

- provides a bridge from microscopic models of collisions to macroscopic transport coefficients in the known continuum equations;

- already the next correction, not known phenomenologically and hence of interest, does not exist because of nonphysical behavior.

The first term of the Chapman-Enskog expansion gives the possibility of evaluating the coefficients in the phenomenological equations (such as viscosity, thermal conductivity, and diffusion coefficient) from the microscopic models of collisions. The success of the first-order approximation (2.10) is compatible with the failure of the higher-order terms. The Burnett and super-Burnett equations have nonphysical properties, negative viscosity for high gradients, and instability for short waves. The Chapman-Enskog expansion has to be truncated after the first-order term or not truncated at all.

Such a situation, when the first approximations are useful but the higher terms become senseless, is not very novel. There are at least three famous examples: 
- The "ultraviolet catastrophe" in higher-order terms because of physical phenomena at very short distances 33 and the perturbation series divergencies [146] are well known in quantum field theory, and many approaches have been developed to deal with these singularities [145.

- Singularities and divergence in the semi-classical Wentzel-Kramers-Brillouin (WKB) approach [42,81,144.

- The small denominators affect the convergence of the Poincaré series in the classical many-body problem and the theory of nearly integrable systems. They may even make the perturbation series approach senseless [3].

Many ideas have been proposed and implemented to deal with these singularities: use of the direct iteration method instead of power series in KAM [2, 3, 97]; renormalization [21,39, 116; summation and partial summation and rational approximation of the perturbation series [34, 113]; and string theories [28, 143] in quantum field theory [145]. Various ad hoc analytical and numerical regularization tricks have been proposed also. Exactly solvable models give the possibility of exhaustive analysis of the solutions. Even in the situation when they are not applicable directly to reality, we can use them as benchmarks for all perturbation and approximation methods and for regularization tricks.

We follow this stream of ideas with the modifications required for kinetic theory. In the next section we describe algebraic invariant manifolds for the kinetic equations (1.2)-(1.3), (1.5), (1.6), and demonstrate the exact summation approach for the Chapman-Enskog series for these models. We use these models to demonstrate the application of the Newton method to the invariance equation (2.5).

\section{Algebraic hydrodynamic invariant manifolds AND EXACT SUMMATION OF THE CHAPMAN-ENSKOG SERIES FOR THE SIMPLEST KINETIC MODEL}

3.1. Grin of the vanishing cat: $\epsilon=1$. At the end of the previous section we introduced a new space-time scale, $x^{\prime}=\epsilon^{-1} x$, and $t^{\prime}=\epsilon^{-1} t$. The rescaled equations do not depend on $\epsilon$ at all and are, at the same time, equivalent to the original systems. Therefore, the presence of the small parameter in the equations is virtual. "Putting $\epsilon$ back $=1$, you hope that everything will converge and single out a nice submanifold" [114.

In this section, we find the invariant manifold for the equations with $\epsilon=1$. Now, there is no fast-slow decomposition of motion. It is natural to ask: What is the remainder of the qualitative picture of slow invariant manifold presented in Figure 1? Or an even sharper question: What we are looking for?

The rest of the fast-slow decomposition is the zeroth term in the ChapmanEnskog expansion (2.8). It starts from the equilibrium of the fast motion, $\boldsymbol{f}_{M}^{\mathrm{eq}}$. This (local) equilibrium manifold corresponds to the limit $\epsilon=0$. The first terms of the series for $\sigma$ for (1.6),

$$
\sigma=-\epsilon \frac{4}{3} \partial_{x} u-\epsilon^{2} \frac{4}{3} \partial_{x}^{2} p-\epsilon^{3} \frac{4}{9} \partial_{x}^{3} u+\cdots,
$$

also bear the imprint of the zeroth approximation, $\sigma^{(0)}=0$, even when we take $\epsilon=1$. The Chapman-Enskog procedure derives recurrently terms of the series from the starting term $\boldsymbol{f}_{M}^{\mathrm{eq}}$. 
The problem of the invariant manifold includes two difficulties: (i) it is difficult to find any global solution or even prove its existence; and (ii) there often exists too many different local solutions. The auxiliary Lyapunov theorem gives the first solution of the problem near an equilibrium and several seminal hints for further attempts. One of them: Use the analyticity as a selection criterion. The ChapmanEnskog method demonstrates that the inclusion of the system in the one-parametric family (parametrized by $\epsilon$ ) and the requirement of analyticity up to the limit $\epsilon=0$ allows us to select a sensible solution to the invariance equation. Even if we return to a single system with $\epsilon=1$, the structure of the constructed invariant manifold remembers the limit case $\epsilon=0 \cdots$. This can be considered as a manifestation of the effect of the grin of the vanishing cat: "I've often seen a cat without a grin," thought Alice: "but a grin without a cat! It's the most curious thing I ever saw in my life!" [Lewis Carroll, Alice's Adventures in Wonderland]. The small parameter disappears (we take $\epsilon=1$ ), but the effect of its presence persists in the analytic invariant manifold. There are some other effects of such a grin in kinetics [66].

The use of the term "slow manifold" for the case $\epsilon=1$ seems to be an abuse of language. Nevertheless, this manifold has some imprints of slowness, at least for smooth solutions bounded by small number. The definition of slow manifolds for a single system may be a nontrivial task [27,60]. There is a problem with a local definition because for a given vector field, the "slowness" of a submanifold cannot be invariant with respect to diffeomorphisms in a vicinity of a regular point. Therefore, we use the term "hydrodynamic invariant manifold".

3.2. The pseudodifferential form of the stress tensor. Let us return to the simplest kinetic equation (1.6). In order to construct the exact solution, we first analyze the global structure of the Chapman-Enskog series given by the recurrence formula (2.12). The first three terms (3.1) give us a hint: the terms in the series alternate. For odd $i=1,3, \ldots$, they are proportional to $\partial_{x}^{i} u$, and for even $i=$ $2,4, \ldots$, they are proportional to $\partial_{x}^{i} p$. Indeed, this structure can be proved by induction on $i$ starting in (2.12) from the first term $-\frac{4}{3} \partial_{x} u$. It is sufficient to notice that $\left(D_{p} \partial_{x}^{(i)} p\right)=\partial_{x}^{(i)},\left(D_{p} \partial_{x}^{i} u\right)=0,\left(D_{u} \partial_{x}^{i} p\right)=0,\left(D_{u} \partial_{x}^{i} u\right)=\partial_{x}^{(i)}$ and to use the induction assumption in (2.12).

The global structure of the Chapman-Enskog series gives the following representation of the stress $\sigma$ on the hydrodynamic invariant manifold

$$
\sigma(x)=A\left(-\partial_{x}^{2}\right) \partial_{x} u(x)+B\left(-\partial_{x}^{2}\right) \partial_{x}^{2} p(x),
$$

where $A(y), B(y)$ are yet unknown functions and the sign "-" in the arguments is adopted for simplicity of formulas in the Fourier transform.

It is easy to prove the structure (3.2) without any calculation or induction. Let us use the symmetry property of the kinetic equation (1.6): it is invariant with respect to the transformation $x \mapsto-x, u \mapsto-u, p \mapsto p$ and $\sigma \mapsto \sigma$ which transforms solutions into solutions. The invariance equation inherits this property, the initial equilibrium $(\sigma=0)$ is also symmetric and, therefore, the expression for $\sigma(x)$ should be even. This is exactly (3.2) where $A(y)$ and $B(y)$ are arbitrary even functions. (If they are, say, twice differentiable at the origin, then we can represent them as functions of $y^{2}$.)

3.3. The energy formula and "capillarity" of ideal gas. Traditionally, $\sigma$ is considered as a viscous stress tensor, but the second term, $B\left(-\partial_{x}^{2}\right) \partial_{x}^{2} p(x)$, is 
proportional to second derivative of $p(x)$, and it does not meet usual expectations $(\sigma \sim \nabla u)$. Slemrod [134, 135] noticed that the proper interpretation of this term is the capillarity tension rather than viscosity. This is made clear by inspection of the energy balance formula. Let us derive the Slemrod energy formula for the simple model (1.6). The time derivative of the kinetic energy due to the first two equations (1.6) is

$$
\begin{aligned}
\frac{1}{2} \partial_{t} \int_{-\infty}^{\infty} u^{2} \mathrm{~d} x & =\int_{-\infty}^{\infty} u \partial_{t} u \mathrm{~d} x=-\int_{-\infty}^{\infty} u \partial_{x} p \mathrm{~d} x-\int_{-\infty}^{\infty} u \partial_{x} \sigma \mathrm{d} x \\
& =-\frac{1}{2} \partial_{t} \frac{3}{5} \int_{-\infty}^{\infty} p^{2} \mathrm{~d} x+\int_{-\infty}^{\infty} \sigma \partial_{x} u \mathrm{~d} x
\end{aligned}
$$

Here we used integration by parts and assumed that all the fields with their derivatives tend to 0 when $x \rightarrow \pm \infty$.

In $x$-space the energy formula is

$$
\frac{1}{2} \partial_{t}\left(\frac{3}{5} \int_{-\infty}^{\infty} p^{2} \mathrm{~d} x+\int_{-\infty}^{\infty} u^{2} \mathrm{~d} x\right)=\int_{-\infty}^{\infty} \sigma \partial_{x} u \mathrm{~d} x
$$

This form of the energy equation is standard. Note that the usual factor $\rho$ in front of $u^{2}$ is absent because we work with the linearized equations.

Let us use in (3.4) the representation (3.2) for $\sigma$ and notice that $\partial_{x} u=-\frac{3}{5} \partial_{t} p$ :

$$
\int_{-\infty}^{\infty} \sigma \partial_{x} u \mathrm{~d} x=\int_{-\infty}^{\infty} A\left(-\partial_{x}^{2}\right) \partial_{x}^{2} u \mathrm{~d} x-\frac{3}{5} \int_{-\infty}^{\infty}\left(\partial_{t} p\right)\left[B\left(-\partial_{x}^{2}\right) \partial_{x}^{2} p\right] \mathrm{d} x
$$

The operator $B\left(-\partial_{x}^{2}\right) \partial_{x}^{2}$ is symmetric; therefore,

$$
\int_{-\infty}^{\infty}\left(\partial_{t} p\right)\left[B\left(-\partial_{x}^{2}\right) \partial_{x}^{2} p\right] \mathrm{d} x=\frac{1}{2} \partial_{t}\left(\int_{-\infty}^{\infty} p\left[B\left(-\partial_{x}^{2}\right) \partial_{x}^{2} p\right] \mathrm{d} x\right)
$$

The quadratic form,

$$
U_{c}=\frac{3}{5} \int_{-\infty}^{\infty} p\left(B\left(-\partial_{x}^{2}\right) \partial_{x}^{2} p\right) \mathrm{d} x=-\frac{3}{5} \int_{-\infty}^{\infty}\left(\partial_{x} p\right)\left(B\left(-\partial_{x}^{2}\right) \partial_{x} p\right) \mathrm{d} x
$$

may be considered as a part of the energy. Moreover, if the function $B(y)$ is negative, then this form is positive. Due to Parseval's identity we have

$$
U_{c}=-\frac{3}{5} \int_{-\infty}^{\infty} k^{2} B\left(k^{2}\right)\left|p_{k}\right|^{2} \mathrm{~d} k .
$$

Finally, the energy formula in $x$-space is

$$
\begin{aligned}
\frac{1}{2} \partial_{t} \int_{-\infty}^{\infty}\left(\frac{3}{5} p^{2}+u^{2}\right. & \left.-\frac{3}{5}\left(\partial_{x} p\right)\left(B\left(-\partial_{x}^{2}\right) \partial_{x} p\right)\right) \mathrm{d} x \\
& =\int_{-\infty}^{\infty}\left(\partial_{x} u\right)\left(A\left(-\partial_{x}^{2}\right) \partial_{x} u\right) \mathrm{d} x
\end{aligned}
$$

In $k$-space it has the form

$$
\frac{1}{2} \partial_{t} \int_{-\infty}^{\infty}\left(\frac{3}{5}\left|p_{k}\right|^{2}+\left|u_{k}\right|^{2}-\frac{3}{5} k^{2} B\left(k^{2}\right)\left|p_{k}\right|^{2}\right) \mathrm{d} k=\int_{-\infty}^{\infty} k^{2} A\left(k^{2}\right)\left|u_{k}\right|^{2} \mathrm{~d} k .
$$

It is worth mentioning that the functions $A\left(k^{2}\right)$ and $B\left(k^{2}\right)$ are negative (see Section 3.4). If we keep only the first nontrivial terms, $A=B=-\frac{4}{3}$, then the energy 
formula becomes

$$
\begin{aligned}
& \frac{1}{2} \partial_{t} \int_{-\infty}^{\infty}\left(\frac{3}{5} p^{2}+u^{2}+\frac{4}{5}\left(\partial_{x} p\right)^{2}\right) \mathrm{d} x=-\frac{4}{3} \int_{-\infty}^{\infty}\left(\partial_{x} u\right)^{2} \mathrm{~d} x \\
& \frac{1}{2} \partial_{t} \int_{-\infty}^{\infty}\left(\frac{3}{5}\left|p_{k}\right|^{2} \mathrm{~d} k+\left|u_{k}\right|^{2}+\frac{4}{5} k^{2}\left|p_{k}\right|^{2}\right) \mathrm{d} k=-\frac{4}{3} \int_{-\infty}^{\infty} k^{2}\left|u_{k}\right|^{2} \mathrm{~d} k .
\end{aligned}
$$

Slemrod represents the structure of the obtained energy formula as

$$
\begin{array}{r}
\partial_{t}(\text { MECHANICAL ENERGY })+\partial_{t}(\text { CAPILLARITY ENERGY }) \\
=\text { VISCOUS DISSIPATION } .
\end{array}
$$

The capillarity terms $\left(\partial_{x} p\right)^{2}$ in the energy density are standard in the thermodynamics of phase transitions.

The bulk capillarity terms in fluid mechanics were introduced into the NavierStokes equations by Korteweg [99] (for a review of some further results see [32]). Such terms appear naturally in theories of the phase transitions, such as van der Waals liquids [131, Ginzburg-Landau [1, and Cahn-Hilliard equations [16, 17, and phase fields models 25. Surprisingly, such terms are also found in the ideal gas dynamics as a consequence of the Chapman-Enskog expansion 132, 133. In higher-order approximations, the viscosity is reduced by the terms which are similar to Korteweg's capillarity. Finally, in the energy formula for the exact sum of the Chapman-Enskog expansion, we see terms of the same form: the viscous dissipation is decreased and the additional term appears in the energy (3.7), (3.8).

3.4. Algebraic invariant manifold in Fourier representation. It is convenient to work with the pseudodifferential operators like (3.2) in Fourier space. Let us denote $p_{k}, u_{k}$, and, $\sigma_{k}$, where $k$ is the "wave vector" (space frequency).

The Fourier-transformed kinetic equation (1.6) takes the form $(\epsilon=1)$ :

$$
\begin{aligned}
\partial_{t} p_{k} & =-\frac{5}{3} i k u_{k} \\
\partial_{t} u_{k} & =-i k p_{k}-i k \sigma_{k}, \\
\partial_{t} \sigma_{k} & =-\frac{4}{3} i k u_{k}-\sigma_{k} .
\end{aligned}
$$

We know already that the result of the reduction should be a function $\sigma_{k}\left(u_{k}, p_{k}, k\right)$ of the following form:

$$
\sigma_{k}\left(u_{k}, p_{k}, k\right)=i k A\left(k^{2}\right) u_{k}-k^{2} B\left(k^{2}\right) p_{k},
$$

where $A$ and $B$ are unknown real-valued functions of $k^{2}$.

The question of the summation of the Chapman-Enskog series amounts to finding the two functions $A\left(k^{2}\right)$ and $B\left(k^{2}\right)$. Let us write the invariance equation for unknown functions $A$ and $B$. We can compute the time derivative of $\sigma_{k}\left(u_{k}, p_{k}, k\right)$ in two different ways. First, we use the right-hand side of the third equation in (3.12). We find the microscopic time derivative,

$$
\partial_{t}^{\text {micro }} \sigma_{k}=-i k\left(\frac{4}{3}+A\right) u_{k}+k^{2} B p_{k}
$$


Secondly, let us use the chain rule and the first two equations in (3.12). We find the macroscopic time derivative:

$$
\begin{aligned}
\partial_{t}^{\text {macro }} \sigma_{k} & =\frac{\partial \sigma_{k}}{\partial u_{k}} \partial_{t} u_{k}+\frac{\partial \sigma_{k}}{\partial p_{k}} \partial_{t} p_{k} \\
& =i k A\left(-i k p_{k}-i k \sigma_{k}\right)-k^{2} B\left(-\frac{5}{3} i k u_{k}\right) \\
& =i k\left(\frac{5}{3} k^{2} B+k^{2} A\right) u_{k}+k^{2}\left(A-k^{2} B\right) p_{k} .
\end{aligned}
$$

The microscopic time derivative should coincide with the macroscopic time derivative for all values of $u_{k}$ and $p_{k}$. This is the invariance equation:

$$
\partial_{t}^{\text {macro }} \sigma_{k}=\partial_{t}^{\text {micro }} \sigma_{k} \text {. }
$$

For the kinetic system (3.12), it reduces to a system of two quadratic equations for functions $A\left(k^{2}\right)$ and $B\left(k^{2}\right)$ :

$$
\begin{aligned}
& F(A, B, k)=-A-\frac{4}{3}-k^{2}\left(\frac{5}{3} B+A^{2}\right)=0, \\
& G(A, B, k)=-B+A\left(1-k^{2} B\right)=0 .
\end{aligned}
$$

The Taylor series for $A\left(k^{2}\right), B\left(k^{2}\right)$ correspond exactly to the Chapman-Enskog series: if we look for these functions in the form $A(y)=\sum_{l \geq 0} a_{l} y^{l}$ and $B(y)=$ $\sum_{l>0} b_{l} y^{l}$, then from (3.17) we find immediately $a_{0}=b_{0}=-\frac{4}{3}$ (these are exactly the Navier-Stokes and Burnett terms) and the recurrence equation for $a_{i+1}, b_{i+1}$ :

$$
\begin{aligned}
& a_{n+1}=\frac{5}{3} b_{n}+\sum_{m=0}^{n} a_{n-m} a_{m}, \\
& b_{n+1}=a_{n+1}+\sum_{m=0}^{n} a_{n-m} b_{m} .
\end{aligned}
$$

The initial condition for this set of equations are the Navier-Stokes and the Burnett terms $a_{0}=b_{0}=-\frac{4}{3}$.

The Newton method for the invariance equation (3.17) generates the sequence $A_{i}\left(k^{2}\right), B_{i}\left(k^{2}\right)$, where the differences, $\delta A_{i+1}=A_{i+1}-A_{i}$ and $\delta B_{i+1}=B_{i+1}-B_{i}$ satisfy the system of linear equations

$$
\left(\begin{array}{cc}
\left.\frac{\partial F\left(A, B, k^{2}\right)}{\partial A}\right|_{\left(A_{i}, B_{i}\right)} & \left.\frac{\partial F\left(A, B, k^{2}\right)}{\partial B}\right|_{\left(A_{i}, B_{i}\right)} \\
\left.\frac{\partial G\left(A, B, k^{2}\right)}{\partial A}\right|_{\left(A_{i}, B_{i}\right)} & \left.\frac{\partial G\left(A, B, k^{2}\right)}{\partial B}\right|_{\left(A_{i}, B_{i}\right)}
\end{array}\right)\left(\begin{array}{c}
\delta A_{i+1} \\
\delta B_{i+1}
\end{array}\right)+\left(\begin{array}{c}
F\left(A_{i}, B_{i}, k^{2}\right) \\
G\left(A_{i}, B_{i}, k^{2}\right)
\end{array}\right)=0 .
$$

Rewrite this system in the explicit form:

$$
\left(\begin{array}{cc}
-\left(1+2 k^{2} A_{i}\right) & -\frac{5}{3} k^{2} \\
1-k^{2} B_{i} & -\left(1+k^{2} A_{i}\right)
\end{array}\right)\left(\begin{array}{l}
\delta A_{i+1} \\
\delta B_{i+1}
\end{array}\right)+\left(\begin{array}{c}
F\left(A_{i}, B_{i}, k^{2}\right) \\
G\left(A_{i}, B_{i}, k^{2}\right)
\end{array}\right)=0 .
$$

Let us start from the zeroth-order term of the Chapman-Enskog expansion (Euler's approximation), $A_{0}=B_{0}=0$. Then, the first Newton iteration gives

$$
A_{1}=B_{1}=-\frac{4}{3+5 k^{2}} \text {. }
$$


The second Newton iteration also gives the negative rational functions

$$
\begin{aligned}
& A_{2}=-\frac{4\left(27+63 k^{2}+153 k^{2} k^{2}+125 k^{2} k^{2} k^{2}\right)}{3\left(3+5 k^{2}\right)\left(9+9 k^{2}+67 k^{2} k^{2}+75 k^{2} k^{2} k^{2}\right)}, \\
& B_{2}=-\frac{4\left(9+33 k^{2}+115 k^{2} k^{2}+75 k^{2} k^{2} k^{2}\right)}{\left(3+5 k^{2}\right)\left(9+9 k^{2}+67 k^{2} k^{2}+75 k^{2} k^{2} k^{2}\right)} .
\end{aligned}
$$

The corresponding attenuation rates are shown in Figure 3 . They are stable and converge fast to the exact solutions. At the infinity, $k^{2} \rightarrow \infty$, the second iteration has the same limit, as the exact solution: $k^{2} A_{2} \rightarrow-\frac{4}{9}$ and $k^{2} B_{2} \rightarrow-\frac{4}{5}$ (compare to Section 3.6).

Thus, we made three steps:

(1) We used the invariance equation, the Chapman-Enskog procedure, and the symmetry properties to find a linear space where the hydrodynamic invariant manifold is located. This space is parametrized by two functions of one variable (3.13).

(2) We used the invariance equation and defined an algebraic manifold in this space. For the simple kinetic system (1.6), (3.12) this manifold is given by the system of two quadratic equations which depends linearly on $k^{2}$ (3.17).

(3) We found that the Newton iterations for the invariant manifold demonstrate much better approximation properties than the truncated ChapmanEnskog procedure.

3.5. Stability of the exact hydrodynamic system and saturation of dissipation for short waves. Stability is one of the first questions to analyze. There exists a series of simple general statements about the preservation of stability, wellposedness, and hyperbolicity in the exact hydrodynamics. Indeed, any solution of the exact hydrodynamics is a projection of a solution of the initial equation from the invariant manifold onto the hydrodynamic moments (Figures 1 and 2) and the projection of a bounded solution is bounded. (In infinite dimension we have to assume that the projection is continuous with respect to the chosen norms.) Several statements of this type are discussed in Section 4. Nevertheless, a direct analysis of dispersion relations and attenuation rates is instructive. Knowing $A\left(k^{2}\right)$ and $B\left(k^{2}\right)$, the dispersion relation for the hydrodynamic modes can be derived:

$$
\omega_{ \pm}=\frac{k^{2} A}{2} \pm i \frac{|k|}{2} \sqrt{\frac{20}{3}\left(1-k^{2} B\right)-k^{2} A^{2}} .
$$

It is convenient to reduce the consideration to a single function. Solving the system (3.17) for $B$, and introducing a new function, $X\left(k^{2}\right)=k^{2} B\left(k^{2}\right)$, we obtain an equivalent cubic equation:

$$
-\frac{5}{3}(X-1)^{2}\left(X+\frac{4}{5}\right)=\frac{X}{k^{2}} .
$$

Since the hydrodynamic manifold should be represented by the real-valued functions $A\left(k^{2}\right)$ and $B\left(k^{2}\right)$ (3.13), we are only interested in the real-valued roots of (3.22).

An elementary analysis gives that the real-valued root $X\left(k^{2}\right)$ of $(3.22)$ is unique and negative for all finite values $k^{2}$. Moreover, the function $X\left(k^{2}\right)$ is a monotonic function of $k^{2}$. The limiting values are

$$
\lim _{|k| \rightarrow 0} X\left(k^{2}\right)=0, \quad \lim _{|k| \rightarrow \infty} X\left(k^{2}\right)=-0.8 .
$$


Under the conditions just mentioned, the function under the root in (3.21) is negative for all values of the wave vector $k$, including the limits, and we come to the dispersion law,

$$
\omega_{ \pm}=\frac{X}{2(1-X)} \pm i \frac{|k|}{2} \sqrt{\frac{5 X^{2}-16 X+20}{3}},
$$

where $X=X\left(k^{2}\right)$ is the real-valued root of equation (3.22). Since $0>X\left(k^{2}\right)>-1$ for all $|k|>0$, the attenuation rate, $\operatorname{Re}\left(\omega_{ \pm}\right)$, is negative for all $|k|>0$, and the exact acoustic spectrum of the Chapman-Enskog procedure is stable for arbitrary wave lengths (Figure 3, solid line). In the short-wave limit, from (3.24) we obtain

$$
\lim _{|k| \rightarrow \infty} \operatorname{Re} \omega_{ \pm}=-\frac{2}{9}, \quad \lim _{|k| \rightarrow \infty} \frac{\operatorname{Im} \omega_{ \pm}}{|k|}= \pm \sqrt{3} .
$$

3.6. Expansion at $k^{2}=\infty$ and matched asymptotics. For large values of $k^{2}$, a version of the Chapman-Enskog expansion at an infinitely distant point is useful. Let us rewrite the algebraic equation for the invariant manifold (3.17) in the form

$$
\begin{aligned}
\frac{5}{3} B+A^{2} & =-\varsigma\left(\frac{4}{3}+A\right), \\
A B & =\varsigma(A-B),
\end{aligned}
$$

where $\varsigma=1 / k^{2}$. For the analytic solutions near the point $\varsigma=0$, the Taylor series is $A=\sum_{l=1}^{\infty} \alpha_{l} \varsigma^{l}, B=\sum_{l=1}^{\infty} \beta_{l} \varsigma^{l}$, where $\alpha_{1}=-\frac{4}{9}, \beta_{1}=-\frac{4}{5}, \alpha_{2}=\frac{80}{2187}, \beta_{2}=\frac{4}{27}, \ldots$ The first term gives for the frequency (3.21) the same limit,

$$
\omega_{ \pm}=-\frac{2}{9} \pm i|k| \sqrt{3}
$$

and the higher terms give some corrections.

Let us match the Navier-Stokes term and the first term in the $1 / k^{2}$ expansion. We get

$$
A \approx-\frac{4}{3+9 k^{2}}, \quad B \approx-\frac{4}{3+5 k^{2}}
$$

and

$$
\sigma_{k}=i k A\left(k^{2}\right) u_{k}-k^{2} B\left(k^{2}\right) p_{k} \approx-\frac{4 i k}{3+9 k^{2}} u_{k}+\frac{4 k^{2}}{3+5 k^{2}} p_{k} .
$$

This simplest nonlocality captures the main effects: the asymptotics for short waves (large $k^{2}$ ) and the Navier-Stokes approximation for hydrodynamics for smooth solutions with bounded derivatives and small Knudsen and Mach numbers $\left(\right.$ small $\left.k^{2}\right)$.

The saturation of dissipation at large $k^{2}$ is a universal effect, and hydrodynamics that do not take this effect into account cannot pretend to be a universal asymptotic equation.

This section demonstrates that for the simple kinetic model (1.6):

- The Chapman-Enskog series amounts to an algebraic invariant manifold, and the "smallness" of the Knudsen number $\epsilon$ used to develop the Chapman-Enskog procedure is no longer necessary.

- The exact dispersion relation (3.24) on the algebraic invariant manifold is stable for all wave lengths. 
- The exact result of the Chapman-Enskog procedure has a clear nonpolynomial character. The resulting exact hydrodynamics are essentially nonlocal in space. For this reason, even if the hydrodynamic equations of a certain level of the approximation are stable, they cannot reproduce the nonpolynomial behavior for sufficiently short waves.

- The Newton iterations for the invariance equations provide much better results than the Chapman-Enskog expansion. The first iteration gives the Navier-Stokes asymptotics for long waves and the qualitatively correct behavior with saturation for short waves. The second iteration gives the proper higher-order approximation in the long wave limit and the quantitatively proper asymptotic for short waves.

In the next section we extend these results to a general linear kinetic equation.

\section{Algebraic invariant manifold \\ FOR GENERAL LINEAR KINETICS IN ONE DIMENSION}

\subsection{General form of the invariance equation for one-dimensional linear}

kinetics. For linearized kinetic equations, it is convenient to start directly with the Fourier transformed system.

Let us consider two sets of variables: macroscopic variables $M$ and microscopic variables $\mu$. The corresponding vector spaces are $E_{M}\left(M \in E_{M}\right)$ and $E_{\mu}\left(\mu \in E_{\mu}\right)$, $k$ is the wave vector, and the initial kinetic system in the Fourier space for functions $M_{k}(t)$ and $\mu_{k}(t)$ has the following form:

$$
\begin{aligned}
\partial_{t} M_{k} & =i k L_{M M} M_{k}+i k L_{M \mu} \mu_{k}, \\
\partial_{t} \mu_{k} & =i k L_{\mu M} M_{k}+i k L_{\mu \mu} \mu_{k}+C \mu_{k},
\end{aligned}
$$

where $L_{M M}: E_{M} \rightarrow E_{M}, L_{M \mu} E_{\mu} \rightarrow E_{M}, L_{\mu M}: E_{M} \rightarrow E_{\mu}, L_{\mu \mu}: E_{\mu} \rightarrow E_{\mu}$, and $C: E_{\mu} \rightarrow E_{\mu}$ are constant linear operators (matrices).

The only requirement for the following algebra is the operator $C: E_{\mu} \rightarrow E_{\mu}$ is invertible. (Of course, for further properties such as stability of reduced equations, we need more assumptions such as stability of the whole system (4.1) and negative definiteness of $C$.)

We look for a hydrodynamic invariant manifold in the form

$$
\mu_{k}=\mathcal{X}(k) M_{k}
$$

where $\mathcal{X}(k): E_{M} \rightarrow E_{\mu}$ is a linear map for all $k$.

The corresponding exact hydrodynamic equation is

$$
\partial_{t} M_{k}=i k\left[L_{M M}+L_{M \mu} \mathcal{X}(k)\right] M_{k} .
$$

Calculate the micro- and macroscopic derivatives of $\mu_{k}$ (4.2) exactly as in (3.14) and (3.15):

$$
\begin{aligned}
\partial_{t}^{\text {micro }} \mu_{k} & =\left[i k L_{\mu M}+i k L_{\mu \mu} \mathcal{X}(k)+C \mathcal{X}(k)\right] M_{k}, \\
\partial_{t}^{\text {macro }} \mu_{k} & =\left[i k \mathcal{X}(k) L_{M M}+i k \mathcal{X}(k) L_{M \mu} \mathcal{X}(k)\right] M_{k} .
\end{aligned}
$$

The invariance equation for $\mathcal{X}(k)$ is again a system of algebraic equations (a quadratic matrix equation):

$$
\mathcal{X}(k)=i k C^{-1}\left[-L_{\mu M}+\left(\mathcal{X}(k) L_{M M}-L_{\mu \mu} \mathcal{X}(k)\right)+\mathcal{X}(k) L_{M \mu} \mathcal{X}(k)\right] .
$$


This is a general invariance equation for linear kinetic systems (4.1). The ChapmanEnskog series is a Taylor expansion for the solution of this equation at $k=0$. Thus, immediately we get the first terms:

$$
\mathcal{X}(0)=0, \mathcal{X}^{\prime}(0)=-i C^{-1} L_{\mu M}, \mathcal{X}^{\prime \prime}(0)=2 C^{-1}\left(C^{-1} L_{\mu M} L_{M M}-L_{\mu \mu} C^{-1} L_{\mu M}\right) .
$$

The sequence of the Euler, Navier-Stokes, and Burnett approximations is

$$
\begin{aligned}
\partial_{t} M_{k}= & i k L_{M M} M_{k} \text { (Euler), } \\
\partial_{t} M_{k}= & i k L_{M M} M_{k}+k^{2} L_{M \mu} C^{-1} L_{\mu M} M_{k} \text { (Navier-Stokes), } \\
\partial_{t} M_{k}= & i k L_{M M} M_{k}+k^{2} L_{M \mu} C^{-1} L_{\mu M} M_{k} \\
& \quad+i k^{3} L_{M \mu} C^{-1}\left(C^{-1} L_{\mu M} L_{M M}-L_{\mu \mu} C^{-1} L_{\mu M}\right) M_{k} \text { (Burnett). }
\end{aligned}
$$

Let us use the identity $\mathcal{X}(0)=0$ and the fact that the functions in the $x$-space are real valued. We can separate odd and even parts of $\mathcal{X}(k)$ and write

$$
\mathcal{X}(k)=i k \mathcal{A}\left(k^{2}\right)+k^{2} \mathcal{B}\left(k^{2}\right)
$$

where $\mathcal{A}(y)$ and $\mathcal{B}(y)$ are real-valued matrices. For these unknowns, the invariance equation is even closer to the simple example (3.17):

$$
\begin{aligned}
\mathcal{A}\left(k^{2}\right)= & C^{-1}\left[-L_{\mu M}+k^{2}\left(\mathcal{B}\left(k^{2}\right) L_{M M}-L_{\mu \mu} \mathcal{B}\left(k^{2}\right)\right)\right. \\
& \left.-k^{2} \mathcal{A}\left(k^{2}\right) L_{M \mu} \mathcal{A}\left(k^{2}\right)+k^{4} \mathcal{B}\left(k^{2}\right) L_{M \mu} \mathcal{B}\left(k^{2}\right)\right], \\
\mathcal{B}\left(k^{2}\right)= & -C^{-1}\left[\left(\mathcal{A}\left(k^{2}\right) L_{M M}-L_{\mu \mu} \mathcal{A}\left(k^{2}\right)\right)\right. \\
& \left.+k^{2} \mathcal{A}\left(k^{2}\right) L_{M \mu} \mathcal{B}\left(k^{2}\right)+k^{2} \mathcal{B}\left(k^{2}\right) L_{M \mu} \mathcal{A}\left(k^{2}\right)\right] .
\end{aligned}
$$

4.2. Hyperbolicity of exact hydrodynamics. Hyperbolicity is an important property of the exact hydrodynamics. Let us recall that the linear system represented in Fourier space by the equation

$$
\partial_{t} u_{k}=-i A(k) u_{k}
$$

is hyperbolic if for every $t \geq 0$ the operator $\exp (-i t A(k))$ is uniformly bounded as a function of $k$ (it is sufficient to take $t=1$ ). This means that the Cauchy problem for this system is well-posed forward in time.

This system is strongly hyperbolic if for every $t \in \mathbb{R}$ the operator $\exp (-i t A(k))$ is uniformly bounded as a function of $k$ (it is sufficient to take $t= \pm 1$ ). This means that the Cauchy problem for this system is well-posed both forward and backward in time.

Proposition 4.1 (Preservation of hyperbolicity). Let the original system (4.1) be (strongly) hyperbolic. Then the reduced system (4.3) is also (strongly) hyperbolic if the lifting operator $\mathcal{X}(k)$ (4.2) is a bounded function of $k$.

Proof. Hyperbolicity (strong hyperbolicity) is just a requirement of the uniform boundedness in $k$ of the solutions of (4.1) for each $t>0$ (or for all $t$ ) with uniformly bounded in $k$ initial conditions. For the exact hydrodynamics, solutions of the projected equations are projections of the solutions of the original system. Let the original system (4.1) be (strongly) hyperbolic. If the lifting operator $\mathcal{X}(k)$ is a bounded function of $k$, then for the uniformly bounded initial condition $M_{k}$ the corresponding initial value $\mu_{k}=\mathcal{X}(k) M_{k}$ is also bounded and, due to the hyperbolicity of (4.1), the projection of the solution is uniformly bounded in $k$ for all $t \geq 0$. In the following commutative diagram, the upper horizontal arrow and 
the vertical arrows are the bounded operators; hence, the lower horizontal arrow is also a bounded operator.

$$
\begin{aligned}
& \left(M_{k}(0), \mu_{k}(0)\right) \stackrel{\text { Time shift (initial eq.) }}{\longrightarrow} \quad\left(M_{k}(t), \mu_{k}(t)\right) \\
& \text { Lifting } \uparrow \\
& M_{k}(0) \stackrel{\text { Exact hydrodynamics }}{\longrightarrow} M_{k}(t)
\end{aligned}
$$

To analyze the boundedness of the lifting operator, we have to study the asymptotics of the solution of the invariance equation at the infinitely distant point $k^{2}=\infty$. If this is a regular point, then we can find the Taylor expansion in powers of $\varsigma=\frac{1}{k^{2}}, A=\sum_{l} \alpha_{l} \varsigma^{l}$, and $B=\sum_{l} \beta_{l} \varsigma^{l}$. For the boundedness of $\mathcal{X}(k)$ (4.7), we should take in these series $\alpha_{0}=\beta_{0}=0$. If the solution of the invariance equation is a real analytic function for $0 \geq k^{2} \geq \infty$, then the condition is sufficient for the hyperbolicity of the projected equation (4.3). If $\mathcal{X}(k)$ is an exact solution of the algebraic invariance equation (4.5), then the hydrodynamic equation (4.3) gives the exact reduction of (4.1). Various approximations give the approximate reduction like the Chapman-Enskog approximations (4.6).

The expansion near an infinitely distant point is useful but may be not so straightforward. Nevertheless, if such an expansion exists, then we can immediately produce the matched asymptotics.

Thus, as we can see, the summation of the Chapman-Enskog series to an algebraic manifold is not just a coincidence but a typical effect for kinetic equations. For a specific kinetic system we have to make use of all the existing symmetries such as parity and rotation symmetry in order to reduce the dimension of the invariance equation and to select the proper physical solution. Another simple but important condition is that all the kinetic and hydrodynamic variables should be real valued. The third selection rule is the behavior of the spectrum near $k=0$ : the attenuation rate should go to zero when $k \rightarrow 0$.

The Chapman-Enskog expansion is a Taylor series (in $k$ ) for the solution of the invariance equation. In general, there is no reason to believe that the first few terms of the Taylor series at $k=0$ properly describe the asymptotic behavior of the solutions of the invariance equation (4.5) for all $k$. Already the simple examples such as (3.12) reveal that the exact hydrodynamic is essentially nonlocal and the behavior of the attenuation rate at $k \rightarrow \infty$ does not correspond to any truncation of the Chapman-Enskog series.

Of course, for a numerical solution of (4.5), the Taylor series expansion is not the best approach. The Newton method gives much better results, and even the first approximation may be very close to the solution [20.

In the next section we show that for more complex kinetic equations, the situation may be even more involved, and both the truncation and the summation of the whole series may become meaningless for sufficiently large $k$. In these cases, the hydrodynamic solution of the invariance equations does not exist for large $k$, and the whole problem of hydrodynamic reduction has no solution. We will see how the hydrodynamic description is destroyed and the coupling between hydrodynamic and nonhydrodynamic modes becomes permanent and indestructible. Perhaps, the only advice in this situation may be to change the set of variables or to modify the 
projector onto these variables: if hydrodynamics exist, then the set of hydrodynamic variables or the projection on these variables should be different.

4.3. Destruction of hydrodynamic invariant manifold for short waves in moment equations. In this section we study the one-dimensional version of the Grad equations (1.2) and (1.3) in the $k$-representation:

$$
\begin{aligned}
\partial_{t} \rho_{k} & =-i k u_{k}, \\
\partial_{t} u_{k} & =-i k \rho_{k}-i k T_{k}-i k \sigma_{k}, \\
\partial_{t} T_{k} & =-\frac{2}{3} i k u_{k}-\frac{2}{3} i k q_{k}, \\
\partial_{t} \sigma_{k} & =-\frac{4}{3} i k u_{k}-\frac{8}{15} i k q_{k}-\sigma_{k}, \\
\partial_{t} q_{k} & =-\frac{5}{2} i k T_{k}-i k \sigma_{k}-\frac{2}{3} q_{k} .
\end{aligned}
$$

The Grad system (4.10) provides the simplest coupling of the hydrodynamic variables $\rho_{k}, u_{k}$, and $T_{k}$ to the nonhydrodynamic variables, $\sigma_{k}$ and $q_{k}$, the latter is the heat flux. We need to reduce the Grad system (4.10) to the three hydrodynamic equations with respect to the variables $\rho_{k}, u_{k}$, and $T_{k}$. That is, in the general notations of the previous section, $M=\rho_{k}, u_{k}, T_{k}, \mu=\sigma_{k}, q_{k}$, and we have to express the functions $\sigma_{k}$, and $q_{k}$ in terms of $\rho_{k}, u_{k}$, and $T_{k}$ :

$$
\sigma_{k}=\sigma_{k}\left(\rho_{k}, u_{k}, T_{k}, k\right), \quad q_{k}=q_{k}\left(\rho_{k}, u_{k}, T_{k}, k\right) .
$$

The derivation of the invariance equation for the system (4.10) goes along the same lines as in the previous sections. The quantities $\rho$ and $T$ are scalars, $u$ and $q$ are (one-dimensional) vectors, and the (one-dimensional) stress "tensor" $\sigma$ is again a scalar. The vectors and scalars transform differently under the parity transformation $x \mapsto-x, k \mapsto-k$. We use this symmetry property and find the representation (4.2) of $\sigma, q$ similar to (3.13):

$$
\begin{aligned}
\sigma_{k} & =i k A\left(k^{2}\right) u_{k}-k^{2} B\left(k^{2}\right) \rho_{k}-k^{2} C\left(k^{2}\right) T_{k}, \\
q_{k} & =i k X\left(k^{2}\right) \rho_{k}+i k Y\left(k^{2}\right) T_{k}-k^{2} Z\left(k^{2}\right) u_{k},
\end{aligned}
$$

where the functions $A, \ldots, Z$ are the unknowns in the invariance equation. By the nature of the $\mathrm{CE}$ recurrence procedure for the real-valued in $x$-space kinetic equations, $A, \ldots, Z$ are real-valued functions.

Let us find the microscopic and macroscopic time derivatives (4.4). Computing the microscopic time derivative of the functions (4.11), due to the two last equations of the Grad system (4.10) we derive

$$
\begin{aligned}
\partial_{t}^{\text {micro }} \sigma_{k} & =-i k\left(\frac{4}{3}-\frac{8}{15} k^{2} Z+A\right) u_{k} \\
& +k^{2}\left(\frac{8}{15} X+B\right) \rho_{k}+k^{2}\left(\frac{8}{15} Y+C\right) T_{k} \\
\partial_{t}^{\text {micro }} q_{k} & =k^{2}\left(A+\frac{2}{3} Z\right) u_{k}+i k\left(k^{2} B-\frac{2}{3} X\right) \rho_{k} \\
& -i k\left(\frac{5}{2}-k^{2} C-\frac{2}{3} Y\right) T_{k}
\end{aligned}
$$


On the other hand, computing the macroscopic time derivative of the functions (4.11), due to the first three equations of the system (4.10), we obtain

$$
\begin{aligned}
\partial_{t}^{\text {macro }} \sigma_{k} & =\frac{\partial \sigma_{k}}{\partial u_{k}} \partial_{t} u_{k}+\frac{\partial \sigma_{k}}{\partial \rho_{k}} \partial_{t} \rho+\frac{\partial \sigma_{k}}{\partial T_{k}} \partial_{t} T_{k} \\
& =i k\left(k^{2} A^{2}+k^{2} B+\frac{2}{3} k^{2} C-\frac{2}{3} k^{2} k^{2} C Z\right) u_{k} \\
& +\left(k^{2} A-k^{2} k^{2} A B-\frac{2}{3} k^{2} k^{2} C X\right) \rho_{k} \\
& +\left(k^{2} A-k^{2} k^{2} A C-\frac{2}{3} k^{2} k^{2} C Y\right) T_{k}, \\
\partial_{t}^{\text {macro }} q_{k}= & \frac{\partial q_{k}}{\partial u_{k}} \partial_{t} u_{k}+\frac{\partial q_{k}}{\partial \rho_{k}} \partial_{t} \rho u_{k}+\frac{\partial q_{k}}{\partial T_{k}} \partial_{t} T_{k} \\
= & \left(-k^{2} k^{2} Z A+k^{2} X+\frac{2}{3} k^{2} Y-\frac{2}{3} k^{2} k^{2} Y Z\right) u_{k} \\
& +i k\left(k^{2} Z-k^{2} k^{2} Z B+\frac{2}{3} k^{2} Y X\right) \rho_{k} \\
& +i k\left(k^{2} Z-k^{2} k^{2} Z C+\frac{2}{3} k^{2} Y^{2}\right) T_{k} .
\end{aligned}
$$

The invariance equation (4.5) for this case is a system of six coupled quadratic equations with quadratic in $k^{2}$ coefficients:

$$
\begin{aligned}
& F_{1}=-\frac{4}{3}-A-k^{2}\left(A^{2}+B-\frac{8 Z}{15}+\frac{2 C}{3}\right)+\frac{2}{3} k^{4} C Z=0, \\
& F_{2}=\frac{8}{15} X+B-A+k^{2} A B+\frac{2}{3} k^{2} C X=0, \\
& F_{3}=\frac{8}{15} Y+C-A+k^{2} A C+\frac{2}{3} k^{2} C Y=0, \\
& F_{4}=A+\frac{2}{3} Z+k^{2} Z A-X-\frac{2}{3} Y+\frac{2}{3} k^{2} Y Z=0, \\
& F_{5}=k^{2} B-\frac{2}{3} X-k^{2} Z+k^{4} Z B-\frac{2}{3} Y X=0, \\
& F_{6}=-\frac{5}{2}-\frac{2}{3} Y+k^{2}(C-Z)+k^{4} Z C-\frac{2}{3} k^{2} Y^{2}=0 .
\end{aligned}
$$

There are several approaches to deal with this system. One can easily calculate the Taylor series for $A, B, C, X, Y, Z$ in powers of $k^{2}$ at the point $k=0$. In application to (4.11) this is exactly the Chapman-Enskog series (the Taylor series for $\sigma$ and q). To find the linear and quadratic in $k$ terms in (4.11), we need just a zeroth approximation for $A, B, C, X, Y, Z$ from (4.12):

$$
A=B=-\frac{4}{3}, C=\frac{2}{3}, X=0, Y=-\frac{15}{4}, Z=\frac{7}{4} .
$$

This is the Burnett approximation:

$$
\begin{aligned}
\sigma_{k} & =-\frac{4}{3} i k u_{k}+\frac{4}{3} k^{2} \rho_{k}-\frac{2}{3} k^{2} T_{k} \\
q_{k} & =-\frac{15}{4} i k T_{k}-\frac{7}{4} k^{2} u_{k}
\end{aligned}
$$


The dispersion relation for this Burnett approximation coincides with the one obtained by Bobylëv [9] from the Boltzmann equation for Maxwell molecules, and the short waves are unstable in this approximation.

Direct Newton iterations produce more sensible results. Thus, starting from $A=B=C=X=Y=Z=0$, we get the first iteration

$$
\begin{aligned}
& A_{1}=-20 \frac{141 k^{2}+20}{867 k^{2} k^{2}+2105 k^{2}+300}, \\
& B_{1}=-20 \frac{459 k^{2} k^{2}+810 k^{2}+100}{3468 k^{2} k^{2} k^{2}+12755 k^{2} k^{2}+11725 k^{2}+1500}, \\
& C_{1}=-10 \frac{51 k^{2} k^{2}-485 k^{2}-100}{3468 k^{2} k^{2} k^{2}+12755 k^{2} k^{2}+11725 k^{2}+1500}, \\
& X_{1}=-\frac{375 k^{2}\left(21 k^{2}-5\right)}{2\left(3468 k^{2} k^{2} k^{2}+12755 k^{2} k^{2}+11725 k^{2}+1500\right)}, \\
& Y_{1}=-\frac{225\left(394 k^{2} k^{2}+685 k^{2}+100\right)}{4\left(3468 k^{2} k^{2} k^{2}+12755 k^{2} k^{2}+11725 k^{2}+1500\right)}, \\
& Z_{1}=-15 \frac{153 k^{2}+35}{867 k^{2} k^{2}+2105 k^{2}+300} .
\end{aligned}
$$

The corresponding hydrodynamics are nonlocal but stable and were first obtained by a partial summation (regularization) of the Chapman-Enskog series [49.

A numerical solution of the invariance equation (4.12) is also straightforward and does not produce any serious problem. Selection of the proper (Chapman-Enskog) branch of the solution is set by the asymptotics, $\omega \rightarrow 0$ when $k \rightarrow 0$.

The dispersion equation for frequency $\omega$ is

$$
\begin{aligned}
\omega^{3} & -k^{2}\left(\frac{2}{3} Y+A\right) \omega^{2} \\
& +k^{2}\left(\frac{5}{3}-\frac{2}{3} k^{2} Z-\frac{2}{3} k^{2} C-k^{2} B+\frac{2}{3} k^{2} A Y+\frac{2}{3} k^{2} k^{2} C Z\right) \omega \\
& +\frac{2}{3} k^{2}\left(k^{2} X-k^{2} Y+k^{2} k^{2} B Y-k^{2} k^{2} X C\right)=0 .
\end{aligned}
$$

The real-valued solution to the invariance equation (4.12) does not exist for sufficiently large $k$. (A telling simple example of such a behavior of real algebraic sets gives the equation $k^{2}\left(1-k^{2}\right)+A^{2}=0$.) Above a critical value $k_{c} \approx 0.3023$, the Chapman-Enskog branch in (4.12) disappears and two complex conjugated solutions emerge. This situation becomes clear if we look at the dispersion curves (Figure 4). For $k<k_{c}$, the Chapman-Enskog branch of the dispersion relation consists of three hydrodynamic modes starting from 0 at $k=0$. Two nonhydrodynamic modes start from strictly negative values at $k=0$ and are real-valued. They describe the relaxation to the hydrodynamic invariant manifold from the initial conditions outside this manifold. (This is, in other words, relaxation of the nonhydrodynamic variables, $\sigma_{k}$ and $q_{k}$, to their values $\sigma_{k}\left(\rho_{k}, u_{k}, T_{k}, k\right)$ and $q_{k}\left(\rho_{k}, u_{k}, T_{k}, k\right)$.) For $k<k_{c}$, the nonhydrodynamic modes are real valued, the relaxation goes exponentially, without damped oscillations. At $k=k_{c}$, one root from the nonhydrodynamic branch crosses a real-valued root of the hydrodynamic branch, and they together transform into a couple of complex conjugated roots when $k>k_{c}$. It is impossible to capture two pairs of complex modes by an equation for 


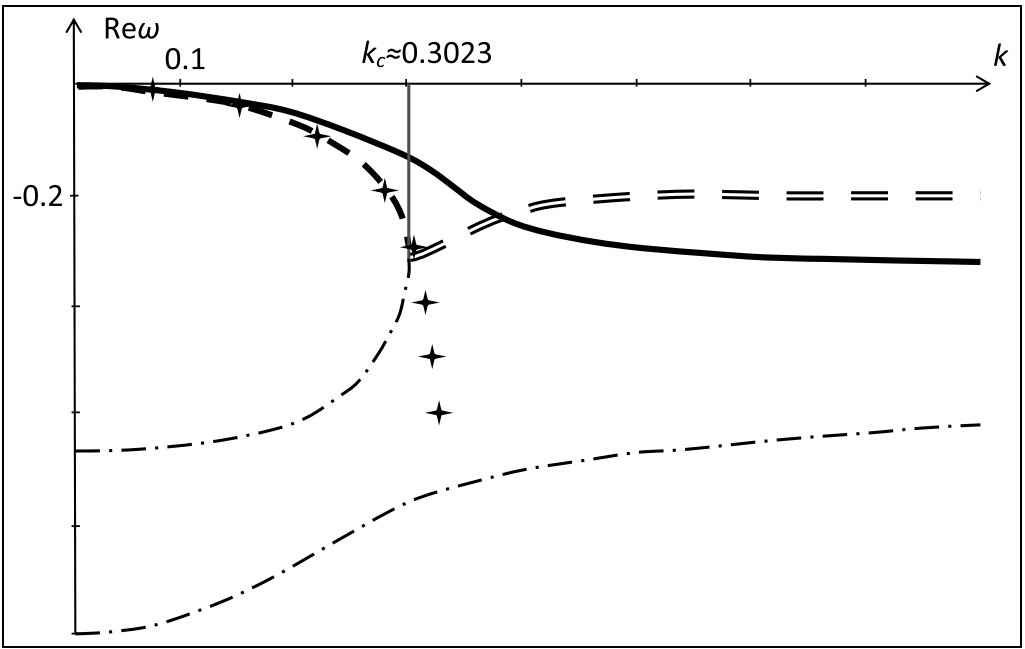

FiguRE 4. The dispersion relation for the linearized onedimensional Grad system (4.10). The solution for the whole kinetic system (4.10) features five $\omega$ 's, while the motions on the hydrodynamic invariant manifold has three $\omega$ 's for each $k<k_{c}$ and destroys for $k \geq k_{c}$. The bold solid line shows the hydrodynamic acoustic mode (two complex conjugated roots). The bold dashed line for $k<k_{c}$ is the hydrodynamic diffusion mode (a real root). At $k=k_{c}$ this line meets a real root of nonhydrodynamic mode (thin dash-dot line) and for $k>k_{c}$, they turn into a couple of complex conjugated roots (bold double-dashed line at $k>k_{c}$ ). The four-point stars correspond to the third Newton iteration for the diffusion mode. A dash-and-dot line at the bottom of the plot shows the isolated nonhydrodynamic mode (single real root of $(2.18)$ ).

three macroscopic variables, and at the same time it is impossible to separate two complex conjugated modes between two systems of real-valued equations.

For small $k$, when the separation of time between the "fast" collision term and the "not-so-fast" advection is significant, there is an essential difference between the relaxation of hydrodynamic and nonhydrodynamic variables: $\rho$ and $u$ do not change in collision and their relaxation is relatively slow, but $\sigma$ and $q$ are directly affected by collisions and their relaxation to $\sigma_{k}\left(\rho_{k}, u_{k}, T_{k}, k\right)$ and $q_{k}\left(\rho_{k}, u_{k}, T_{k}, k\right)$ is fast. Nevertheless, when $k$ grows and achieves $k_{c}$, the difference between the hydrodynamic and nonhydrodynamic variables becomes less pronounced. In such a case, the four-dimensional invariant manifold may describe the relaxation better. For this purpose, we can create the invariance equation for an extended list of four "hydrodynamic variables" and repeat the construction. Instead of the selection of the Chapman-Enskog branch only, we have to select a continuous branch which includes the roots with $\omega \rightarrow 0$ when $k \rightarrow 0$.

The two-dimensional algebraic manifold given by the dispersion equation (4.14) and the invariance equation (4.12) represents the important properties of the hydrodynamic invariant manifold (see Figure 4). In particular, the crucial question is 
the existence of the Chapman-Enskog branch and the description of the connected component of this curve which includes the germ of the Chapman-Enskog branch near $k=0$.

Iterations of the Newton method for the invariance equation converge fast to the solution with singularity. For $k<k_{c}$ the corresponding attenuation rates converge to the exact solution and for $k>k_{c}$ the real part of the diffusion mode Re $\omega \rightarrow-\infty$ with Newton's iterations (Figure 4). The corresponding limit system has the infinitely fast decay of the diffusion mode when $k>k_{c}$. This regularization of singularities by infinite dissipation is quite typical for the application of the Newton method to the solution of the invariance equation. The "solid jet" limit for the extremely fast compressions gives us another example [55] (see also Section 5.2).

4.4. Invariant manifolds, entanglement of hydrodynamic and nonhydrodynamic modes, and saturation of dissipation for the three-dimensional 13-moment Grad system. The 13-moment linear Grad system consists of 13 linearized PDE's (1.2), (1.3) giving the time evolution of the hydrodynamic fields (density $\rho$, velocity vector field $\boldsymbol{u}$, and temperature $T$ ) and of higher-order distinguished moments: five components of the symmetric traceless stress tensor $\boldsymbol{\sigma}$ and three components of the heat flux $\boldsymbol{q}[68$. With this example, we conclude the presentation of exact hydrodynamic manifolds for linearized Grad models.

A point of departure is the Fourier transform of the linearized three-dimensional 13-moment Grad system:

$$
\begin{aligned}
\partial_{t} \rho_{k} & =-i \boldsymbol{k} \cdot \boldsymbol{u}_{k}, \\
\partial_{t} \boldsymbol{u}_{k} & =-i \boldsymbol{k} \rho_{k}-i \boldsymbol{k} T_{k}-i \boldsymbol{k} \cdot \boldsymbol{\sigma}_{k}, \\
\partial_{t} T_{k} & =-\frac{2}{3} i \boldsymbol{k} \cdot\left(\boldsymbol{u}_{k}+\boldsymbol{q}_{k}\right), \\
\partial_{t} \boldsymbol{\sigma}_{k} & =-2 i \overline{\boldsymbol{k} \boldsymbol{u}_{k}}-\frac{4}{5} i \overline{\boldsymbol{k q}}-\boldsymbol{\sigma}_{k}, \\
\partial_{t} \boldsymbol{q}_{k} & =-\frac{5}{2} i \boldsymbol{k} T_{k}-i \boldsymbol{k} \cdot \boldsymbol{\sigma}_{k}-\frac{2}{3} \boldsymbol{q},
\end{aligned}
$$

where $\boldsymbol{k}$ is the wave vector; $\rho_{k}, \boldsymbol{u}_{k}$ and $T_{k}$ are the Fourier images for density, velocity, and temperature, respectively; and $\boldsymbol{\sigma}_{k}$ and $\boldsymbol{q}_{k}$ are the nonequilibrium traceless symmetric stress tensor $(\overline{\boldsymbol{\sigma}}=\boldsymbol{\sigma})$ and heat flux vector components, respectively.

Decompose the vectors and tensors into the parallel (longitudinal) and orthogonal (lateral) parts with respect to the wave vector $\boldsymbol{k}$, because the fields are rotationally symmetric around any chosen direction $\boldsymbol{k}$. A unit vector in the direction of the wave vector is $\boldsymbol{e}=\boldsymbol{k} / k, k=|\boldsymbol{k}|$, and the corresponding decomposition is $\boldsymbol{u}_{k}=u_{k}^{\|} \boldsymbol{e}+\boldsymbol{u}_{k}^{\perp}, \boldsymbol{q}_{k}=q_{k}^{\|} \boldsymbol{e}+\boldsymbol{q}_{k}^{\perp}$, and $\boldsymbol{\sigma}_{k}=\frac{3}{2} \sigma_{k}^{\|} \overline{\boldsymbol{e} \boldsymbol{e}}+2 \boldsymbol{\sigma}_{k}^{\perp}$, where $\boldsymbol{e} \cdot \boldsymbol{u}_{k}^{\perp}=0$, $\boldsymbol{e} \cdot \boldsymbol{q}_{k}^{\perp}=0$, and $\boldsymbol{e} \boldsymbol{e}: \boldsymbol{\sigma}_{k}^{\perp}=0$.

In these variables, the linearized three-dimensional 13-moment Grad system decomposes into two closed sets of equations: one for the longitudinal and another for the lateral modes. The equations for $\rho_{k}, u_{k}^{\|}, T_{k}, \sigma_{k}^{\|}$, and $q_{k}^{\|}$coincide with the one-dimensional Grad system (4.10) from the previous section (the difference is just 


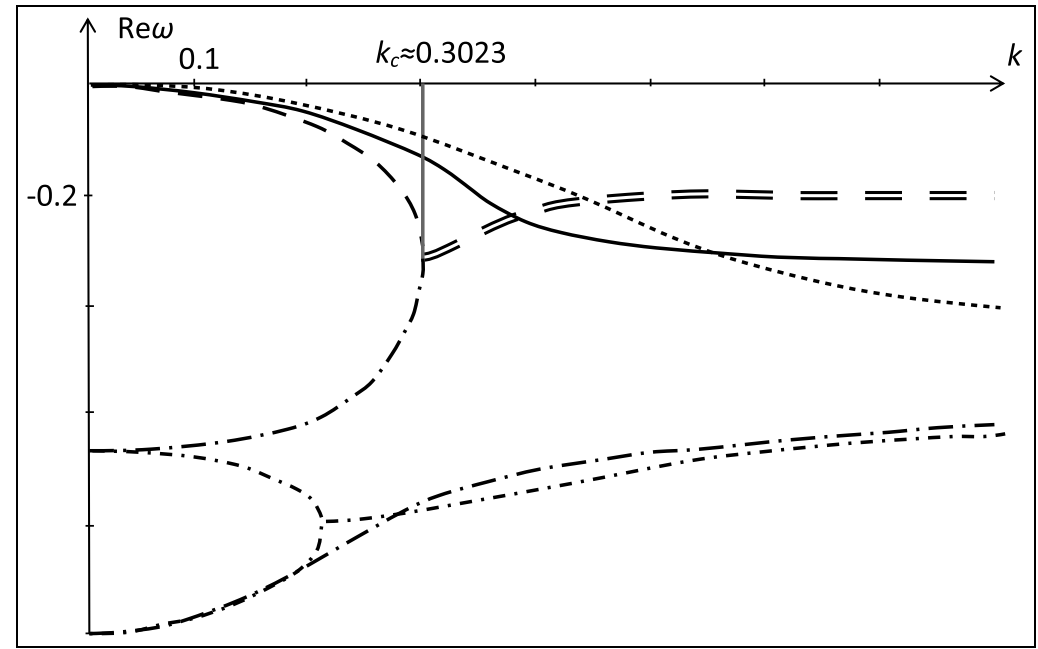

FiguRE 5. The dispersion relation for the linearized threedimensional 13-moment Grad system (1.2), (1.3). The bold solid line shows the hydrodynamic acoustic mode (two complex conjugated roots). The bold dotted line represents the shear mode (double degenerated real-valued root). The bold dashed line for $k<k_{c}$ is the hydrodynamic diffusion mode (a real-valued root). At $k=k_{c}$ this line meets a real-valued root of nonhydrodynamic mode (thin dash-and-dot line), and for $k>k_{c}$ they turn into a couple of complex conjugated roots (bold double-dashed line at $k>k_{c}$ ). Dash-and-dot lines at the bottom of the plot show the separated nonhydrodynamic modes. All the modes demonstrate the saturation of dissipation.

in the superscript $\left.{ }^{\|}\right)$. For the lateral modes we get

$$
\begin{aligned}
& \partial_{t} \boldsymbol{u}_{k}^{\perp}=-i k \boldsymbol{e} \cdot \boldsymbol{\sigma}_{k}^{\perp} \\
& \partial_{t} \boldsymbol{\sigma}_{k}^{\perp}=-i k \overline{\boldsymbol{e} \boldsymbol{u}_{k}^{\perp}}-\frac{2}{5} i k \overline{\boldsymbol{e} \boldsymbol{q}_{k}^{\perp}}-\boldsymbol{\sigma}_{k}^{\perp}, \\
& \partial_{t} \boldsymbol{q}_{k}^{\perp}=-i k \boldsymbol{e} \cdot \boldsymbol{\sigma}_{k}^{\perp}-\frac{2}{3} \boldsymbol{q}_{k}^{\perp} .
\end{aligned}
$$

The hydrodynamic invariant manifold for these decoupled systems is a direct product of the invariant manifolds for (4.10) and for (4.15). The parametrization (4.11), the invariance equation (4.12), the dispersion equation for exact hydrodynamics (4.14), and the plots of the attenuation rates (Figure 4) for (4.10) are presented in the previous section.

For the lateral modes the hydrodynamic variables consist of the two-dimensional vector $\boldsymbol{u}_{k}^{\perp}$. We use the general expression (4.7) and take into account the rotational symmetry for the parametrization of the nonhydrodynamic variables $\boldsymbol{\sigma}_{k}^{\perp}$ and $\boldsymbol{q}_{k}^{\perp}$ by the hydrodynamic ones:

$$
\boldsymbol{\sigma}_{k}^{\perp}=i k D\left(k^{2}\right) \overline{\boldsymbol{e} \boldsymbol{u}_{k}^{\perp}}, \quad \boldsymbol{q}_{k}^{\perp}=-k^{2} U\left(k^{2}\right) \boldsymbol{u}_{k}^{\perp} .
$$


There are two unknown scalar real-valued functions here: $D\left(k^{2}\right)$ and $U\left(k^{2}\right)$. We equate the microscopic and macroscopic time derivatives of the nonhydrodynamic variables and get the invariance conditions:

$$
\begin{aligned}
& \frac{\partial \boldsymbol{\sigma}_{k}^{\perp}}{\partial \boldsymbol{u}_{k}^{\perp}} \cdot\left(-i k \boldsymbol{e} \cdot \boldsymbol{\sigma}_{k}^{\perp}\right)=-i k \overline{\boldsymbol{e} \boldsymbol{u}_{k}^{\perp}}-\frac{2}{5} i k \overline{\boldsymbol{e} \boldsymbol{q}_{k}^{\perp}}-\boldsymbol{\sigma}_{k}^{\perp}, \\
& \frac{\partial \boldsymbol{q}_{k}^{\perp}}{\partial \boldsymbol{u}_{k}^{\perp}} \cdot\left(-i k \boldsymbol{e} \cdot \boldsymbol{\sigma}_{k}^{\perp}\right)=-i k \boldsymbol{e} \cdot \boldsymbol{\sigma}_{k}^{\perp}-\frac{2}{3} \boldsymbol{q}_{k}^{\perp} .
\end{aligned}
$$

We substitute here $\boldsymbol{\sigma}_{k}^{\perp}$ and $\boldsymbol{q}_{k}^{\perp}$ by the expressions (4.16) and derive the algebraic invariance equation for $D$ and $U$, which can be transformed into the form

$$
\begin{aligned}
& 15 k^{4} D^{3}+25 k^{2} D^{2}+\left(10+21 k^{2}\right) D+10=0, \\
& U=-\frac{3 D}{2+3 k^{2} D} .
\end{aligned}
$$

The solution of the cubic equation (4.18) with the additional condition $D(0)=-1$ matches the Navier-Stokes asymptotics and is real valued for all $k^{2}$ [20]. The dispersion equation gives twice-degenerated real-valued shear mode. All 13 modes for the three-dimensional 13-moment linearized Grad system are presented in Figure 5 with five hydrodynamic and eight nonhydrodynamic modes. This plot includes also eight modes (three hydrodynamic and five nonhydrodynamic ones) for the one-dimensional system (4.10). Entanglement between hydrodynamic and nonhydrodynamics modes appears at the same critical value of $k \approx 0.3023$, and the exact hydrodynamics does not exist for larger $k$.

\subsection{Algebraic hydrodynamic invariant manifold for the linearized Boltz-} mann and BGK equations: separation of hydrodynamic and nonhydrodynamic modes. The entanglement of the hydrodynamic and nonhydrodynamic modes at large wave vectors $k$ destroys the exact hydrodynamic for the Grad moment equations. We conjecture that this is the catastrophe of the applicability of the moment equations, and the hydrodynamic manifolds are destroyed together with the Grad approximation. It is plausible that if the linearized collision operator has a spectral gap (see a review in [118]) between the five-time degenerated zero and other eigenvalues, then the algebraic hydrodynamic invariant manifold exists for all $k$. This remains an open question but the numerical calculations of the hydrodynamic invariant manifold available for the linearized kinetic equation (1.1) with the BGK collision operator [7,54] support this conjecture [87.

The incompressible hydrodynamic limit for the scaled solutions of the BGK equation was proven in 2003 [125].

The linearized kinetic equation (1.1) has the form

$$
\partial_{t} f+\boldsymbol{v} \cdot \nabla_{x} f=L f
$$

where $f(t, \boldsymbol{v}, \boldsymbol{x})$ is the deviation of the distribution function from its equilibrium value $f^{*}(\boldsymbol{v}), L$ is the linearized kinetic operator. Operator $L$ is symmetric with respect to the entropic inner product

$$
\langle\varphi, \psi\rangle_{f^{*}}=\int \frac{\varphi(\boldsymbol{v}) \psi(\boldsymbol{v})}{f^{*}(\boldsymbol{v})} \mathrm{d}^{3} \boldsymbol{v} .
$$


In the $L_{2}$ space with this inner product, $\operatorname{ker} L=(\operatorname{im} L)^{\perp}$ is a finite-dimensional subspace. It is spanned by five functions:

$$
f^{*}(\boldsymbol{v}), \boldsymbol{v} f^{*}(\boldsymbol{v}), v^{2} f^{*}(\boldsymbol{v}) .
$$

The hydrodynamic variables (for the given $t$ and $x$ ) are the inner products of these functions on $f(t, \boldsymbol{x}, \boldsymbol{v})$, but it is more convenient to use the orthonormal basis with respect to the product $\langle\cdot, \cdot\rangle_{f *}, \varphi_{1}(\boldsymbol{v}), \ldots, \varphi_{5}(\boldsymbol{v})$. The macroscopic variables are $M_{i}=\left\langle\varphi_{i}, f\right\rangle_{f^{*}}(i=1,2, \ldots, 5)$.

It is convenient to represent $f$ in the form of the direct sum of the macroscopic and microscopic components

$$
f=P_{\text {macro }} f+P_{\text {micro }} f,
$$

where

$$
P_{\text {macro }} f=\sum_{i} \varphi_{i}\left\langle\varphi_{i}, f\right\rangle_{f^{*}}, \quad P_{\text {micro }} f=\left(f-\sum_{i} \varphi_{i}\left\langle\varphi_{i}, f\right\rangle_{f^{*}}\right) .
$$

After the Fourier transformation the linearized kinetic equation is

$$
\partial_{t} f_{k}=-i(\boldsymbol{k}, \boldsymbol{v}) f_{k}+L f_{k} .
$$

The lifting operation $\mathcal{X}(\boldsymbol{k}): M_{k} \mapsto f_{k}$ (4.2) should have the form

$$
\mathcal{X}(\boldsymbol{k})(M)=\sum_{i} M_{i k} \varphi_{i}(\boldsymbol{v})+\sum_{i} M_{i k} \psi_{i}(\boldsymbol{k}, \boldsymbol{v}),
$$

where $\left\langle\varphi_{i}, \psi_{j}\right\rangle_{f^{*}}=0$ for all $i, j=1,2, \ldots, 5$. We equate the microscopic and macroscopic time derivatives (4.4) of $f$ and get the invariance equation (4.5):

$$
\begin{aligned}
L \psi_{j}= & i \boldsymbol{k} \cdot\left[P_{\text {micro }}\left(\boldsymbol{v} \varphi_{j}\right)+P_{\text {micro }}\left(\boldsymbol{v} \psi_{j}\right)\right. \\
& \left.-\sum_{l} \psi_{l}\left\langle\varphi_{l}, \boldsymbol{v} \varphi_{j}\right\rangle_{f^{*}}-\sum_{l} \psi_{l}\left\langle\varphi_{l}, \boldsymbol{v} \psi_{j}\right\rangle_{f^{*}}\right] .
\end{aligned}
$$

For the solution of this equation, it is important that $\operatorname{im} L=\operatorname{im} P_{\text {micro }}$ and the both operators $L$ and $L^{-1}$ are defined and bounded on this microscopic subspace. The linearized BGK collision integral is simply $L=-P_{\text {micro }}$ (the relaxation parameter $\epsilon=1$ ) and the invariance equation has in this case an especially simple form.

In [87] the form of this equation has been analyzed further, and it has been solved numerically by several methods: the Newton iterations and continuation in parameter $k$. The attenuation rates for the Chapman-Enskog branch have been analyzed. All the methods have produced the same results:

(i) the real-valued hydrodynamic invariant manifold exists for all range of $k$, from zero to large values;

(ii) hydrodynamic modes are always separated from the nonhydrodynamic modes (no entanglement effects); and

(iii) the saturation of dissipation exists for large $k$.

\section{HYDRODYNAMIC INVARIANT MANIFOLDS FOR NONLINEAR KINETICS}

5.1. One-dimensional nonlinear Grad equation and nonlinear viscosity. In the preceding sections we represented the hydrodynamic invariant manifolds for linear kinetic equations. The algebraic equations for these manifolds in $k$-space have a relatively simple closed form and can be studied both analytically and numerically. For nonlinear kinetics, the situation is more difficult for a simple reason: it is impossible to cast the problem of the invariant manifold in the form of a system 
of decoupled finite-dimensional problems by the Fourier transform. The equations for the invariant manifolds for the finite-dimensional nonlinear dynamics have been published by Lyapunov in 1892 [11], but even for ODEs this is a nonlinear and rather nonstandard system of PDEs.

There are several ways to study the hydrodynamic invariant manifolds for nonlinear kinetics. In addition to the classical Chapman-Enskog series expansion, we can solve the invariance equation numerically or semi-analytically, for example, by the iterations instead of the power series. In the next section, we demonstrate this method for the Boltzmann equation. In this section, we follow the strategy that seems to be promising: to evaluate the asymptotics of the hydrodynamic invariant manifolds at large gradients and frequencies, and to match these asymptotics with the first Chapman-Enskog terms. For this purpose, we use exact summation of the "leading terms" in the Chapman-Enskog series.

The starting point is the set of the one-dimensional nonlinear Grad equations for the hydrodynamic variables $\rho, u$ and $T$, coupled with the nonhydrodynamic variable $\sigma$, where $\sigma$ is the $x x$-component of the stress tensor:

$$
\begin{aligned}
\partial_{t} \rho & =-\partial_{x}(\rho u) \\
\partial_{t} u & =-u \partial_{x} u-\rho^{-1} \partial_{x} p-\rho^{-1} \partial_{x} \sigma \\
\partial_{t} T & =-u \partial_{x} T-(2 / 3) T \partial_{x} u-(2 / 3) \rho^{-1} \sigma \partial_{x} u \\
\partial_{t} \sigma & =-u \partial_{x} \sigma-(4 / 3) p \partial_{x} u-(7 / 3) \sigma \partial_{x} u-\frac{p}{\mu(T)} \sigma .
\end{aligned}
$$

Here $p=\rho T$ and $\mu(T)$ is the temperature-dependent viscosity coefficient. We adopt the form $\mu(T)=\alpha T^{\gamma}$, where $\gamma$ varies from $\gamma=1$ (Maxwell's molecules) to $\gamma=1 / 2$ (hard spheres) [24.

Our goal is to compute the correction to the Navier-Stokes approximation of the hydrodynamic invariant manifold, $\sigma_{\mathrm{NS}}=-(4 / 3) \mu \partial_{x} u$, for high values of the velocity. Let us consider first the Burnett correction from (5.1)-(5.4):

$$
\sigma_{\mathrm{B}}=-\frac{4}{3} \mu \partial_{x} u+\frac{8(2-\gamma)}{9} \mu^{2} p^{-1}\left(\partial_{x} u\right)^{2}-\frac{4}{3} \mu^{2} p^{-1} \partial_{x}\left(\rho^{-1} \partial_{x} p\right) .
$$

Each further $n$th term of the Chapman-Enskog expansion contributes, among others, a nonlinear term proportional to $\left(\partial_{x} u\right)^{n+1}$. Such terms can be named the high-speed terms since they dominate the rest of the contributions in each order of the Chapman-Enskog expansion when the characteristic average velocity is comparable to the thermal speed. Indeed, let $U$ be the characteristic velocity (the Mach number). Consider the scaling $u=U \widetilde{u}$, where $\widetilde{u}=O(1)$. This velocity scaling is instrumental to the selection of the leading large gradient terms, and the result below is manifestly Galilean-invariant.

The term $\left(\partial_{x} u\right)^{n+1}$ includes the factor $U^{n+1}$, which is the highest possible order of $U$ among the terms available in the $n$th order of the Chapman-Enskog expansion. Simple dimensional analysis leads to the conclusion that such terms are of the form

$$
\mu\left(p^{-1} \mu \partial_{x} u\right)^{n} \partial_{x} u=\mu g^{n} \partial_{x} u,
$$

where $g=p^{-1} \mu \partial_{x} u$ is dimensionless. Therefore, the Chapman-Enskog expansion for the function $\sigma$ may be formally rewritten as

$$
\sigma=-\mu\left\{\frac{4}{3}-\frac{8(2-\gamma)}{9} g+r_{2} g^{2}+\cdots+r_{n} g^{n}+\cdots\right\} \partial_{x} u+\cdots .
$$


The series in the brackets is the collection of the high-speed contributions of interest, coming from all orders of the Chapman-Enskog expansion, while the dots outside the brackets stand for the terms of other natures. Thus after summation the series of the high-speed corrections to the Navier-Stokes approximation for the Grad equations (5.1) takes the form

$$
\sigma_{\mathrm{nl}}=-\mu R(g) \partial_{x} u
$$

where $R(g)$ is a yet unknown function represented by a formal subsequence of Chapman-Enskog terms in the expansion (5.6). The function $R$ can be considered as a dynamic modification of the viscosity $\mu$ due to the gradient of the average velocity.

Let us write the invariance equation for the representation (5.7). We first compute the microscopic derivative of the function $\sigma_{\mathrm{nl}}$ by substituting (5.7) into the right-hand side of (5.4):

$$
\begin{aligned}
\partial_{t}^{\text {micro }} \sigma_{\mathrm{nl}} & =-u \partial_{x} \sigma_{\mathrm{nl}}-\frac{4}{3} p \partial_{x} u-\frac{7}{3} \sigma_{\mathrm{nl}} \partial_{x} u-\frac{p}{\mu(T)} \sigma_{\mathrm{nl}} \\
& =\left\{-\frac{4}{3}+\frac{7}{3} g R+R\right\} p \partial_{x} u+\cdots,
\end{aligned}
$$

where the dots denote the terms irrelevant to the high-speed approximation (5.7).

Second, computing the macroscopic derivative of $\sigma_{\mathrm{nl}}$ due to (5.1), (5.2), and (5.3), we obtain

$$
\partial_{t}^{\text {macro }} \sigma_{\mathrm{nl}}=-\left[\partial_{t} \mu(T)\right] R \partial_{x} u-\mu(T) \frac{\mathrm{d} R}{\mathrm{~d} g}\left[\partial_{t} g\right] \partial_{x} u-\mu(T) R \partial_{x}\left[\partial_{t} u\right] .
$$

In the latter expression, the time derivatives of the hydrodynamic variables should be replaced with the right-hand sides of (5.1), (5.2), and (5.3), where, in turn, $\sigma$ should be replaced by $\sigma_{\mathrm{nl}}$ (5.7). We find

$$
\partial_{t}^{\text {macro }} \sigma_{\mathrm{nl}}=\left\{g R+\frac{2}{3}(1-g R) \times\left(\gamma g R+(\gamma-1) g^{2} \frac{\mathrm{d} R}{\mathrm{~d} g}\right)\right\} p \partial_{x} u+\cdots .
$$

Again we omit the terms irrelevant to the analysis of the leading terms.

Equating the relevant terms in (5.8) and (5.10), we obtain the approximate invariance equation for the function $R$ :

$$
(1-\gamma) g^{2}(1-g R) \frac{\mathrm{d} R}{\mathrm{~d} g}+\gamma g^{2} R^{2}+\left[\frac{3}{2}+g(2-\gamma)\right] R-2=0 .
$$

It is approximate because in the microscopic derivative many terms are omitted, and it becomes more accurate when the velocities are multiplied by a large factor. When $g \rightarrow \pm \infty$, then the viscosity factor (5.11) $R \rightarrow 0$.

For Maxwell's molecules $(\gamma=1)$, (5.11) simplifies considerably, and it becomes the algebraic equation

$$
g^{2} R^{2}+\left(\frac{3}{2}+g\right) R-2=0 .
$$

The solution recovers the Navier-Stokes relation in the limit of small $g$, and for an arbitrary $g$ it reads

$$
R_{\mathrm{MM}}=\frac{-3-2 g+3 \sqrt{1+(4 / 3) g+4 g^{2}}}{4 g^{2}} .
$$




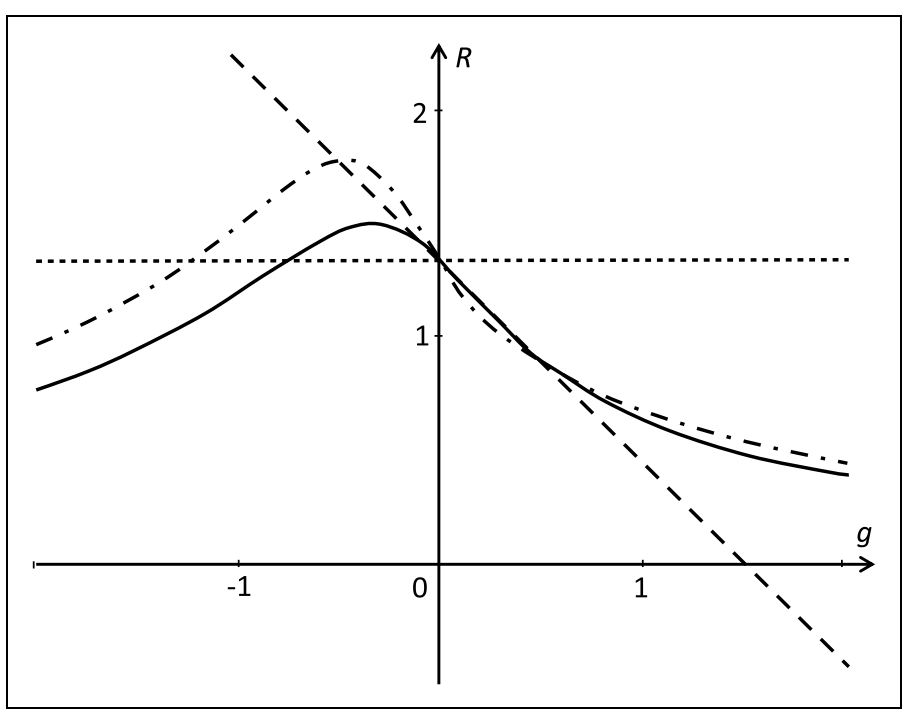

Figure 6. Viscosity factor $R(g)$ (5.11): solid line is $R(g)$ for Maxwell molecules; dash is the Burnett approximation of $R(g)$ for Maxwell molecules; dots are the Navier-Stokes approximation; dash-dots are viscosity factor $R(g)$ for hard spheres, the first approximation (5.14).

The function $R_{\mathrm{MM}}$ (5.13) is plotted in Figure 6, Note that $R_{\mathrm{MM}}$ is positive for all values of its argument $g$, as is appropriate for the viscosity factor, while the Burnett approximation to the function $R_{\mathrm{MM}}$ violates positivity.

For other models $(\gamma \neq 1)$, the invariance equation (5.11) is a nonlinear ODE with the initial condition $R(0)=4 / 3$ (the Navier-Stokes condition). Several ways to derive analytic results are possible. One possibility is to expand the function $R$ into powers of $g$, around the point $g=0$. This brings us back to the original subseries of the Chapman-Enskog expansion (5.6). Instead, we take advantage of the opportunity offered by the parameter $\gamma$. Introduce another parameter $\beta=1-\gamma$, and consider the expansion

$$
R(\beta, g)=R_{0}(g)+\beta R_{1}(g)+\beta^{2} R_{2}(g)+\cdots .
$$

Substituting this expansion into the invariance equation (5.11), we derive $R_{0}(g)=$ $R_{\mathrm{MM}}(g)$,

$$
R_{1}(g)=-g\left(1-g R_{0}\right) \frac{R_{0}+g\left(\mathrm{~d} R_{0} / \mathrm{d} g\right)}{2 g^{2} R_{0}+g+(3 / 2)},
$$

etc. That is, the solution for models different from Maxwell's molecules is constructed in the form of a series with the exact solution for the Maxwell molecules as the leading term. For hard spheres $(\beta=1 / 2)$, the result to the first-order term reads $R_{\mathrm{HS}} \approx R_{\mathrm{MM}}+(1 / 2) R_{1}$. The resulting approximate viscosity factor is shown in Figure 6 (dash-dots line). The features of the approximation obtained are qualitatively the same as in the case of Maxwell molecules.

Precisely the same result for the nonlinear elongational viscosity obtained first from the Grad equations [88] was derived in [44,128] from the solution to the BGK 
kinetic equation in the regime of so-called homo-energetic extension flow. This remarkable fact gives more credit to the derivation of hydrodynamic manifolds from nonlinear Grad equations.

The approximate invariance equation (5.11) defines the relevant physical solution to the viscosity factor for all values of $g$. The hydrodynamic equations are now given by (5.1), (5.2), and (5.3), where $\sigma$ is replaced by $\sigma_{\mathrm{nl}}$ (5.7). First, the correction concerns the nonlinear regime, and, thus, the linearized form of the new equations coincides with the linearized Navier-Stokes equations. Second, the solution (5.13) for Maxwell molecules and the result of the approximation (5.14) for other models and also the numerical solution [90] suggest that the modified viscosity $\mu R$ vanishes in the limit of very high values of the velocity gradients. However, a cautious remark is in order since the original "kinetic" description is Grad's equations (5.1) (5.4) and not the Boltzmann equation. The first Newton iteration for the Boltzmann equation gives a singularity of viscosity at a large negative value of divergency (see below, Section 5.2).

\subsection{Approximate invariant manifold for the Boltzmann equation.}

5.2.1. Invariance equation. We begin with writing down the invariance condition for the hydrodynamic manifold of the Boltzmann equation. A convenient point of departure is the Boltzmann equation (1.1) in a co-moving reference frame,

$$
D_{t} f=-(\boldsymbol{v}-\boldsymbol{u}) \cdot \nabla_{x} f+Q(f),
$$

where $D_{t}$ is the material time derivative, $D_{t}=\partial_{t}+\boldsymbol{u} \cdot \nabla_{x}$. The macroscopic (hydrodynamic) variables are

$$
M=\left\{n ; n \boldsymbol{u} ; \frac{3 n k_{\mathrm{B}} T}{\mu}+n u^{2}\right\}=m[f]=\int\left\{1 ; \boldsymbol{v} ; v^{2}\right\} f \mathrm{~d} \boldsymbol{v},
$$

where $n$ is number density, $\boldsymbol{u}$ is the flow velocity, and $T$ is the temperature; $\mu$ is particle's mass and $k_{\mathrm{B}}$ is Boltzmann's constant. These fields do not change in collisions, hence, the projection of the Boltzmann equation on the hydrodynamic variables is

$$
D_{t} M=-m\left[(\boldsymbol{v}-\boldsymbol{u}) \cdot \nabla_{x} f\right] .
$$

For the given hydrodynamic fields $M$, the local Maxwellian $f_{M}^{\mathrm{LM}}$ (or just $f^{\mathrm{LM}}$ ) is the only zero of the collision integral $Q(f)$.

$$
f^{\mathrm{LM}}=n\left(\frac{2 \pi k_{\mathrm{B}} T}{\mu}\right)^{-3 / 2} \exp \left(-\frac{\mu(\boldsymbol{v}-\boldsymbol{u})^{2}}{2 k_{\mathrm{B}} T}\right) .
$$

The local Maxwellian depends on space through the hydrodynamic fields.

We are looking for the invariant manifold $\boldsymbol{f}_{M}$ in the space of distribution functions parametrized by the hydrodynamic fields. Such a manifold is represented by a lifting map $M \mapsto f_{M}$ that maps the hydrodynamic fields in three-dimensional space, three functions of the space variables, $M=\{n(\boldsymbol{x}), \boldsymbol{u}(\boldsymbol{x}), T(\boldsymbol{x})\}$, into a function of six variables $\boldsymbol{f}_{M}(\boldsymbol{x}, \boldsymbol{v})$. The consistency condition should hold:

$$
m\left[f_{M}\right]=M .
$$

The differential of the lifting operator at the point $M$ is a linear map $\left(D_{M} \boldsymbol{f}_{M}\right)$ : $\delta M \rightarrow \delta f$. 
It is straightforward to write down the invariance condition for the hydrodynamic manifold: The microscopic time derivative of $f_{M}$ is given by the right-hand side of the Boltzmann equation on the manifold

$$
D_{t}^{\text {micro }} f_{M}=-(\boldsymbol{v}-\boldsymbol{u}) \cdot \nabla_{x} f_{M}+Q\left(f_{M}\right),
$$

while the macroscopic time derivative is defined by the chain rule

$$
D_{t}^{\text {macro }} f_{M}=-\left(D_{M} f_{M}\right) m\left[(\boldsymbol{v}-\boldsymbol{u}) \cdot \nabla_{x} f_{M}\right] .
$$

The invariance equation requires that, for any $M$, the outcome of two ways of taking the derivative should be the same:

$$
-\left(D_{M} \boldsymbol{f}_{M}\right) m\left[(\boldsymbol{v}-\boldsymbol{u}) \cdot \nabla_{x} \boldsymbol{f}_{M}\right]=-(\boldsymbol{v}-\boldsymbol{u}) \cdot \nabla_{x} \boldsymbol{f}_{M}+Q\left(\boldsymbol{f}_{M}\right) .
$$

One more field plays a central role in the study of invariant manifolds: the defect of invariance,

$$
\begin{aligned}
\Delta_{M} & =D_{t}^{\text {macro }} \boldsymbol{f}_{M}-D_{t}^{\text {micro }} \boldsymbol{f}_{M} \\
& =-\left(D_{M} \boldsymbol{f}_{M}\right) m\left[(\boldsymbol{v}-\boldsymbol{u}) \cdot \nabla_{x} \boldsymbol{f}_{M}\right]+(\boldsymbol{v}-\boldsymbol{u}) \cdot \nabla_{x} \boldsymbol{f}_{M} .
\end{aligned}
$$

It measures the "noninvariance" of a manifold $\boldsymbol{f}_{M}$.

Let an approximation of the lifting operation $M \rightarrow \boldsymbol{f}_{M}$ be given. The equation of the first iteration for the unknown correction $\delta \boldsymbol{f}_{M}$ of $\boldsymbol{f}_{M}$ is obtained by the linearization (we assume that the initial approximation, $\boldsymbol{f}_{M}$, satisfies the consistency condition and $m[\delta f]=0)$ :

$$
\left(D_{M} \boldsymbol{f}_{M}\right) m\left[(\boldsymbol{v}-\boldsymbol{u}) \cdot \nabla_{x} \delta \boldsymbol{f}_{M}\right]-(\boldsymbol{v}-\boldsymbol{u}) \cdot \nabla_{x} \delta \boldsymbol{f}_{M}+L \delta \boldsymbol{f}_{M}=\Delta_{M}
$$

Here, $L_{M}$ is a linearization of $Q$ at $f_{M}^{\mathrm{LM}}$. If $\boldsymbol{f}_{M}$ is local equilibrium, then the integral operator $L_{M}$ at each point $\boldsymbol{x}$ is symmetric with respect to the entropic inner product (4.20). The equation of iteration (5.21) is linear but with nonconstant in space coefficients because both $\left(D_{M} \boldsymbol{f}_{M}\right)$ and $L_{M}$ depend on $\boldsymbol{x}$.

It is necessary to stress that the standard Newton method does not work in these settings. If $\boldsymbol{f}_{M}$ is not a local equilibria, then $L_{M}$ may be not symmetric and we may lose such instruments as the Fredholm alternative. Therefore, we use in the iterations the linearized operators $L_{M}$ at the local equilibrium and not at the current approximate distribution $\boldsymbol{f}_{M}$ (the Newton-Kantorovich method). We also do not include the differential of the term $\left(D_{M} \boldsymbol{f}_{M}\right) m$ in (5.21). The reason for this incomplete linearization of the invariance equation (5.19) is that it provides convergence to the slowest invariant manifold (at least, for linear vector fields), and other invariant manifolds are unstable in iteration dynamics. The complete linearization does not have this property [53,60].

5.2.2. Invariance correction to the local Maxwellian. Let us choose the local Maxwellian $\boldsymbol{f}_{M}=f^{\mathrm{LM}}$ (5.17) as the initial approximation to the invariant manifold in (5.21). In order to find the right-hand side of this equation, we evaluate the defect of invariance (5.20) $\Delta_{M}=\Delta^{\mathrm{LM}}$ :

$$
\Delta^{\mathrm{LM}}=f^{\mathrm{LM}} D,
$$


where

$$
\begin{aligned}
D= & \left(\frac{\mu(\boldsymbol{v}-\boldsymbol{u})^{2}}{2 k_{\mathrm{B}} T}-\frac{5}{2}\right)(\boldsymbol{v}-\boldsymbol{u}) \cdot \frac{\nabla_{x} T}{T} \\
& +\frac{\mu}{k_{\mathrm{B}} T}\left[(\boldsymbol{v}-\boldsymbol{u}) \otimes(\boldsymbol{v}-\boldsymbol{u})-\frac{1}{3} \mathbf{1}(\boldsymbol{v}-\boldsymbol{u})^{2}\right]: \nabla_{x} \boldsymbol{u}
\end{aligned}
$$

Note that there is no "smallness" parameter involved in the present consideration, the defect of invariance of the local Maxwellian is neither "small" or "large" by itself. We now proceed with finding a correction $\delta f$ to the local Maxwellian on the basis of the linearized equation (5.21) supplemented with the consistency condition,

$$
m[\delta f]=0 .
$$

Note that if we introduce the formal large parameter $L \leftarrow \epsilon^{-1} L$ and look at the leading-order correction $\delta f \leftarrow \epsilon \delta f$, disregarding all the rest in equation (5.21), we get a linear nonhomogeneous integral equation,

$$
\begin{aligned}
\Lambda\left(\delta f / f^{\mathrm{LM}}\right)= & \left(\frac{\mu(\boldsymbol{v}-\boldsymbol{u})^{2}}{2 k_{\mathrm{B}} T}-\frac{5}{2}\right)(\boldsymbol{v}-\boldsymbol{u}) \cdot \frac{\nabla_{x} T}{T} \\
& +\frac{\mu}{k_{\mathrm{B}} T}\left[(\boldsymbol{v}-\boldsymbol{u}) \otimes(\boldsymbol{v}-\boldsymbol{u})-\frac{1}{3} \mathbf{1}(\boldsymbol{v}-\boldsymbol{u})^{2}\right]: \nabla_{x} \boldsymbol{u}
\end{aligned}
$$

where

$$
\Lambda \varphi=\int w\left(\boldsymbol{v}^{\prime}, \boldsymbol{v}_{1}^{\prime} \mid \boldsymbol{v}, \boldsymbol{v}_{1}\right) f^{\mathrm{LM}}\left(\boldsymbol{v}_{1}\right)\left[\varphi\left(\boldsymbol{v}_{1}^{\prime}\right)+\varphi\left(\boldsymbol{v}^{\prime}\right)-\varphi(\boldsymbol{v})-\varphi\left(\boldsymbol{v}_{1}\right)\right] \mathrm{d} \boldsymbol{v}_{1}^{\prime} \mathrm{d} \boldsymbol{v}^{\prime} \mathrm{d} \boldsymbol{v}_{1}
$$

is the linearized Boltzmann collision operator ( $w$ is the scattering kernel; standard notation for the velocities before and after the binary encounter is used). It is readily seen that (5.25) is nothing but the standard equation of the first Chapman-Enskog approximation, whereas the consistency condition (5.24) results in the unique solution (Fredholm alternative) to (5.25). This leads to the classical Navier-Stokes Fourier equations of the Chapman-Enskog method.

Thus, the first iteration (5.21) for the solution of the invariance equation (5.19) with the local Maxwellian as the initial approximation is matched to the first Chapman-Enskog correction to the local Maxwellian. However, equation (5.21) is much more complicated than its Chapman-Enskog limit: equation (5.21) is linear but integro-differential (rather than just the linear integral equation (5.25)), with coefficients varying in space through both $\left(D_{M} f^{\mathrm{LM}}\right)$ and $L$. We shall now describe a microlocal approach for solving (5.21).

5.2.3. Microlocal techniques for the invariance equation. Introducing $\delta f=f^{\mathrm{LM}} \varphi$, equation (5.21) for the local Maxwellian initial approximation can be cast in the form

$$
\Lambda^{*} \varphi-\left(\boldsymbol{V}^{*} \cdot \nabla\right) \varphi=D,
$$

where the enhanced linearized collision integral $\Lambda^{*}$ and the enhanced free flight operator $\left(\boldsymbol{V}^{*} \cdot \nabla\right)$ act as follows. Let us denote $\Pi$ the projection operator $\left(\Pi^{2}=\Pi\right)$,

$$
\Pi g=\left(f^{\mathrm{LM}}\right)^{-1} D_{M} f^{\mathrm{LM}} m\left[f^{\mathrm{LM}} g\right] .
$$


Then in (5.26) we have

$$
\begin{aligned}
\Lambda^{*} \varphi= & \Lambda \varphi+(\Pi-1)(r \varphi), \\
r= & (\boldsymbol{v}-\boldsymbol{u}) \cdot \frac{\nabla_{x} n}{n}+\frac{\mu}{k_{\mathrm{B}} T}(\boldsymbol{v}-\boldsymbol{u}) \otimes(\boldsymbol{v}-\boldsymbol{u}): \nabla_{x} \boldsymbol{u} \\
& +\left(\frac{\mu(\boldsymbol{v}-\boldsymbol{u})^{2}}{2 k_{\mathrm{B}} T}-\frac{3}{2}\right)(\boldsymbol{v}-\boldsymbol{u}) \cdot \frac{\nabla_{x} T}{T}, \\
\left(\boldsymbol{V}^{*} \cdot \nabla\right) \varphi= & (1-\Pi)\left((\boldsymbol{v}-\boldsymbol{u}) \cdot \nabla_{x} \varphi\right) .
\end{aligned}
$$

The structure of the invariance equation (5.26) suggests the way of inverting the enhanced operator $\Lambda^{*}-\left(\boldsymbol{V}^{*} \cdot \nabla\right)$ :

- Step 1: Discard the enhanced free flight operator. The resulting local in space linear integral equation, $\Lambda^{*}[\varphi]=D$, is similar to the ChapmanEnskog equation (5.25) and has a unique solution by Fredholm alternative:

$$
\varphi_{\text {loc }}(\boldsymbol{x})=\left(\Lambda_{\boldsymbol{x}}^{*}\right)^{-1}[D(\boldsymbol{x})] .
$$

Here we have explicitly indicated the space variables in order to stress the fact of locality. (For a given $\boldsymbol{x}$, both $D(\boldsymbol{x})$ and $\varphi_{\text {loc }}(\boldsymbol{x})$ are functions of $\boldsymbol{x}$ and $\boldsymbol{v}$ and $\Lambda_{\boldsymbol{x}}$ is an integral in $\boldsymbol{v}$ operator.)

- Step 2: Fourier-transform the local solution,

$$
\hat{\varphi}_{\mathrm{loc}}(\boldsymbol{k})=\int e^{-i \boldsymbol{k} \cdot \boldsymbol{x}} \varphi_{\mathrm{loc}}(\boldsymbol{x}) \mathrm{d} \boldsymbol{x} .
$$

- Step 3: Replace the Fourier-transformed enhanced free flight operator with its main symbol and solve the linear integral equation,

$$
\left[\Lambda_{\boldsymbol{x}}^{*}+i\left(\boldsymbol{V}_{\boldsymbol{x}}^{*} \cdot \boldsymbol{k}\right)\right][\hat{\varphi}(\boldsymbol{x}, \boldsymbol{k})]=\hat{D}(\boldsymbol{x}, \boldsymbol{k}),
$$

where

$$
\hat{D}(\boldsymbol{x}, \boldsymbol{k})=\Lambda_{\boldsymbol{x}}^{*}\left[\hat{\varphi}_{\mathrm{loc}}(\boldsymbol{k})\right]
$$

- Step 4: Back-transform the result

$$
\varphi=(2 \pi)^{-3} \int e^{i \boldsymbol{k} \cdot \boldsymbol{x}} \hat{\varphi}(\boldsymbol{x}, \boldsymbol{k}) \mathrm{d} \boldsymbol{k} ;
$$

the resulting $\varphi$ is a function of $\boldsymbol{x}$ and $\boldsymbol{v}$.

Several comments are in order here. The above approach to solving the invariance equation is the realization of the Fourier integral operator and parametrix expansion techniques [130, 141]. The equation appearing in Step 3 is in fact the first term of the parametrix expansion. At each step of the algorithm, one needs to solve linear integral equations of the type familiar from the standard literature on the Boltzmann equation. Solutions at each step are unique by the Fredholm alternative. In practice, a good approximation for such linear integral equations is achieved by a projection on a finite-dimensional basis. Even with these approximations, evaluation of the correction to the local Maxwellian remains rather involved. Nevertheless, several results in limiting cases were obtained and are reviewed below.

For the unidirectional flow near the global equilibrium $\left(n=n_{0}, \boldsymbol{u}=0, T=T_{0}\right)$ for Maxwell's molecules, the iteration gives the following expressions for the $x x$ 


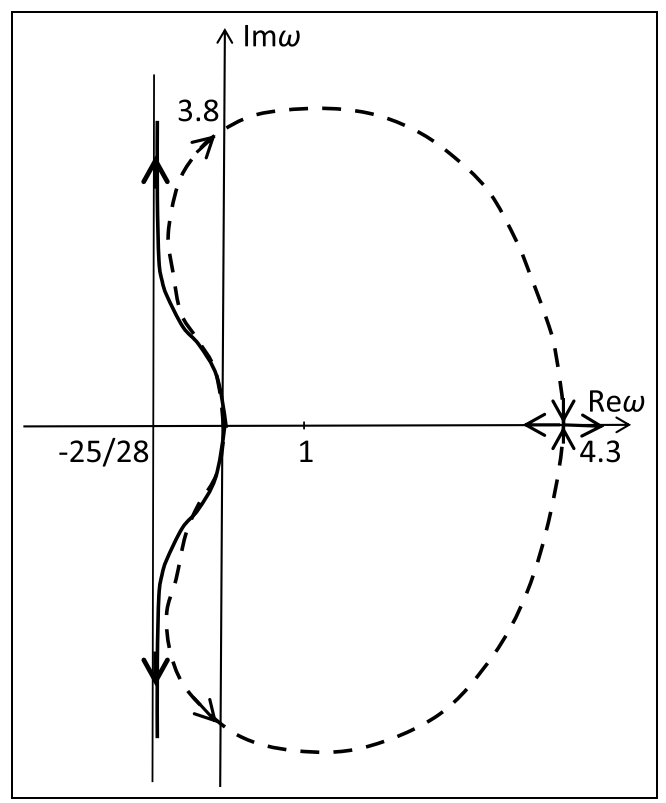

Figure 7. Acoustic dispersion curves for the frequency-response nonlocal approximation (5.33) (solid line) and for the Burnett approximation of the Chapman-Enskog expansion [9] (dashed line). Arrows indicate the direction of increase of $k^{2}$.

component of the stress tensor $\sigma$ and the $x$ component of the heat flux $q$ for onedimensional solutions (in the corresponding dimensionless variables (1.4)):

$$
\begin{aligned}
& \sigma=-\frac{2}{3} n_{0} T_{0}\left(1-\frac{2}{5} \partial_{x}^{2}\right)^{-1}\left(2 \partial_{x} u-3 \partial_{x}^{2} T\right), \\
& q=-\frac{5}{4} n_{0} T_{0}^{3 / 2}\left(1-\frac{2}{5} \partial_{x}^{2}\right)^{-1}\left(3 \partial_{x} T-\frac{8}{5} \partial_{x}^{2} u\right)
\end{aligned}
$$

The corresponding dispersion curves are presented in Figure 7 , where the saturation effect is obvious.

Already at the first iteration, the nonlinear terms are strongly coupled with the nonlocality in expressions for $\sigma$ in $q$ (see $[53,60]$ ). Viscosity tends to positive infinity for the high speed of compression (large negative div $\boldsymbol{u}$ ). In other words, the flow becomes "infinitely viscous" when $\partial_{x} u$ approaches the critical negative value $-u_{x}^{*}$. This infinite viscosity threshold prevents a transfer of the flow into the nonphysical region of negative viscosity if $\partial_{x} u<-u_{x}^{*}$ because of the "infinitely strong damping" at $-u_{x}^{*}$.

The large positive values of $\partial_{x} u$ means that the gas diverges rapidly and the flow becomes nonviscid because the particles retard to exchange their momentum. On contrary, its negative values (near $-u_{x}^{*}$ ) describe an extremely strong compression of the flow, which results in a "solid jet" limit with an infinite viscosity [55]. 
As an example, we present the result of the above microlocal analysis for the part of the stress tensor $\sigma$ which does not vanish when $T$ and $n$ are fixed:

$$
\begin{aligned}
\sigma(x) & =-\frac{1}{6 \pi} n(x) \int_{-\infty}^{+\infty} \mathrm{d} y \int_{-\infty}^{+\infty} \mathrm{d} k \exp (i k(x-y)) \frac{2}{3} \partial_{y} u(y) \\
& \times\left[\left(n(x) \lambda_{3}+\frac{11}{9} \partial_{x} u(x)\right)\left(n(x) \lambda_{4}+\frac{27}{4} \partial_{x} u(x)\right)+\frac{k^{2} v_{T}^{2}(x)}{9}\right]^{-1} \\
& \times\left[\left(n(x) \lambda_{3}+\frac{11}{9} \partial_{x} u(x)\right)\left(n(x) \lambda_{4}+\frac{27}{4} \partial_{x} u(x)\right)\right. \\
& +\frac{4}{9}\left(n(y) \lambda_{4}+\frac{27}{4} \partial_{y} u(y)\right) v_{T}^{-2}(x)(u(x)-u(y))^{2} \partial_{x} u(x) \\
& \left.-\frac{2}{3} i k(u(x)-u(y)) \partial_{x} u(x)\right]\left(n(y) \lambda_{3}+\frac{11}{9} \partial_{y} u(y)\right)^{-1} \\
& +O\left(\partial_{x} \ln T(x), \partial_{x} \ln n(x)\right)
\end{aligned}
$$

The answer in this form does not depend on the detailed collision model. Only the general properties like conservation laws, $H$-theorem and Fredholm alternative for the linearized collision integral are used. All the specific information about the collision model is collected in the positive numbers $\lambda_{3,4}$. They are represented by quadratures in 53,60. The "residual" terms describe the part of the stress tensor governed by the temperature and density gradients.

The simplest local approximation to this singularity in $\sigma$ has the form

$$
\sigma=-\mu_{0}(T) n\left(1+\frac{\partial_{x} u}{u_{x}^{*}}\right)^{-1} \partial_{x} u .
$$

For the viscosity factor $R$ (5.7) this approximation gives (compare to (5.13) and Figure 6).

$$
R=\frac{\text { const }}{1+\partial_{x} u / u_{x}^{*}}
$$

The approximations with singularities similar to (5.35) with $u_{x}^{*}=3 / 7$ have been also obtained by the partial summation of the Chapman-Enskog series [49, 50].

As we can see, the invariance correction results in a strong coupling between nonlocality and nonlinearity, and is far from the conventional Navier-Stokes and Euler equation or other truncations of the Chapman-Enskog series. Results of the microlocal correction to the local Maxwellian are quite similar to the summation of the selected main terms of the Chapman-Enskog expansion. In general, the question about the hydrodynamic invariant manifolds for the Boltzmann equation remains less studied so far because the coupling between the nonlinearity and the nonlocality brings about new challenges in calculations and proofs. There is hardly a reason to expect that the invariant manifolds for the genuine Boltzmann equation will have a nice analytic form similar to the exactly solvable reduction problem for the linearized Grad equations. Nevertheless, some effects persist: the saturation of dissipation for high frequencies and the nonlocal character of the hydrodynamic equations. 


\section{The PRoJection PROBlem AND THE ENTROPy EQUATION}

The exact invariant manifolds inherit many properties of the original systems: conservation laws, dissipation inequalities (entropy growth), and hyperbolicity of the exactly reduced system follow from these properties of the original system. The reason for this inheritance is simple: the vector field of the original system is tangent to the invariant manifold and if $M(t)$ is a solution to the exact hydrodynamic equations then, after the lifting operation, $f_{M(t)}$ is a solution to the original kinetic equation.

In real-world applications, we very rarely meet the exact reduction from kinetics to hydrodynamics, and we should work with the approximate invariant manifolds. If $\boldsymbol{f}_{M}$ is not an exact invariant manifold, then a special projection problem arises [51, 59, 120]: How should we define the projection of the vector field on the manifold $\boldsymbol{f}_{M}$ in order to preserve the most important properties, the conservation laws (first law of thermodynamics) and the positivity of entropy production (second law of thermodynamics). For hydrodynamics, the existence of the "natural" moment projection $m$ (5.16) masks the problem.

The problem of dissipativity preservation attracts much attention in the theory of shock waves. For strong shocks it is necessary to use kinetic representation; for rarefied gases the Boltzmann kinetic equation gives the framework for studying the structure of strong shocks [26]. One of the common heuristic ways to use the Boltzmann equation far from local equilibrium consists of three steps:

(1) construction of a specific ansatz for the distribution function for a given physical problem;

(2) projection of the Boltzmann equation on the ansatz;

(3) estimation and correction of the ansatz (optional).

The first and, at the same time, the most successful ansatz for the distribution function in the shock layer was invented in the middle of the twentieth century. It is the bimodal Tamm-Mott-Smith approximation (see, for example, the book [26]):

$$
f(\boldsymbol{v}, \boldsymbol{x})=f_{\mathrm{TMS}}(\boldsymbol{v}, z)=a_{-}(z) f_{-}(\boldsymbol{v})+a_{+}(z) f_{+}(\boldsymbol{v}),
$$

where $z$ is the space coordinate in the direction of the shock wave motion, $f_{ \pm}(\boldsymbol{v})$ are the downstream and the upstream Maxwellian distributions, respectively. The macroscopic variables for the Tamm-Mott-Smith approximation are the coefficients $a_{ \pm}(z)$, the lifting operation is given by (6.1) but it remains unclear how to project the Boltzmann equation onto the linear manifold (6.1) and create the macroscopic equation.

To respect the second law of thermodynamics and provide positivity of entropy production, Lampis 103. used the entropy density $s$ as a new variable. The entropy density is defined as a functional of $f(\boldsymbol{v}), s(\boldsymbol{x})=-\int f(\boldsymbol{x}, \boldsymbol{v}) \ln f(\boldsymbol{x}, \boldsymbol{v}) \mathrm{d}^{3} \boldsymbol{v}$. For each distribution $f$ the time derivative of $s$ is defined by the Boltzmann equation and the chain rule,

$$
\partial_{t} s=-\int \ln f \partial_{t} f \mathrm{~d}^{3} \boldsymbol{v}=\text { entropy flux }+ \text { entropy production. }
$$

The distribution $f$ in (6.2) is defined by the Tamm-Mott-Smith approximation:

(1) calculate the density $n$ and entropy density $s$ on the Tamm-Mott-Smith approximation (6.1) as functions of $a_{ \pm}, n=n\left(a_{+}, a_{-}\right), s=s\left(a_{+}, a_{-}\right)$;

(2) find the inverse transformation $a_{ \pm}(n, s)$; 
(3) the lifting operation in the variables $n$ and $s$ is

$$
f_{(n, s)}(\boldsymbol{v})=a_{-}(n, s) f_{-}(\boldsymbol{v})+a_{+}(n, s) f_{+}(\boldsymbol{v}) .
$$

This combination of the natural projection (6.2) and the Tamm-Mott-Smith lifting operation provides the approximate equations on the Tamm-Mott-Smith manifold with positive entropy production. Several other projections have been tested computationally [80. All of them violate second law of thermodynamics because for some initial conditions the entropy production for them becomes negative at some points. Indeed, introduction of the entropy density as an independent variable with the natural projection of the kinetic equation on this variable seems to be an attractive and universal way to satisfy the second law of thermodynamics on smooth solutions, but near the equilibria this change of variables becomes singular.

Another universal solution works near equilibria (and local equilibria). The advection operator does not change entropy. Let us consider a linear approximation to a space-uniform kinetic equation near equilibrium $f^{*}(\boldsymbol{v}): \partial_{t} \delta f=K f$. The second differential of entropy generates a positive quadratic form

$$
\langle\varphi, \psi\rangle_{f^{*}}=-\left(D^{2} S\right)(\varphi, \psi)=\int \frac{\varphi \psi}{f^{*}} \mathrm{~d}^{3} \boldsymbol{v}
$$

The quadratic approximation to the entropy production is nonnegative:

$$
-\langle\varphi, K \varphi\rangle_{f^{*}} \geq 0
$$

Let $T$ be a closed linear subspace in the space of distributions. There is a unique projector $P_{T}$ onto this subspace which does not violate the positivity of entropy production for any bounded operator $K$ with property (6.4): if $-\left\langle P_{T} \varphi, P_{T} K P_{T} \varphi\right\rangle_{f^{*}} \geq 0$ for all $\varphi, \psi$ and all bounded $K$ with property (6.4), then $P_{T}$ is an orthogonal projector with respect to the entropic inner product (6.3) [58,59]. This projector acts on functions of $\boldsymbol{v}$. For a local equilibrium $f^{*}(\boldsymbol{x}, \boldsymbol{v})$ the projector is constructed for each $\boldsymbol{x}$ and acts on functions $\varphi(\boldsymbol{x}, \boldsymbol{v})$ pointwise at each point $\boldsymbol{x}$. Liu and Yu [109] also used this projector in a vicinity of local equilibria for the micro-macro decomposition in the analysis of the shock profiles and for the study nonlinear stability of the global Maxwellian states [110. Robertson studied the projection onto manifolds constructed by the conditional maximization of the entropy and the micro-macro decomposition in the vicinity of such manifolds 122 . He obtained the orthogonal projectors with respect to the entropic inner product and called this result "the equation of motion for the generalized canonical density operator".

The general case can be considered as a "coupling" of the above two examples: the introduction of the entropy density as a new variable, and the orthogonal projector with respect to entropic inner product. Let us consider all smooth vector fields with nonnegative entropy production. The projector which preserves the nonnegativity of the entropy production for all such fields turns out to be unique. This is the so-called thermodynamic projector [51,58,60]. Let us describe this projector $P$ for a given state $f$, closed subspace $T_{f}=\mathrm{imP}_{\mathrm{T}}$, and the differential $(D S)_{f}$ of the entropy $S$ at $f$. For each state $f$ we use the entropic inner product (6.3) at $f^{*}=f$. There exists a unique vector $g(f)$ such that $\langle g, \varphi\rangle_{f}=(D S)_{f}(\varphi)$ for all $\varphi$. This is nothing but the Riesz representation of the linear functional $D_{x} S$ with respect to the entropic scalar product. If $g \neq 0$, then the thermodynamic projector 


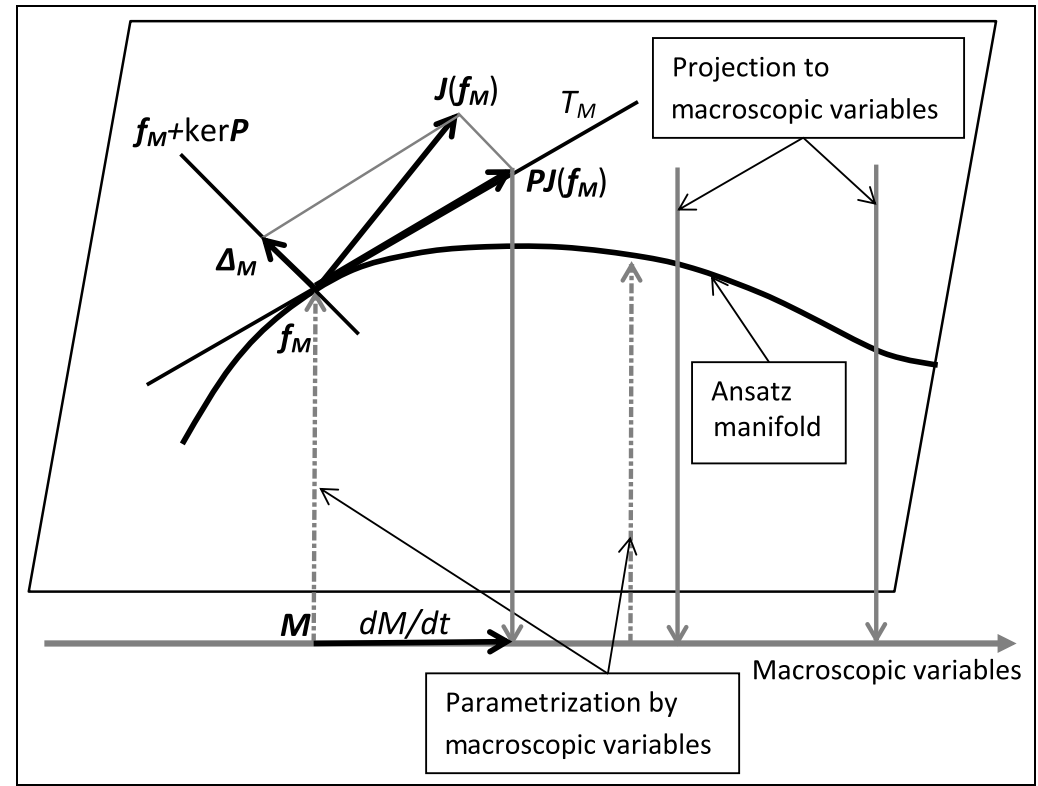

FIGURE 8. The main geometrical structures of model reduction with an approximate invariant manifold (the ansatz manifold): $J(f)$ is the vector field of the system under consideration, $\partial_{t} f=$ $J(f)$, the lifting map $M \mapsto \boldsymbol{f}_{M}$ maps a macroscopic field $M$ into the corresponding point $\boldsymbol{f}_{M}$ on the ansatz manifold, $T_{M}$ is the tangent space to the ansatz manifold at point $\boldsymbol{f}_{M}, P$ is the thermodynamic projector onto $T_{M}$ at point $\boldsymbol{f}_{M}, P J\left(\boldsymbol{f}_{M}\right)$ is the projection of the vector $J\left(\boldsymbol{f}_{M}\right)$ onto tangent space $T_{M}$, the vector field $\mathrm{d} M / \mathrm{d} t$ describes the induced dynamics on the space of macroscopic variables, $\Delta_{M}=(1-P) J\left(\boldsymbol{f}_{M}\right)$ is the defect of invariance, the affine subspace $\boldsymbol{f}_{M}+\operatorname{ker} P$ is the plain of fast motions, and $\Delta_{M} \in \operatorname{ker} P$. The invariance equation is $\Delta_{M}=0$.

of the vector field $J$ is

$$
P_{T}(J)=P^{\perp}(J)+\frac{g^{\|}}{\left\langle g^{\|} \mid g^{\|}\right\rangle_{f}}\left\langle g^{\perp} \mid J\right\rangle_{f},
$$

where $P_{T}^{\perp}$ is the orthogonal projector onto $T_{f}$ with respect to the entropic scalar product and the vector $g$ is split onto tangent and orthogonal components:

$$
g=g^{\|}+g^{\perp} ; g^{\|}=P^{\perp} g ; g^{\perp}=\left(1-P^{\perp}\right) g .
$$

This projector is defined if $g^{\|} \neq 0$. If $g^{\|}=0$ (the equilibrium point), then $J=0$ and $P(J)=P^{\perp}(J)=0$.

The selection of the projector in the form (6.5) guaranties preservation of entropy production. The thermodynamic projector can be applied for the projection of the kinetic equation onto the tangent space to the approximate invariant manifold if the differential of the entropy does not annihilate the tangent space to this manifold. (Compare to the relative entropy approach in [127.) 
Modification of the projector changes also the a simplistic picture of the separation of motions (Figure 1). The modified version is presented in Figure 8, The main differences are as follows.

- The projection of the vector field $J$ on the macroscopic variables $M$ goes in two steps:

$$
J\left(\boldsymbol{f}_{M}\right) \mapsto P J\left(\boldsymbol{f}_{M}\right) \mapsto m\left(P J\left(\boldsymbol{f}_{M}\right)\right) ;
$$

the first operation $J\left(\boldsymbol{f}_{M}\right) \mapsto P J\left(\boldsymbol{f}_{M}\right)$ projects $J$ onto the tangent plane $T_{M}$ to the ansatz manifold at point $\boldsymbol{f}_{M}$, and the second is the standard projection onto macroscopic variables $m$. Therefore, the macroscopic equations are $\partial_{t} M=m\left(P J\left(\boldsymbol{f}_{M}\right)\right)$ instead of (2.3).

- The plane of fast motion is now $\boldsymbol{f}_{M}+\operatorname{ker} P$ instead of $\boldsymbol{f}_{M}+\operatorname{ker} m$ from Figure 1.

- The entropy maximizer on $\boldsymbol{f}_{M}+\operatorname{ker} P$ is $\boldsymbol{f}_{M}$, exactly as the local Maxwellians $\boldsymbol{f}_{M}^{\mathrm{LM}}$ are the entropy maximizers on $\boldsymbol{f}_{M}^{\mathrm{LM}}+\operatorname{ker} m$. Thus, the entropic projector allows us to represent an ansatz manifold as a collection of the conditional entropy maximizers.

For details of the thermodynamic projector construction, we refer to [59, 60]. Some examples with construction of the thermodynamic projector with preservation of linear conservation laws are presented in [48.

Another possible modification is a modification of the entropy functional. Recently, Grmela [70,71] proposed to modify the entropy functional after each step of the Chapman-Enskog expansion in order to transform the approximate invariant manifold into the manifold of the conditional entropy maximizers. This idea is very similar to the thermodynamic projector in the following sense: any point $\varphi$ on the approximate invariant manifold is the conditional entropy maximum on the linear manifold $\varphi+\operatorname{ker} P_{T}$, where $T=T_{\varphi}$ is the tangent subspace to the manifold at point $\varphi$. Both modifications represent the approximate invariant manifold as a set of conditional maximizers of the entropy.

\section{Conclusion}

It is useful to solve the invariance equation. This is a particular case of the Newton's famous sentence: "It is useful to solve differential equations" ("Data aquatione quotcunque fluentes quantitce involvente fluxiones invenire et vice versa", translation published by V. I. Arnold [5]). The importance of the invariance equation has been recognized in mechanics by Lyapunov in his thesis (1892) [11]. The problem of persistence and bifurcations of invariant manifolds under perturbations is one of the most seminal problems in dynamics [2, 4, 79, 97, 139].

Several approaches to computation of invariant manifolds have been developed: Lyapunov series [111], methods of geometric singular perturbation theory [36, 37, 83, and various power series expansions [8, 24, 35]. The graph transformation approach was invented by Hadamard in 1901 [74] and developed further by many authors [41,73, 79, 98. The Newton-type direct iteration methods in various forms [51 53, 102, 124 proved their efficiency for model reduction and calculation of slow manifolds in kinetics. There is also a series of numerical methods based on the analysis of motion of an embedded manifold along the trajectories with subtraction of the motion of the manifold "parallel to themselves" [40,60,62, 119. 
The Chapman-Enskog method [24,35] was proposed in 1916. This method aims to construct the invariant manifold for the Boltzmann equation in the form of a series in powers of a small parameter, the Knudsen number Kn. This invariant manifold is parametrized by the hydrodynamic fields (density, velocity, temperature). The zeroth-order term of this series is the corresponding local equilibrium. This form of the solution (the power series and the local equilibrium zeroth term) is, at the same time, a selection rule that is necessary to choose the hydrodynamic (or Chapman-Enskog) solution of the invariance equation.

If we truncate the Chapman-Enskog series at the zeroth term, then we get the Euler hydrodynamic equations: the first term gives the Navier-Stokes hydrodynamics but already the next term (Burnett) is singular and gives negative viscosity for large divergence of the flow and instability of short waves. Nevertheless, if we apply, for example, the Newton-Kantorovich method [53,60,62, then all these singularities vanish (Section 5.2).

The Chapman-Enskog expansion appears as the Taylor series for the solution of the invariance equation. Truncation of this series may approximate the hydrodynamic invariant manifold in some limit cases, such as the long wave limit or a vicinity of the global equilibrium. Of course, the results of the invariant manifold approach should coincide with the proven hydrodynamic limits of the Boltzmann kinetics [6, 46, 108, 126, 127] "at the end of relaxation".

In general, there is no reason to hope that a few first terms of the Taylor series give an appropriate global approximation of solutions of the invariance equation (4.5). This is clearly demonstrated by the exact solutions (Sections 3 and 4).

The invariant manifold idea was present implicitly in the original Enskog and Chapman works and in most subsequent publications and textbooks. An explicit formulation of the invariant manifold program for the derivation of fluid mechanics and hydrodynamic limits from the Boltzmann equation was published by McKean [114] (see Figure 2 in Section 2.1). At the same time, McKean noticed that the problem of the invariant manifold for kinetic equations does not include the small parameter because, by the rescaling of the space dependence of the initial conditions, we can remove the coefficient in front of the collision integral: there is no difference between the Boltzmann equations with different $K n$. Now we know that the formal "small" parameter is necessary for the selection of the hydrodynamic branch of the solutions of the invariance equation because this equation can have many more solutions. (For example, for this purpose Lyapunov used analyticity of the invariant manifold and selected the zeroth approximation in the form of the invariant subspace of the linear approximation.)

The simplest example of invariant manifold is a trajectory (invariant curve). Therefore, the method of invariant manifold may be used for the construction and analysis of the trajectories. This simple idea is useful and the method of invariant manifold was applied for solution of the following problems:

- for analysis and correction of the Tamm-Mott-Smith approximation of strong shock waves far from local equilibrium [51], with the Newton iterations for corrections;

- for analysis of reaction kinetics [18] and reaction-diffusion equations [115;

- for lifting of shock waves from the piecewise solutions of the Euler equation to the solutions of the Boltzmann equation near local equilibrium for small Kn [109; 
- for analytical approximation of the relaxation trajectories 64 (the method is tested for the space-independent Boltzmann equation with various collisional mechanisms).

The invariant manifold approach to the kinetic part of Hilbert's 6th Problem. This approach, as it concerns the limit transition from the Boltzmann kinetics to mechanics of continua, was invented by Enskog almost a century ago, in 1916 [35. From a physical perspective, it remains the main method for the construction of macroscopic dynamics from dissipative kinetic equations. Mathematicians, in general, pay less attention to this approach because usually in its formulation the solution procedure (the algorithm for the construction of the bulky and singular Chapman-Enskog series) is not separated from the problem statement (the hydrodynamic invariant manifold). Nevertheless, since the 1960s the invariant manifold statement of the problem has been clear for some researchers [53, 60, 114].

Analysis of the simple kinetic models with algebraic hydrodynamic invariant manifolds (Section 31) shows that the hydrodynamic invariant manifolds may exist globally, and the divergence of the Chapman-Enskog series does not mean the nonexistence or nonanalyticity of this manifold.

The invariance equation for the more complex Grad kinetic equations (linearized) is also obtained in an algebraic form (see Section 4.3 and 60,90 for one-dimensional and Section 4.4 and 20 for three-dimensional space). An analysis of these polynomial equations shows that the real-valued solution of the invariance equation in the $k$-space may break down for very short waves. This effect is caused by the so-called entanglement of hydrodynamic and nonhydrodynamic modes.

The linearized equation with the BGK collision model [7] includes the genuine free flight advection operator and is closer to the Boltzmann equation in the hierarchy of simplifications. For this equation, there are numerical indications that the hydrodynamic modes are separated from the nonhydrodynamic ones and the calculations show that the hydrodynamic invariant manifold may exist globally (for all values of the wave vector $k$ ) 87 .

It seems more difficult to find a nonlinear Boltzmann equation with an exactly solvable invariance equation and to summarize the Chapman-Enskog series for a nonlinear kinetic equation exactly. Instead of this, we select in each term of the series the terms of the main order in the power of the Mach number $M a$ and exactly summarize the resulting series for the simple nonlinear one-dimensional Grad system (Section [5.1, 60, 90]). This expansion gives the dependence of the viscosity on the velocity gradient (5.7), (5.11), (5.13).

The exact hydrodynamics projected from the invariant manifolds inherits many useful properties of the initial kinetics: conservation laws, dissipation inequalities, and (for the bounded lifting operators) hyperbolicity (Section 4.2). Also, the existence and uniqueness theorem may be valid in the projections if it is valid for the original kinetics. In applications, for the approximate hydrodynamic invariant manifolds, the projected equation may violate many important properties. In this case, the change of the projector operator solves some of these problems (Section 6). The construction of the thermodynamic projector guarantees the positivity of entropy production even in very rough approximations [51,59].

At the present time, Hilbert's 6th Problem is not completely solved in its kinetic part. More precisely, there are several hypotheses we can prove or refute. The Hilbert hypothesis has not been unambiguously formulated but following 
his own works in the Boltzmann kinetics we can guess that he expected to receive the Euler and Navier-Stokes equations as an ultimate hydrodynamic limit of the Boltzmann equation.

Now, the Euler limit is proven for the limit $K n, M a \rightarrow 0, M a \ll K n$, and the Navier-Stokes limit is proven for $K n, M a \rightarrow 0, M a \sim K n$. In these limits, the flux is extremely slow and the gradients are extremely small (the velocity, density, and temperature do not change significantly over a long distance). The system is close to the global equilibrium. Of course, after rescaling, these solutions restore some dynamics but this rescaling erases some physically important effects. For example, it is a simple exercise to transform an attenuation curve with saturation from Figure 3 into a parable (Navier-Stokes) or even into a horizontal straight line (no attenuation, the Euler limit) with arbitrary accuracy by the rescaling of space and time.

We can state at present that beyond this limit, the Euler and Navier-Stokes hydrodynamics do not provide the proper hydrodynamic limit of the Boltzmann equation. A solution of the Boltzmann equation relaxes to the equilibrium [29] and, on its way to equilibrium, the classical hydrodynamic limit will be achieved as an intermediate asymptotic (after the proper rescaling). This recently proven result fills an important gap in our knowledge about the Boltzmann equation but from the physics perspective this is still the limit $K n, M a \rightarrow 0$ (with the proof that this limit will be achieved on the path to equilibrium).

The invariant manifold hypothesis was formulated clearly by McKean [114] (see Section 2.1, Figure 2, and Section 3.1): the kinetic equation admits an invariant manifold parametrized by the hydrodynamic fields, and the Chapman-Enskog series are the Taylor series for this manifold. Nothing is expected to be small, and no rescaling is needed. After the publication of the McKean work (1965), this hypothesis was supported by exactly solved reduction problems, explicitly calculated algebraic forms of the invariance equation and direct numerical solutions of these equations for some cases like the linearized BGK equation.

In addition to the existence of the hydrodynamic invariant manifold some stability conditions of this manifold are needed in practice. Roughly speaking, the relaxation to this manifold should be faster than the motion along it. An example of such a condition gives the separation of the hydrodynamic and nonhydrodynamic modes for linear kinetic equations (see the examples in Sections 4 and 3). It should be stressed that the strong separation of the relaxation times (Figure 1) is impossible without a small parameter. For the $\varepsilon=1$ approach, we can expect only some dominance of the relaxation toward the hydrodynamic manifold over the relaxation along it.

The capillarity hypothesis was proposed very recently by Slemrod [134, 135. He advocated the $\varepsilon=1$ approach and studied the exact sum of the Chapman-Enskog series obtained in [57, 90. Slemrod demonstrated that in the balance of the kinetic energy (3.7) a capillarity term appears (3.11) and the saturation of dissipation can be represented as the interplay between viscosity and capillarity (Section 3.3).

On the basis of this idea and some heuristics about the relation between the moment (Grad) equations and the genuine Boltzmann equation, Slemrod suggested that the proper exact hydrodynamic equation should have the form of the Korteweg hydrodynamics 32, 99, 133, rather than of Euler or Navier-Stokes ones. 
The capillarity-like terms appear, indeed, in the energy balance for all hydrodynamic equations found as a projection of the kinetic equations onto the exact or approximate invariant hydrodynamic manifolds. In that ("wide") sense, the capillarity hypothesis is plausible. In the more narrow sense, as in the assumption about the validity of the Korteweg hydrodynamics, the capillarity hypothesis requires some efforts for reformulation. The interplay between nonlinearity and nonlocality on the hydrodynamic manifolds seems to be much more complex than in the Korteweg equations (see, for example, Section [5.2, equation (5.34), or [53,60]). For a serious consideration of this hypothesis we have to find out for which asymptotic assumption we expect it to be valid (if $\varepsilon=1$, then this question is nontrivial).

\section{In the context of the exact solution of the invariance equations, three problems become visible.}

(1) Proving the existence of the hydrodynamic invariant manifold for the linearized Boltzmann equation.

(2) Proving the existence of the analytic hydrodynamic invariant manifold for the Boltzmann equation.

(3) Matching the low-frequency, small gradient asymptotics of the invariant manifold with the high-frequency, large gradient asymptotics and proving the universality of the matched asymptotics in some limits.

The first problem seems to be not extremely difficult. For its positive solution, the linearized collision operator should be bounded and satisfy the spectral gap condition.

For the nonlinear Boltzmann equation, the existence of the analytic invariant manifold seems to be plausible, but the singularities in the first NewtonKantorovich approximation (Section 5.2) may give a hint about the possible difficulties in the highly nonlinear regions. In this first approximation, flows with very high negative divergence cannot appear in the evolution of flows with lower divergence because the viscosity tends to infinity. This "solid jet" [55] effect can be considered as a sort of phase transition.

The idea of an exact hydrodynamic invariant manifold is attractive, and the approximate solutions of the invariance equation can be useful, but the possibility of elegant asymptotic solution is very attractive too. Now we know that we do not know how to state the proper problem. Can the observable hydrodynamic regimes be considered as solutions of a simplified hydrodynamic equation? Here a new, yet nonmathematical notion appears, "the observable hydrodynamic regimes". We can speculate now, that when the analytic invariant manifold exists, then together with the low-frequency, low-gradient Chapman-Enskog asymptotics the high-frequency and high-gradient asymptotics of the hydrodynamic equations are also achievable in a constructive simple form (see examples in Sections 3.6 and 5.1). The bold hypothesis (3) means that in some asymptotic sense, only the extreme cases are important and the behavior of the invariant manifold between them may be substituted by matching asymptotics. We still do not know an exact formulation of this hypothesis and can only guess how the behaviour of the hydrodynamic solutions becomes dependent only on the extreme cases. Some hints may be found in recent works about the universal asymptotics of solutions of PDEs with small dissipation [31] (which develop the ideas of Il'in proposed in the analysis of boundary layers 82 ). 
We hope that problem (1) about the existence of hydrodynamic invariant manifolds for the linearized Boltzmann equation will be solved soon; problem (2) about the full nonlinear Boltzmann equation may be approached and solved after the first one. We expect that the answer will be positive: hydrodynamic invariant manifolds do exist under the spectral gap condition.

Once the first two problems are solved, then the entire object - the hydrodynamic invariant manifold - will be outlined. For this manifold, the various asymptotic expansions could be produced, for low frequencies and gradients, for high frequencies, and for large gradients. Matching of these expansions and analysis of the resulting equations may give material for the exploration of hypothesis (3). Some guesses about the resulting equations may be formulated now, on the basis of the known results. For example we can expect that nonlocality may be reduced to the substitution of the time derivative $\partial_{t}$ in the system of fluid dynamic equations by $(1-W \Delta) \partial_{t}$, where $\Delta$ is the Laplace operator and $W$ is a positive definite matrix (compare to Section [3.6). It seems interesting and attractive that the resulting equations may be new and, at the same time, simple and beautiful hydrodynamic equations.

From the mathematical perspective, the approach based on the invariance equation now creates more questions than answers. It changes the problem statement and the exact solutions give us some hints about the possible answers.

\section{ACKNOWLEDGMENTS}

We are grateful to M. Gromov for stimulating discussion, to M. Slemrod for inspiring comments and ideas, and to L. Saint-Raymond for useful comments. The second author gratefully acknowledges support by the European Research Council (ERC) Advanced Grant 291094-ELBM.

\section{ABOUT THE AUTHORS}

Professor Alexander N. Gorban holds a personal chair in Applied Mathematics at the University of Leicester since 2004. He worked for Russian Academy of Sciences, Siberian Branch (Krasnoyarsk, Russia), and ETH Zürich (Switzerland), was a visiting professor and research scholar at Clay Mathematics Institute (Cambridge, MA), IHES (Bures-sur-Yvette, Île de France), Courant Institute of Mathematical Sciences (New York), and Isaac Newton Institute for Mathematical Sciences (Cambridge, UK). His main research interests are dynamics of systems of physical, chemical and biological kinetics; biomathematics; data mining and model reduction problems.

Professor Ilya Karlin is faculty member at the Department of Mechanical and Process Engineering, ETH Zurich, Switzerland. He was Alexander von Humboldt Fellow at the University of Ulm (Germany), CNR Fellow at the Institute of Applied Mathematics CNR "M. Picone" (Rome, Italy), and Senior Lecturer in Multiscale Modeling at the University of Southampton (England). His main research interests include exact and nonperturbative results in kinetic theory, fluid dynamics, entropic lattice Boltzmann method, model reduction for combustion systems. 


\section{REFERENCES}

[1] Igor S. Aranson and Lorenz Kramer, The world of the complex Ginzburg-Landau equation, Rev. Modern Phys. 74 (2002), no. 1, 99-143, DOI 10.1103/RevModPhys.74.99. MR.1895097 (2003h:35254)

[2] V. I. Arnold, Proof of a theorem of A.N. Kolmogorov on the invariance of quasi-periodic motions under small perturbations of the Hamiltonian, Russian Math Surveys 18 (1963), no. $5,9-36$.

[3] V. I. Arnol'd, Small denominators and problems of stability of motion in classical and celestial mechanics, Uspehi Mat. Nauk 18 (1963), no. 6 (114), 91-192 (Russian). MR0170705 (30 \#943)

[4] V. I. Arnol'd, Bifurcations of invariant manifolds of differential equations, and normal forms of neighborhoods of elliptic curves, Funkcional. Anal. i Priložen. 10 (1976), no. 4, 1-12 (Russian). MR0431285 (55 \#4286)

[5] V. I. Arnol'd, Geometrical methods in the theory of ordinary differential equations, Grundlehren der Mathematischen Wissenschaften [Fundamental Principles of Mathematical Science], vol. 250, Springer-Verlag, New York, 1983. Translated from the Russian by Joseph Szücs; Translation edited by Mark Levi. MR695786 (84d:58023)

[6] Claude Bardos, François Golse, and David Levermore, Fluid dynamic limits of kinetic equations. I. Formal derivations, J. Statist. Phys. 63 (1991), no. 1-2, 323-344, DOI 10.1007/BF01026608. MR1115587 (92d:82079)

[7] P. L. Bhatnagar, E. P. Gross and M. Krook, A model for collision processes in gases. I. Small amplitude processes in charged and neutral one-component systems, Phys. Rev. 94 (1954), no. 3, 511-525.

[8] Wolf-Jürgen Beyn and Winfried Kleß, Numerical Taylor expansions of invariant manifolds in large dynamical systems, Numer. Math. 80 (1998), no. 1, 1-38, DOI 10.1007/s002110050357. MR1642582 (99e:34054)

[9] A. V. Bobylëv, On the Chapman-Enskog and Grad methods for solving the Boltzmann equation, Dokl. Akad. Nauk SSSR 262 (1982), no. 1, 71-75 (Russian). MR647994 (83g:82034)

[10] A. V. Bobylëv, Exact solutions of the nonlinear Boltzmann equation and the theory of relaxation of a Maxwell gas, Teoret. Mat. Fiz. 60 (1984), no. 2, 280-310 (Russian, with English summary). MR762269 (86d:82019)

[11] A. V. Bobylëv, Quasistationary hydrodynamics for the Boltzmann equation, J. Statist. Phys. 80 (1995), no. 5-6, 1063-1083, DOI 10.1007/BF02179864. MR1349775 (96k:82058)

[12] T. Bodineau, I. Gallagher and L. Saint-Raymond, The Brownian motion as the limit of a deterministic system of hard-spheres, Preprint arXiv:1305.3397, 2013.

[13] N. N. Bogoliubov, Problems of a dynamical theory in statistical physics, Studies in Statistical Mechanics, Vol. I, North-Holland, Amsterdam, 1962, pp. 1-118. MR0136381 (24 \#B2419)

[14] Ludwig Boltzmann, Lectures on gas theory, Translated by Stephen G. Brush, University of California Press, Berkeley, 1964. MR0158708 (28 \#1931)

[15] S. G. Brush, The kind of motion we call heat: A history of the kinetic theory of gases in the 19th century, Book 2: Statistical Physics and Irreversible Processes, North Holland, Amsterdam The Netherlands, 1976.

[16] J. W. Cahn, Free energy of a nonuniform system. II. Thermodynamic basis, J. Chem. Phys. 30 (1959), 1121-1124.

[17] J. W. Cahn and J. E. Hilliard, Free energy of a nonuniform system. I. Interfacial energy. J. Chem. Phys., 28 (1958), 258-266.

[18] Eliodoro Chiavazzo, Alexander N. Gorban, and Iliya V. Karlin, Comparison of invariant manifolds for model reduction in chemical kinetics, Commun. Comput. Phys. 2 (2007), no. 5, 964-992. MR2355633(2008i:80013)

[19] Matteo Colangeli, Iliya V. Karlin, and Martin Kröger, From hyperbolic regularization to exact hydrodynamics for linearized Grad's equations, Phys. Rev. E (3) 75 (2007), no. 5, 051204, 10, DOI 10.1103/PhysRevE.75.051204. MR2361818(2008h:82076)

[20] Matteo Colangeli, Iliya V. Karlin, and Martin Kröger, Hyperbolicity of exact hydrodynamics for three-dimensional linearized Grad's equations, Phys. Rev. E (3) 76 (2007), no. 2, 022201, 4, DOI 10.1103/PhysRevE.76.022201. MR2365539(2008h:82077) 
[21] John C. Collins, Renormalization: An introduction to renormalization, the renormalization group, and the operator-product expansion, Cambridge Monographs on Mathematical Physics, Cambridge University Press, Cambridge, 1984. MR778558 (86k:81093)

[22] P. Constantin, C. Foias, B. Nicolaenko, and R. Temam, Integral manifolds and inertial manifolds for dissipative partial differential equations, Applied Mathematical Sciences, vol. 70, Springer-Verlag, New York, 1989. MR.966192 (90a:35026)

[23] Leo Corry, David Hilbert and the axiomatization of physics (1894-1905), Arch. Hist. Exact Sci. 51 (1997), no. 2, 83-198, DOI 10.1007/BF00375141. MR1465092 (98i:01016)

[24] Sydney Chapman and T. G. Cowling, The mathematical theory of non-uniform gases. An account of the kinetic theory of viscosity, thermal conduction and diffusion in gases, Third edition, prepared in co-operation with D. Burnett, Cambridge University Press, London, 1970. MR0258399(41 \#3046)

[25] L. Q. Chen, Phase-field models for microstructure evolution, Annual Review of Materials Research, 32 (2002), no. 1, 113-140.

[26] Carlo Cercignani, The Boltzmann equation and its applications, Applied Mathematical Sciences, vol. 67, Springer-Verlag, New York, 1988. MR.1313028 (95i:82082)

[27] A. Debussche and R. Temam, Inertial manifolds and slow manifolds, Appl. Math. Lett. 4 (1991), no. 4, 73-76, DOI 10.1016/0893-9659(91)90059-5. MR1117775 (92f:58161)

[28] P. Deligne, ed. Quantum fields and strings: a course for mathematicians, American Mathematical Society, Providence, RI, 1999.

[29] L. Desvillettes and C. Villani, On the trend to global equilibrium for spatially inhomogeneous kinetic systems: the Boltzmann equation, Invent. Math. 159 (2005), no. 2, 245-316, DOI 10.1007/s00222-004-0389-9. MR2116276 (2005j:82070)

[30] R. L. Dobrushin and Brunello Tirozzi, The central limit theorem and the problem of equivalence of ensembles, Comm. Math. Phys. 54 (1977), no. 2, 173-192. MR0445641(56 \#3977)

[31] B. Dubrovin and M. Elaeva, On the critical behavior in nonlinear evolutionary PDEs with small viscosity, Russ. J. Math. Phys. 19 (2012), no. 4, 449-460, DOI 10.1134/S106192081204005X. MR3001079

[32] J. E. Dunn and J. Serrin, On the thermomechanics of interstitial working, Arch. Rational Mech. Anal. 88 (1985), no. 2, 95-133, DOI 10.1007/BF00250907. MR775366 (86f:73051)

[33] F. J. Dyson, Divergence of perturbation theory in quantum electrodynamics, Physical Rev. (2) 85 (1952), 631-632. MR0046927 (13,805f)

[34] John Ellis, Einan Gardi, Marek Karliner, and Mark A. Samuel, Padé approximants, Borel transforms and renormalons: the Bjorken sum rule as a case study, Phys. Lett. B 366 (1996), no. 1-4, 268-275, DOI 10.1016/0370-2693(95)01326-1. MR.1371554 (96k:81174)

[35] D. Enskog, Kinetische theorie der Vorange in massig verdunnten Gasen. I Allgemeiner Teil, Almqvist and Wiksell, Uppsala, 1917.

[36] Neil Fenichel, Persistence and smoothness of invariant manifolds for flows, Indiana Univ. Math. J. 21 (1971/1972), 193-226. MR0287106 (44 \#4313)

[37] Neil Fenichel, Geometric singular perturbation theory for ordinary differential equations, J. Differential Equations 31 (1979), no. 1, 53-98, DOI 10.1016/0022-0396(79)90152-9. MR:524817 (80m:58032)

[38] R. P. Feynman, The character of physical law, MIT Press, Cambridge, MA, 1965.

[39] M. E. Fisher, The renormalization group in the theory of critical behavior, Reviews of Modern Physics 46 (1974), no. 4, 597-616.

[40] C. Foias, M. S. Jolly, I. G. Kevrekidis, G. R. Sell, and E. S. Titi, On the computation of inertial manifolds, Phys. Lett. A 131 (1988), no. 7-8, 433-436, DOI 10.1016/03759601(88)90295-2. MR972615 (89k:65154)

[41] E. Fontich, Transversal homoclinic points of a class of conservative diffeomorphisms, J. Differential Equations 87 (1990), no. 1, 1-27, DOI 10.1016/0022-0396(90)90012-E. MR.1070024 (91j:58095)

[42] N. Fröman and P. O. Fröman, JWKB Approximation, North-Holland, Amsterdam, 1965.

[43] I. Gallagher, L. Saint-Raymond, and B. Texier, From Newton to Boltzmann: hard spheres and short-range potentials, Preprint arXiv:1208.5753, 2012.

[44] Vicente Garzó and Andrés Santos, Kinetic theory of gases in shear flows. Nonlinear transport, Fundamental Theories of Physics, vol. 131, Kluwer Academic Publishers Group, Dordrecht, 2003. MR2012795(2005b:76115) 
[45] J. Gnedenko, Zum sechsten Hilbertschen Problem, In Die Hilbertsche Probleme (ed. by P. Alexandrov), Ostwalds Klassiker der exakten Wissenschaften 252, Leipzig 1979, 144-147.

[46] François Golse and Laure Saint-Raymond, The Navier-Stokes limit of the Boltzmann equation for bounded collision kernels, Invent. Math. 155 (2004), no. 1, 81-161, DOI 10.1007/s00222-003-0316-5. MR2025302 (2005f:76003)

[47] A. N. Gorban, Order-disorder separation: geometric revision, Physica A 374 (2007), no. 1, 85-102.

[48] A. N. Gorban, P. A. Gorban, and I. V. Karlin, Legendre integrators, post-processing and quasiequilibrium, J. Non-Newtonian Fluid Mech. 120 (2004), 149-167

[49] A. N. Gorban and I. V. Karlin, Structure and approximations of the Chapman-Enskog expansion, Sov. Phys. JETP 73 (1991), 637-641.

[50] Alexander N. Gorban and Iliya V. Karlin, Structure and approximations of the ChapmanEnskog expansion for the linearized Grad equations, Transport Theory Statist. Phys. 21 (1992), no. 1-2, 101-117, DOI 10.1080/00411459208203524. MR.1149364 (92m:82117)

[51] Alexander N. Gorban and Iliya V. Karlin, Thermodynamic parameterization, Phys. A 190 (1992), no. 3-4, 393-404, DOI 10.1016/0378-4371(92)90044-Q. MR1196414 (93m:82040)

[52] Alexander N. Gorban and Iliya V. Karlin, Letter to the editor: nonarbitrary regularization of acoustic spectra, Transport Theory Statist. Phys. 22 (1993), no. 1, 121-124, DOI 10.1080/00411459308203534. MR.1198882 (94c:82064)

[53] Alexander N. Gorban and Iliya V. Karlin, Method of invariant manifolds and regularization of acoustic spectra, Transport Theory Statist. Phys. 23 (1994), no. 5, 559-632, DOI 10.1080/00411459408204345. MR.1272676 (95c:82037)

[54] A. N. Gorban and I. V. Karlin, General approach to constructing models of the Boltzmann equation, Physica A 206 (1994), 401-420.

[55] A. N. Gorban and I. V. Karlin, On "solid liquid" limit of hydrodynamic equations, Transport Theory and Stat. Phys. 24 (1995), no. 9, 1419-1421.

[56] A. N. Gorban and I. V. Karlin, Scattering rates versus moments: Alternative Grad equations, Phys. Rev. E 54 (1996), R3109-R3112.

[57] A. N. Gorban and I. V. Karlin, Short-wave limit of hydrodynamics: a soluble example, Phys. Rev. Lett. 77 (1996), 282-285.

[58] A. N. Gorban and I. V. Karlin, Method of invariant manifold for chemical kinetics, Chem. Eng. Sci., 58 (2003), 4751-4768.

[59] A. N. Gorban and I. V. Karlin, Uniqueness of thermodynamic projector and kinetic basis of molecular individualism, Physica A 336 (2004), no. 3-4, 391-432.

[60] A. N. Gorban and I. V. Karlin, Invariant Manifolds for Physical and Chemical Kinetics, Lecture Notes in Physics, vol. 660, Springer-Verlag, Berlin, 2005. MR 2163015 (2007f:80008)

[61] Alexander N. Gorban and Iliya V. Karlin, Quasi-equilibrium closure hierarchies for the Boltzmann equation, Phys. A 360 (2006), no. 2, 325-364, DOI 10.1016/j.physa.2005.07.016. MR2186263 (2007a:82064)

[62] Alexander N. Gorban, Iliya V. Karlin, and Andrei Yu. Zinovyev, Constructive methods of invariant manifolds for kinetic problems, Phys. Rep. 396 (2004), no. 4-6, 197-403, DOI 10.1016/j.physrep.2004.03.006. MR2061613(2005a:82090)

[63] Alexander N. Gorban, Iliya V. Karlin, and Vladimir B. Zmievskii, Two-step approximation of space-independent relaxation, Transport Theory Statist. Phys. 28 (1999), no. 3, 271-296, DOI 10.1080/00411459908206037. MR.1686730(2000a:82060)

[64] A. N. Gorban, I. V. Karlin, V. B. Zmievskii, and T. F. Nonnenmacher, Relaxational trajectories: global approximations, Physica A 231 (1996), 648-672.

[65] A.N. Gorban and O. Radulescu, Dynamic and static limitation in multiscale reaction networks, revisited, Advances in Chemical Engineering 34 (2008), 103-173.

[66] A. N. Gorban and G. S. Yablonsky, Extended detailed balance for systems with irreversible reactions, Chemical Engineering Science 66 (2011), 5388-5399.

[67] Hermann Grabert, Projection Operator Techniques in Nonequilibrium Statistical Mechanics, Springer Tracts in Modern Physics, vol. 95, Springer-Verlag, Berlin, 1982. MR670593 (84k:82001)

[68] Harold Grad, On the kinetic theory of rarefied gases, Comm. Pure Appl. Math. 2 (1949), 331-407. MR0033674 (11,473a) 
[69] Harold Grad, Principles of the kinetic theory of gases, Handbuch der Physik (S. Flügge, editor), Bd. 12, Thermodynamik der Gase, Springer-Verlag, Berlin, 1958, pp. 205-294. MR.0135535 (24 \#B1583)

[70] M. Grmela, Multiscale equilibrium and nonequilibrium thermodynamics in chemical engineering, Advances in Chemical Engineering 39 (2010), 75-129.

[71] Miroslav Grmela, Role of thermodynamics in multiscale physics, Comput. Math. Appl. 65 (2013), no. 10, 1457-1470, DOI 10.1016/j.camwa.2012.11.019. MR 3061716

[72] Misha Gromov, Spaces and questions, Geom. Funct. Anal. Special Volume (2000), 118161. GAFA 2000 (Tel Aviv, 1999). MR1826251(2002e:53056)

[73] John Guckenheimer and Alexander Vladimirsky, A fast method for approximating invariant manifolds, SIAM J. Appl. Dyn. Syst. 3 (2004), no. 3, 232-260, DOI 10.1137/030600179. MR 2114735(2005h:37064)

[74] J. Hadamard, Sur l'iteration et les solutions asymptotiques des equations diffrentielles, Bull. Soc. Math. France 29 (1901), 224-228.

[75] E. H. Hauge, Exact and Chapman-Enskog solutions of the Boltzmann equation for the Lorentz model, Phys. Fluids 13 (1970), 1201-1208. MR0278672 (43 \#4402)

[76] David Hilbert, Mathematical problems, Bull. Amer. Math. Soc. 8 (1902), no. 10, 437-479, DOI 10.1090/S0002-9904-1902-00923-3. MR1557926

[77] David Hilbert, Begründung der kinetischen Gastheorie, Math. Ann. 72 (1912), no. 4, 562577, DOI 10.1007/BF01456676 (German). MR1511713

[78] J. O. Hirschfelder, C. F. Curtiss, and R. B. Bird, Molecular theory of gases and liquids, J. Wiley, New York, 1954.

[79] M. W. Hirsch, C. C. Pugh, and M. Shub, Invariant manifolds, Lecture Notes in Mathematics, Vol. 583, Springer-Verlag, Berlin, 1977. MR0501173 (58 \#18595)

[80] I. Hosokawa and S. Inage, Local entropy balance through the shock wave, J. Phys. Soc. Japan 55 (1986), no. 10, 3402-3409.

[81] Tadanori Hyouguchi, Ryohei Seto, Masahito Ueda, and Satoshi Adachi, Divergence-free WKB theory, Ann. Physics 312 (2004), no. 1, 177-267, DOI 10.1016/j.aop.2004.01.005. MR $2067810(2005 \mathrm{c}: 81064)$

[82] A. M. Il'in, Matching of asymptotic expansions of solutions of boundary value problems, Translations of Mathematical Monographs, vol. 102, American Mathematical Society, Providence, RI, 1992. Translated from the Russian by V. Minachin [V. V. Minakhin]. MR1182791 (93g:35016)

[83] Christopher K. R. T. Jones, Geometric singular perturbation theory, Dynamical systems (Montecatini Terme, 1994), Lecture Notes in Math., vol. 1609, Springer, Berlin, 1995, pp. 44118, DOI 10.1007/BFb0095239. MR1374108(97e:34105)

[84] D. Jou, J. Casas-Vázquez, and G. Lebon, Extended Irreversible Thermodynamics, SpringerVerlag, Berlin, 1993. MR 1271780 (95a:80004)

[85] Iliya V. Karlin, Simplest nonlinear regularization, Transport Theory Statist. Phys. 21 (1992), no. 3, 291-293, DOI 10.1080/00411459208203925. MR1165529(93d:82069)

[86] Iliya V. Karlin, Exact summation of the Chapman-Enskog expansion from moment equations, J. Phys. A 33 (2000), no. 45, 8037-8046, DOI 10.1088/0305-4470/33/45/303. MR.1804291

[87] I. V. Karlin, M. Colangeli, and M. Kröger, Exact linear hydrodynamics from the Boltzmann Equation, Phys. Rev. Lett. 100 (2008), 214503.

[88] I. V. Karlin, G. Dukek, and T. F. Nonnenmacher, Invariance principle for extension of hydrodynamics: Nonlinear viscosity, Phys. Rev. E 55 (1997), no. 2, 1573-1576.

[89] I. V. Karlin, G. Dukek, and T. F. Nonnenmacher, Gradient expansions in kinetic theory of phonons, Phys. Rev. B 55 (1997), 6324-6329.

[90] Iliya V. Karlin and Alexander N. Gorban, Hydrodynamics from Grad's equations: what can we learn from exact solutions?, Ann. Phys. 11 (2002), no. 10-11, 783-833, DOI 10.1002/15213889(200211)11:10/11/783::AID-ANDP783〉3.0.CO;2-V. MR1957348(2004e:82050)

[91] I. V. Karlin, A. N. Gorban, G. Dukek, and T. F. Nonnenmacher, Dynamic correction to moment approximations, Phys. Rev. E 57 (1998), 1668-1672.

[92] Nikolaos Kazantzis, Singular PDEs and the problem of finding invariant manifolds for nonlinear dynamical systems, Phys. Lett. A 272 (2000), no. 4, 257-263, DOI 10.1016/S03759601(00)00451-5. MR1774786(2001k:35052) 
[93] N. Kazantzis and T. Good, Invariant manifolds and the calculation of the long-term asymptotic response of nonlinear processes using singular PDEs, Computers and Chemical Engineering 26 (2002), no. 7, 999-1012.

[94] Nikolaos Kazantzis, Costas Kravaris, and Lemonia Syrou, A new model reduction method for nonlinear dynamical systems, Nonlinear Dynam. 59 (2010), no. 1-2, 183-194, DOI 10.1007/s11071-009-9531-y. MR2585285 (2011a:93038)

[95] A. I. Khinchin, Mathematical Foundations of Statistical Mechanics, Dover Publications Inc., New York, 1949. Translated by G. Gamow. MR0029808 (10,666c)

[96] A. Kolmogoroff, Grundbegriffe der Wahrscheinlichkeitsrechnung, Springer-Verlag, Berlin, 1977 (German). Reprint of the 1933 original. MR0494348 (58 \#13242)

[97] A. N. Kolmogorov, On conservation of conditionally periodic motions for a small change in Hamilton's function, Dokl. Akad. Nauk SSSR (N.S.) 98 (1954), 527-530 (Russian). MR0068687 (16,924c)

[98] A. A. Kornev, On a method of "graph transformation" type for the numerical construction of invariant manifolds, Tr. Mat. Inst. Steklova 256 (2007), no. Din. Sist. i Optim., 237-251, DOI 10.1134/S0081543807010129 (Russian, with Russian summary); English transl., Proc. Steklov Inst. Math. 256 (2007), no. 1, 223-237. MR2336902(2010a:37174)

[99] D. J. Korteweg, Sur la forme que prennent les équations du mouvements des fluides si l'on tient compte des forces capillaires causées par des variations de densité, Archives Néerl. Sci. Exactes Nat. Ser II 6 (1901), 1-24.

[100] Ryogo Kubo, Statistical-mechanical theory of irreversible processes. I. General theory and simple applications to magnetic and conduction problems, J. Phys. Soc. Japan 12 (1957), 570-586. MR0098482 (20 \#4940a)

[101] A. Kurganov and P. Rosenau, Effects of a saturating dissipation in Burgers-type equations, Comm. Pure Appl. Math. 50 (1997), no. 8, 753-771, DOI 10.1002/(SICI)10970312(199708)50:8<753::AID-CPA2〉3.0.CO;2-5. MR 1454172 (98d:35194)

[102] S. H. Lam, D. A. Goussis, The CSP method for simplifying kinetics, International Journal of Chemical Kinetics 26 (1994), 461-486.

[103] M. Lampis, New approach to the Mott-Smith method for shock waves, Meccanica 12 (1977), 171-173.

[104] Oscar E. Lanford III, Time evolution of large classical systems, Dynamical Systems, Theory and Applications (Recontres, Battelle Res. Inst., Seattle, Wash., 1974), Springer, Berlin, 1975, pp. 1-111. Lecture Notes in Phys., Vol. 38. MR0479206 (57 \#18653)

[105] Peter D. Lax and C. David Levermore, The small dispersion limit of the Kortewegde Vries equation. I, Comm. Pure Appl. Math. 36 (1983), no. 3, 253-290, DOI 10.1002/cpa.3160360302. MR697466 (85g:35105a)

[106] C. David Levermore, Moment closure hierarchies for kinetic theories, J. Statist. Phys. 83 (1996), no. 5-6, 1021-1065, DOI 10.1007/BF02179552. MR1392419 (97e:82041)

[107] Ming Li and Paul Vitányi, An introduction to Kolmogorov complexity and its applications, 2nd ed., Graduate Texts in Computer Science, Springer-Verlag, New York, 1997. MR1438307 (97k:68086)

[108] Pierre-Louis Lions and Nader Masmoudi, Une approche locale de la limite incompressible, C. R. Acad. Sci. Paris Sér. I Math. 329 (1999), no. 5, 387-392, DOI 10.1016/S07644442(00)88611-5 (French, with English and French summaries). MR.1710123(2000e:76103)

[109] Tai-Ping Liu and Shih-Hsien Yu, Boltzmann equation: micro-macro decompositions and positivity of shock profiles, Comm. Math. Phys. 246 (2004), no. 1, 133-179, DOI 10.1007/s00220-003-1030-2. MR2044894 (2005f:82101)

[110] Tai-Ping Liu, Tong Yang, and Shih-Hsien Yu, Energy method for Boltzmann equation, Phys. D 188 (2004), no. 3-4, 178-192, DOI 10.1016/j.physd.2003.07.011. MR.2043729 (2005a:82091)

[111] A. M. Lyapunov, The general problem of the stability of motion, Taylor \& Francis Ltd., London, 1992. Translated from Edouard Davaux's French translation (1907) of the 1892 Russian original and edited by A. T. Fuller; With an introduction and preface by Fuller, a biography of Lyapunov by V. I. Smirnov, and a bibliography of Lyapunov's works compiled by J. F. Barrett; Lyapunov Centenary Issue; reprint of Internat. J. Control 55 (1992), no. 3 [MR1154209 (93e:01035)]; With a foreword by Ian Stewart. MR 1229075 (94c:34074)

[112] U. Maas and S. B. Pope, Simplifying chemical kinetics: intrinsic low-dimensional manifolds in composition space, Combustion and Flame, 88 (1992), 239-264. 
[113] Richard D. Mattuck, A guide to Feynman diagrams in the many-body problem, Dover Publications Inc., New York, 1992. Reprint of the second (1976) edition. MR1191398 (93i:81002)

[114] H. P. McKean Jr., A simple model of the derivation of fluid mechanics from the Boltzmann equation, Bull. Amer. Math. Soc. 75 (1969), 1-10. MR0235792 (38 \#4095)

[115] J. D. Mengers and J. M. Powers, One-dimensional slow invariant manifolds for fully coupled reaction and micro-scale diffusion, SIAM J. Appl. Dyn. Syst. 12 (2013), no. 2, 560-595, DOI 10.1137/120877118. MR3044099

[116] Tim R. Morris, The exact renormalization group and approximate solutions, Internat. J. Modern Phys. A 9 (1994), no. 14, 2411-2449, DOI 10.1142/S0217751X94000972. MR 1277033 (95i:81169)

[117] Jürgen Moser, Convergent series expansions for quasi-periodic motions, Math. Ann. 169 (1967), 136-176. MR.0208078 (34 \#7888)

[118] Clément Mouhot, Explicit coercivity estimates for the linearized Boltzmann and Landau operators, Comm. Partial Differential Equations 31 (2006), no. 7-9, 1321-1348, DOI 10.1080/03605300600635004. MR2254617(2007h:35020)

[119] J. Nafe and U. Maas, A general algorithm for improving ILDMs, Combust. Theory Modelling 6 (2002), 697-709.

[120] V. A. Palin and E. V. Radkevich, The Navier-Stokes approximation and Chapman-Enskog projection problems for kinetic equations, Tr. Semin. im. I. G. Petrovskogo 25 (2006), 184225, 326, DOI 10.1007/s10958-006-0140-8 (Russian, with Russian summary); English transl., J. Math. Sci. (N.Y.) 135 (2006), no. 1, 2721-2748. MR2271911 (2007g:35172)

[121] H. Poincaré, Les méthodes nouvelles de la mécanique céleste, Vols. 1-3. Gauthier-Villars, Paris, 1892/1893/1899.

[122] Baldwin Robertson, Equations of motion in nonequilibrium statistical mechanics, Phys. Rev. (2) 144 (1966), 151-161. MR0198898 (33 \#7051)

[123] Philip Rosenau, Extending hydrodynamics via the regularization of the Chapman-Enskog expansion, Phys. Rev. A (3) 40 (1989), no. 12, 7193-7196, DOI 10.1103/PhysRevA.40.7193. MR.1031939 (91b:82047)

[124] M. R. Roussel and S. J. Fraser, Geometry of the steady-state approximation: Perturbation and accelerated convergence methods, J. Chem. Phys. 93 (1990), 1072-1081.

[125] Laure Saint-Raymond, From the BGK model to the Navier-Stokes equations, Ann. Sci. École Norm. Sup. (4) 36 (2003), no. 2, 271-317, DOI 10.1016/S0012-9593(03)00010-7 (English, with English and French summaries). MR 1980313 (2004f:76042)

[126] Laure Saint-Raymond, Hydrodynamic limits of the Boltzmann equation, Lecture Notes in Mathematics, vol. 1971, Springer-Verlag, Berlin, 2009. MR2683475 (2012f:82079)

[127] L. Saint-Raymond, A mathematical PDE perspective on the Chapman-Enskog expansion, Bull. Amer. Math. Soc., 51 (2014), no. 2, 247-275.

[128] A. Santos, Nonlinear viscosity and velocity distribution function in a simple longitudinal flow, Phys. Rev. E 62 (2000), 6597-6607.

[129] Lee A. Segel and Marshall Slemrod, The quasi-steady-state assumption: a case study in perturbation, SIAM Rev. 31 (1989), no. 3, 446-477, DOI 10.1137/1031091. MR.1012300 (92f:92003)

[130] M. A. Shubin, Pseudodifferential Operators and Spectral Theory, 2nd ed., Springer-Verlag, Berlin, 2001. Translated from the 1978 Russian original by Stig I. Andersson. MR 1852334 (2002d:47073)

[131] M. Slemrod, Admissibility criteria for propagating phase boundaries in a van der Waals fluid, Arch. Rational Mech. Anal. 81 (1983), no. 4, 301-315, DOI 10.1007/BF00250857. MR683192 (84a:76030)

[132] M. Slemrod, Renormalization of the Chapman-Enskog expansion: isothermal fluid flow and Rosenau saturation, J. Statist. Phys. 91 (1998), no. 1-2, 285-305, DOI 10.1023/A:1023048322851. MR 1632510 (99j:76109)

[133] Marshall Slemrod, Constitutive relations for monatomic gases based on a generalized rational approximation to the sum of the Chapman-Enskog expansion, Arch. Ration. Mech. Anal. 150 (1999), no. 1, 1-22, DOI 10.1007/s002050050178. MR1738169 (2001g:76054)

[134] Marshall Slemrod, Chapman-Enskog $\Rightarrow$ viscosity-capillarity, Quart. Appl. Math. 70 (2012), no. 3, 613-624, DOI 10.1090/S0033-569X-2012-01305-1. MR2986137

[135] M. Slemrod, From Boltzmann to Euler: Hilbert's 6th problem revisited, Comput. Math. Appl. 65 (2013), no. 10, 1497-1501, DOI 10.1016/j.camwa.2012.08.016. MR 3061719 
[136] Sauro Succi, The lattice Boltzmann equation for fluid dynamics and beyond, Numerical Mathematics and Scientific Computation, The Clarendon Press Oxford University Press, New York, 2001. Oxford Science Publications. MR 1857912 (2002k:82069)

[137] Michel Talagrand, A new look at independence, Ann. Probab. 24 (1996), no. 1, 1-34, DOI 10.1214/aop/1042644705. MR 1387624 (97d:60028)

[138] Michel Talagrand, Rigorous low-temperature results for the mean field p-spins interaction model, Probab. Theory Related Fields 117 (2000), no. 3, 303-360, DOI 10.1007/s004400050009. MR1774067 (2001f:82037)

[139] Roger Temam, Infinite-dimensional dynamical systems in mechanics and physics, Applied Mathematical Sciences, vol. 68, Springer-Verlag, New York, 1988. MR953967 (89m:58056)

[140] A. N. Tihonov, Systems of differential equations containing small parameters in the derivatives, Mat. Sbornik N.S. 31(73) (1952), 575-586 (Russian). MR0055515 (14,1085d)

[141] François Trèves, Introduction to pseudodifferential and Fourier integral operators. Vol. 1, Plenum Press, New York, 1980. Pseudodifferential operators; The University Series in Mathematics. MR597144 (82i:35173)

[142] G. E. Uhlenbeck, Some notes on the relation between fluid mechanics and statistical physics, Annual Review of Fluid Mechanics, Vol. 12, Annual Reviews, Palo Alto, Calif., 1980, pp. 1-9. MR.565387(82i:82004)

[143] G. Veneziano, Construction of a crossing-symmetric, Regge-behaved amplitude for linearly rising trajectories, Nuovo Cimento A 57 (1968), 190-197.

[144] A. Voros, The return of the quartic oscillator: the complex WKB method, Ann. Inst. H. Poincaré Sect. A (N.S.) 39 (1983), no. 3, 211-338 (English, with French summary). MR729194(86m:81051)

[145] S. Weinberg, The quantum theory of fields, Cambridge University Press (1995).

[146] J. Zinn-Justin, Quantum field theory and critical phenomena, 2nd ed., International Series of Monographs on Physics, vol. 85, The Clarendon Press Oxford University Press, New York, 1993. Oxford Science Publications. MR 1227790 (95i:81001)

[147] D. N. Zubarev, Nonequilibrium statistical thermodynamics, Studies in Soviet Science. Consultants Bureau, New York, 1974, Translated from the Russian by P. J. Shepherd. MR0468659 (57 \#8491)

[148] A. K. Zvonkin and L. A. Levin, The complexity of finite objects and the basing of the concepts of information and randomness on the theory of algorithms, Uspehi Mat. Nauk 25 (1970), no. 6 (156), 85-127 (Russian). MR0307889 (46 \#7004)

Department of Mathematics, University of Leicester, Leicester, United Kingdom

E-mail address: ag1530le.ac.uk

Department of Mechanical and Process Engineering, ETH Zürich, Switzerland

E-mail address: karlin@lav.mavt.ethz.ch 